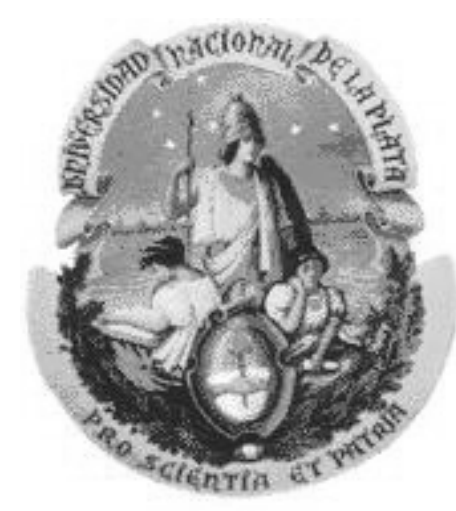

UNIVERSIDAD NACIONAL DE LA PLATA

FACULTAD DE CIENCIAS VETERINARIAS

Trabajo de Tesis realizado como requisito para optar al título de DOCTOR EN CIENCIAS VETERINARIAS

\title{
“APLICABILIDAD DE LA ULTRASONOGRAFÍA DOPPLER Y ELECTROCARDIOGRAFÍA EN LA EVALUACIÓN DE PARÁMETROS CARDIOVASCULARES MATERNO-FETALES NORMALES Y PATOLÓGICOS EN LA GESTACIÓN CANINA"
}

AUTOR: BLANCO, Paula Graciela, MV

DIRECTOR: GOBELLO, Ma. Cristina, DMV, DECAR

CODIRECTOR: ARIAS, Daniel Osvaldo, DMV

LUGAR DE TRABAJO: Servicio de Cardiología, Hospital Escuela. Facultad de Ciencias Veterinarias, Universidad Nacional de La Plata

MIEMBROS DEL JURADO:

Dr. CASTILLO, Víctor

Dra. KONCURAT, Mirta

Dr. MATTIOLI, Guillermo 
A los que amo, por hacer que la vida tenga sentido 


\section{Agradecimientos}

Haber llegado hasta acá no es sólo el resultado de mi esfuerzo individual. Tampoco es consecuencia exclusiva de acumular conocimientos y actividades académicas o de contar con la infraestructura y recursos materiales necesarios. Fueron indispensables otros muchos elementos, invisibles, impalpables, indecibles para que este proyecto sea lo que es. Todo junto en un orden revuelto, encajando dentro de un sistema ecléctico, única forma posible de concresión de esta obra, que no tuvo un resultado únicamente científico. A cada uno de los responsables de este fenómeno maravilloso, mi agradecimiento.

A mi Directora, un ejemplo de pasión por nuestra profesión. Por haberme enseñado a desempeñarme en esta tarea de manera comprometida y metódica, pero sobre todo, por hacerme ver que no hay alegría más grande que amar lo que se hace, fin último de la vida. Por haberme acompañado con abnegada paciencia, dedicación, responsabilidad y generosidad, haber confiado en mí y dedicarme tanto de su tiempo. Por transmitirme sus ganas de crecer todos los días en esta actividad y principalmente, por demostrarme con el ejemplo diario el valor de la perseverancia frente a todas las adversidades de este camino.

A mi Co-Director, por compartir todos sus conocimientos, su logro de haber creado un Servicio de Cardiología de referencia y permtirme trabajar en él. Por haberme escuchado al decir que quería dedicarme a la investigación científica y hacer lo posible para ayudarme a hacer ese sueño realidad, depositando toda su confianza en mi persona. Por ser otro modelo de gran generosidad y ser un ejemplo en muchísimos órdenes de la vida, dentro y fuera del ámbito académico. Por enseñarme un caudal tan grande de cosas con una caudal tan grande de afecto.

A mi familia, por acompañarme siempre, con tanto amor, ayer y hoy. Por enseñarme el valor del esfuerzo y del compromiso con el trabajo. Por ser un ejemplo de vida. Por estar siempre al lado mío, cada vez que los necesité. A mi mamá, que me dedicó su tesis doctoral y es el ejemplo de género que admiro.

A Ber, siempre dispuesto a ayudar, incondicional, mi guía y compañero, por tanto amor.

A mi país, por darme la posibilidad de haber llegado hasta acá. Por permitirme investigar en el lugar que me vio nacer, para su propio beneficio y el de la sociedad de la que soy parte. Por incluirme en un proyecto de Nación que aumentó en un $600 \%$ el presupuesto para Ciencia y Tecnología en los últimos años y se propone desarrollar el conocimiento científico como pilar fundamental de su capital cultural. Por hacerme parte.

Al Consejo Nacional de Investigaciones Científicas y Tecnológicas (CONICET), por permitirme dedicarme de manera exclusiva a la concreción de este proyecto, garantizándome un salario digno. A la Secretaría de Ciencia y Técnica de la Universidad Nacional de La Plata y a la Morris Animal Foundation, por los subsidios otorgados oportunamente, que me permitieron llevar a cabo esta tarea y dar a conocer parte de mi trabajo en el mundo.

A la Universidad Nacional de La Plata, que se ha encargado de mi formación desde los 3 años de edad. Por su educación laica, pública y gratuita que me ha brindado el nivel académico que hoy poseo y por ser la institución por cuyo crecimiento vale la pena seguir trabajando todos 
los días. A la Facultad de Ciencias Veterinarias de La Plata, por darme el espacio, la infraestructura y los recursos necesarios para trabajar con total libertad.

A Cacho, Mariana, Anita, Titi, Fer, Nico, Augusto, Pablo, Javi, Sil, Vane, Lupe, Daniela por haberme dado una mano cada vez que la necesité, incondicionalmente, siempre. Por hacer del Servicio de Cardiología un lugar al que siempre den ganas de llegar, por hacer que cada jornada diaria no sea sólo de trabajo, sino también de alegría, de afecto, de contención, de compañía.

A todo Métodos Complementarios de Diagnóstico y al Hospital Escuela, a los Servicios de Radiología y Endoscopía. A cada uno de sus miembros por el favor siempre hecho. A Miriam, por ser el ejemplo más grande de amor a los animales.

A mis amigos, por acompañarme y bancarme tantas veces durante tanto tiempo. A los artistas, que me invitaron a descubrir la magia del proceso cretivo y a cultivar mi propia creatividad. A todos, por las emociones y el cariño recibido.

A los criaderos De Casa Alteza y von Axmann, a sus propietarias, Anita y Vero y sus perras, con las que voy a estar eternamente agradecida. A todos los propietarios que contribuyeron con sus mascotas, dedicando parte de su tiempo a este proyecto.

A Car, Yani, Jorge, Guada, Ana, Eli, Merce, Guille, Pablo, Patricio, del Laboratorio de Fisiología Reproductiva, por toda la ayuda que me brindaron, por compartir parte de sus vidas conmigo.

Al Servicio de Cardiología del Hospital de Niños Sor María Ludovica de La Plata, a los Dres. Pedro Weisburd, Graciela Citate y Andrea Cuelli, por abrirme las puertas de su lugar de trabajo y enseñarme desinteresadamente parte de lo muchísimo que saben.

A la cátedra de Fisiología, por permitirme tener un lugar de trabajo junto a mi Directora. A Alejandro Relling por contribuir al crecimiento de los que nos iniciamos en este camino.

Finalmente a las 'nenas', para quienes va dirigido este trabajo. A todas las que colaboraron con tanta paciencia y alegría, a las que ya no están. A mis perros, mis compañeros, por enseñarme lo esencial de la vida y del amor. 


\section{PUBLICACIONES PARCIALES DEL PRESENTE TRABAJO DE TESIS}

\section{INTRODUCCIÓN GENERAL}

\section{Publicaciones en revistas internacionales}

- Blanco PG, Arias DO, Gobello C. 2008. Doppler ultrasound in canine pregnancy. J Ultrasound Med 27 (12):1745-50.

\section{CAPÍTULO I}

\section{Publicaciones en revistas internacionales}

- Blanco PG, Tórtora M, Rodríguez R, Arias D, Gobello C. 2010. Ultrasonographic assessment of maternal cardiac function and peripheral circulation during normal gestation in dogs. Vet $\mathbf{J}$ (en prensa).

\section{Resúmenes presentados en congresos internacionales y nacionales}

- Blanco PG, Rube A, Re N, Gómez F, Tórtora M, Rodríguez R, Arias D, Gobello C. Doppler ultrasonographic assessment of maternal cardiac function and peripheral circulation during normal canine gestation. En Proceedings CD: Quinta Conferencia Latinoamericana de Veterinaria, organizada por el Latin American Veterinary Conference del 16 al 19 de octubre de 2009. Lima, Perú.

- Blanco PG, Rube A, Giacomone J, Gómez F, Prío V, Rodríguez R, Arias D, Gobello C. Ultrasonografía Doppler en la evaluación de la función cardíaca y la circulación 
periférica materna durante la gestación canina normal. Presentado en el IX Congreso Nacional de AVEACA. Organizado por esta Asociación en la Ciudad Autónoma de Bs. As., los días 1 y 2 de Octubre de 2009.

- Blanco, P; Rube, A; Prio, V; Tortora, M; Rodríguez, R; Corrada, Y; Arias, D; Gobello, C. Variaciones de Parámetros Ecocardiográficos en la Perra Gestante: Reporte Preliminar. Presentado en el VII Congreso Nacional de AVEACA. Organizado por esta Asociación en la Ciudad Autónoma de Bs. As., los días 23 y 24 de Septiembre de 2007.

\section{CAPÍTULO II}

\section{Publicaciones en revistas internacionales}

- Blanco PG, Arias D, Rube A, Barrena JP, Corrada Y, Gobello C. 2009. An experimental model to study resistance index and systolic/diastolic ratio of uterine arteries in adverse canine pregnancy outcome. Reprod Domest Anim 44: 164-166.

\section{Resúmenes presentados en congresos internacionales y nacionales}

- Blanco P, Arias D, Rube A, Barrena JP, Corrada Y, Gobello C. 2008. An experimental model to study resistance index and systolic/diastolic ratio of uterine arteries in adverse canine pregnancy outcome. En Proceedings Book: 6th International Symposium on Canine and Feline Reproduction. Vienna, Austria pp 45-46.

- Blanco P, Rube A, Barrena JP, Prío V, Cruz A, Gobello C, Tórtora M, Arias D. Un Modelo experimental para el estudio del índice de resistencia y la relación S/D de las 
arterias uterinas en la gestación canina anormal. Presentado en el VIII Congreso Nacional de AVEACA. Organizado por esta Asociación en la Ciudad Autónoma de Bs. As., los días 14 y 15 de Agosto de 2008.

\section{CAPÍTULO III}

\section{Publicaciones en revistas internacionales}

- P.G. Blanco, R. Rodríguez, A. Rube, D. Arias, M. Tórtora, J.D. Díaz, C. Gobello. 2011. Doppler ultrasonographic assessment of maternal and fetal blood flow in abnormal canine pregnancy. Anim Reprod Sci (en prensa).

\section{Resúmenes presentados en congresos internacionales y nacionales}

- Blanco PG, Rube A, Prío V, Pons E, Arias D, Gobello C. 2010. Doppler ultrasonographic assessment of uterine artery in abnormal canine pregnancy: preliminar report. En Proceedings Book: 7th European Veterinary Society for Small Animal Reproduction Congress. Louvain-La-Neuve, Bélgica. pp 104.

- Blanco PG, Rube A, Giacomone J, Cruz A, Prío V, Arias D, Gobello C. Índice de resistencia de las arterias uterinas en la gestación canina normal: reporte preliminar. Presentado en las $6^{\circ}$ Jornadas Internacionales de Veterinaria Práctica, pequeños, grandes animales y bromatología, organizadas por el Colegio de Veterinarios de la Provincia de Buenos Aires en la ciudad de Mar de Plata. 14 y 15 de Agosto de 2009.

- P Blanco; Y Corrada; R Rodríguez; D Arias y C Gobello. 2006. Ultrasonografía Doppler en la evaluación de parámetros cardiovasculares materno-fetales en perras 
gestantes normales: reporte preliminar. Memorias VI Congreso Nacional de AVEACA, 23 y 24 de septiembre de 2006. Buenos Aires, Argentina. Pp 121. Con referato.

\section{CAPÍTULO IV}

\section{Publicaciones en revistas internacionales}

- Blanco PG, Batista PR, Gómez FE, Pons ER, Arias DO, Gobello C. 2010.

Echocardiographic and Doppler assessment of maternal cardiovascular function during abnormal pregnancy in dogs. Vet $\mathbf{J}$ (enviado).

\section{Resúmenes presentados en congresos internacionales y nacionales}

- Blanco P, Rube A, Batista P, Rodríguez R, Barrena JP, Aprea A, Arias D, Gobello C. 2010. Evaluación ecocardiográfica y Doppler de la función cardiovascular materna en la gestación canina anormal. Conferencia Veterinaria Latinoamericana, organizada por el Latin American Veterinary Conference 27 de octubre de 2010. Lima, Perú.

\section{CAPÍtulO V}

\section{Publicaciones en revistas internacionales}

- PG Blanco, PR Batista, NE Re, DO Arias and C Gobello. 2011. Electrocardiographic changes in normal and abnormal canine pregnancy. Reprod Domest Anim (enviado). 


\section{Resúmenes presentados en congresos internacionales y nacionales}

- P.G. Blanco, P.R. Batista, M. Tórtora, R. Rodríguez, D.O. Arias, C. Gobello. Electrocardiographic monitoring in normal and abnormal pregnant dogs. 21st ECVIMCA Annual Congress 2011, Sevilla, España (enviado).

- Blanco P, Rube A, Prío V, Tórtora M, Rodríguez R, Corrada Y, Gobello C, Arias D. 2007. Parámetros electrocardiográficos pre, pos y gestacionales en la perra: Reporte Preliminar. $5^{\circ}$ Jornadas Internacionales de Veterinaria Práctica, pequeños, grandes animales y bromatología, organizadas por el Colegio de Veterinarios de la Provincia de Buenos Aires en la ciudad de Mar de Plata, 10 y 11 de agosto de 2007. Con referato. 


\section{ÍNDICE DE CONTENIDOS}

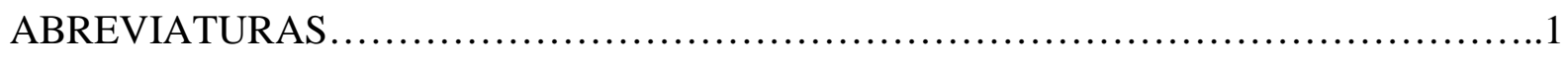

RESUMEN.................................................................

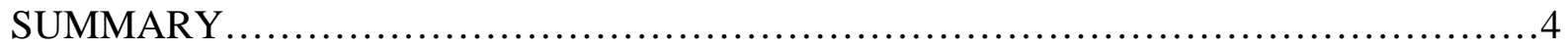

INTRODUCCIÓN GENERAL........................................................6

CAPÍTULO I: Evaluación ultrasonográfica de la función cardíaca y la circulación periférica

maternas durante la gestación canina normal........................................16

CAPÍTULO II: Un modelo experimental para el estudio cardiovascular materno - fetal del desenlace gestacional adverso en caninos....................................... 37

CAPÍTULO III: Evaluación ultrasonográfica Doppler del flujo sanguíneo materno - fetal en la

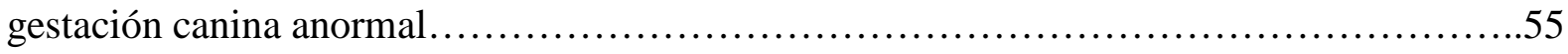

CAPÍTULO IV: Evaluación ultrasonográfica de la morfología y función cardíaca y la circulación periférica maternas durante la gestación canina anormal......................73 CAPÍTULO V: Cambios electrocardiográficos en la gestación canina normal y anormal.....89

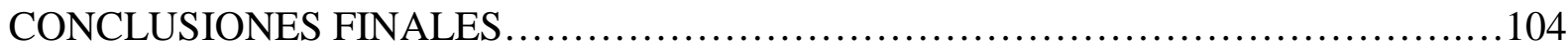


ABREVIATURAS

ACTH: Adecorticotrofina

ANOVA: Análisis de varianza

EE: Error estándar de la media

FC: Frecuencia cardíaca

kg: Kilogramo

LH: Hormona luteinizante

lpm: Latidos por minuto

MHz: Megahertz

$\mathrm{mm} / \mathrm{s}$ : Milímetro por segundo

mV: Milivoltios

VI: Ventrículo izquierdo

VTI: Integral de velocidad y tiempo 


\section{APLICABILIDAD DE LA ULTRASONOGRAFÍA DOPPLER Y ELECTROCARDIOGRAFÍA EN LA EVALUACIÓN DE PARÁMETROS CARDIOVASCULARES MATERNO-FETALES NORMALES Y PATOLÓGICOS EN LA GESTACIÓN CANINA}

Palabras clave: Ultrasonografía, Doppler, gestación, cardiovascular, perra, aborto.

Resumen:

El objetivo del presente Trabajo de Tesis fue evaluar, los cambios cardiovasculares maternos y fetales mediante ultrasonografía Doppler y electrocardiografía, durante la gestación canina normal, en un modelo experimental de anormalidad gestacional y en gestaciones anormales espontáneas. Se utilizó un total de 43 perras que se dividieron retrospectivamente en 4 grupos: preñadas normales $(n=12)$, vacías en diestro $(n=10)$, medicadas (grupo experimental; $n=10)$ y gestantes anormales, con interrupción espontánea de la gestación o mortalidad perinatal $>60 \%$ $(\mathrm{n}=11)$. En todos los grupos se realizó ecocardiograma, electrocardiograma, medición de presión arterial sistémica y Doppler de arterias uterinas. En las hembras preñadas también se realizó Doppler y ecocardiografía fetal. Los grupos se evaluaron cada 10 días, excepto el grupo medicado, que se evaluó cada 3 días. Los datos obtenidos se analizaron por ANOVA de medidas repetidas y una prueba de Chi cuadrado, según las variables analizadas. Durante la gestación canina normal, la fracción de acortamiento ( $\mathrm{p}<0,01)$, el gasto cardíaco $(\mathrm{p}<0,01)$ y la frecuencia cardíaca materna $(\mathrm{p}<0,01)$ se incrementaron, mientras que la presión arterial sistémica $(\mathrm{p}<0,05)$ y el índice de resistencia de la arteria uterina $(\mathrm{p}<0,01)$ descendieron. El grupo medicado presentó valores de presión arterial sistémica $(\mathrm{p}<0,05)$ y de índice de resistencia de la arteria uterina $(\mathrm{p}<0,01)$ mayores al de las gestantes normales. Las gestantes 
anormales espontáneas difirieron con las normales en los índices de resistencia de las arterias uterina $(\mathrm{p}<0,01)$, umbilical $(\mathrm{p}<0,05)$ y renal fetal $(\mathrm{p}<0,05)$, fracción de acortamiento $(\mathrm{p}<0,05)$, estrés de fin de sístole $(\mathrm{p}<0,01)$ y frecuencia cardíaca materna $(\mathrm{p}<0,01)$. Se concluye que en los caninos, los parámetros cardiovasculares maternos y fetales se modifican durante la preñez normal y patológica y constituyen predictores de anomalías obstétricas, contribuyendo al diagnóstico y prevención del resultado gestacional adverso. 


\section{APPLICABILITY OF DOPPLER ULTRASOUND AND ELECTROCARDIOGRAPHY IN THE EVALUATION OF NORMAL AND PATHOLOGICAL MATERNAL AND FETAL CARDIOVASCULAR PARAMETERS IN CANINE GESTATION}

Key words: Ultrasound, Doppler, gestation, cardiovascular, dog, abortion.

Summary:

The aim of this Thesis was to assess maternal and fetal cardiovascular changes, by Doppler ultrasound and electrocardiography, in normal and abnormal gestation as well as in an experimental model of abnormal pregnancy in the dog. Forty-three bitches were retrospectively assigned to one of the following groups: normal pregnant $(n=12)$, diestrous non-pregnant $(\mathrm{n}=10)$, experimental (medicated group; $\mathrm{n}=10)$ and abnormal pregnant (spontaneous interruption of pregnancy or perinatal death $>60 \% ; n=11$ ). Echocardiography, electrocardiogram, systolic blood pressure measurement and Doppler of uterine arteries were carried out in all the animals. In pregnant groups, fetal Doppler and echocardiography were also performed. The groups were evaluated every ten days, except the experimental group which was evaluated every three days from the beginning of the treatment. Comparisons among groups were performed by repeated measures ANOVA followed by Tukey comparison test and Chi square test depending on the analyzed variables. During normal gestation, shortening fraction $(\mathrm{p}<0.01)$, cardiac output $(\mathrm{p}<0.01)$ and maternal heart rate $(\mathrm{p}<0.01)$ increased, while systolic blood pressure $(\mathrm{p}<0.05)$ and resistance index of uterine artery $(\mathrm{p}<0.01)$ decreased. The experimental group showed higher systolic blood pressure $(\mathrm{p}<0.05)$ and resistance index of uterine artery $(\mathrm{p}<0.01)$ than the normal pregnant group. Uterine 
$(\mathrm{p}<0.01)$, umbilical $(\mathrm{p}<0.05)$ and fetal renal arteries $(\mathrm{p}<0.05)$ resistance indexes, shortening fraction $(\mathrm{p}<0.05)$, end systolic stress $(\mathrm{p}<0.01)$ and maternal heart rate $(\mathrm{p}<0.01)$ differed between the normal and the abnormal spontaneous pregnant groups. It is concluded that in dogs, maternal and fetal cardiovascular parameters change in the course of normal and pathological gestation. These parameters are good predictors of obstetric disease and contribute to the diagnosis and prevention of adverse pregnancy outcome. 


\section{INTRODUCCIÓN GENERAL}

El objetivo principal de cualquier programa de cría es lograr que una hembra saludable dé a luz cachorros sanos. Existen en la actualidad un número amplio de herramientas tecnológicas disponibles para aumentar la eficiencia reproductiva. No obstante, en áreas como la imagenología obstétrica o la electrocardiografía, aún no se han puesto a punto todas las tecnologías disponibles para el seguimiento y diagnóstico precoz de enfermedades.

Se sabe que en los mamíferos, los cambios cardiovasculares adaptativos durante la preñez tienen una importancia vital para la madre y el feto (1). Tanto la morfología como el funcionamiento cardíaco materno se alteran para garantizar un aporte sanguíneo eficaz al útero gestante. Se han detallado las modificaciones de la función diastólica (2), del gasto cardíaco (3), de la sensibilidad de los barorreceptores (4) y del pulso arterial (5), que tienen lugar en la mujer durante el embarazo sin complicaciones. Una de las principales variables que se controlan rutinariamente en el humano es la presión arterial sistémica, que normalmente desciende en este período (6). El método complementario de elección para la evaluación no invasiva del sistema cardiovascular es la ultrasonografía bidimensional, en modo M y Doppler (7). La ecocardiografía se ha aplicado ampliamente en el monitoreo de los parámetros anteriormente mencionados en medicina humana (8).

La aplicación de la ultrasonografía es un área de investigación que está alcanzando una importancia considerable en el campo de las ciencias veterinarias. Las ondas de ultrasonido tuvieron en primera instancia un uso industrial y más tarde comenzaron a utilizarse para examinar el interior del cuerpo humano. Desde que Yoshida y col. (1961) emplearon la 
ultrasonografía Doppler en el estudio de los movimientos cardíacos en 1961, la técnica ha sido sumamente utilizada en medicina humana (9). En la actualidad, los equipamientos son relativamente simples y permiten su uso rutinario en la clínica. Una de las mayores aplicaciones de la ultrasonografía es el campo de la obstetricia, debido a la facilidad con que se emiten y reciben ondas de ultrasonido a través del útero gestante $(10,11)$. Sumado a esta ventaja, el uso del ultrasonido de baja frecuencia no resulta perjudicial para la madre ni para el feto. Si bien se han llevado a cabo investigaciones sobre la dinámica circulatoria de la madre y el feto en diferentes especies (12-16), la ultrasonografía Doppler se ha desarrollado en humanos más que en cualquier otra especie. Desde 1968 (9), cuando la ultrasonografía Doppler fue usada por primera vez en obstetricia humana, ha contribuido al reconocimiento de ciertas circunstancias patológicas y al tratamiento de pacientes con diferentes enfermedades $(17,18)$. Utilizando esta técnica es posible evaluar con precisión el flujo sanguíneo de los vasos maternos (19) y fetales (20), así como también la circulación a nivel de las cámaras cardíacas maternas y fetales (21). Los vasos examinados rutinariamente en las mujeres embarazadas son las arterias uterinas, umbilical y cerebral media fetal.

Los patrones de velocidad del flujo sanguíneo de la arteria uterina proveen información sobre la adecuada erosión de las arterias espiraladas maternas por parte del trofoblasto (22). Estas arterias se convierten en vasos de baja resistencia como consecuencia de esa erosión. Los índices de resistencia decrecen a medida que la circulación distal se incrementa, aumentando la superficie del lecho vascular irrigado. Durante el segundo trimestre del embarazo, el índice de resistencia (IR) normal de la arteria uterina promedia valores de 0,34 , con un rango de referencia 0,15 a 0,5 (23-26). En la mujer, valores en el IR por encima de 0,58 a la semana 24 de gestación se asocian con un desenlace gestacional adverso (27-28). Por este motivo, a 
partir de la segunda mitad del embarazo, el examen ultrasonográfico Doppler juega un rol fundamental en la toma de decisiones terapéuticas.

La arteria umbilical también aporta información obstétrica vital. El flujo diastólico ausente o reverso o el incremento en el IR de esta arteria reflejan condiciones que afectan el aporte sanguíneo al feto. Un estudio demostró que los fetos que presentan ausencia de flujo diastólico en esta arteria, fallecen dentro de las 3 semanas de efectuado el diagnóstico (17). En la clínica de pequeños animales, la sonografía bidimensional ha sido utilizada para evaluar exitosamente la gestación canina (29-32). Se ha sugerido que la medición de algunos parámetros embrionales puede predecir una reabsorción inminente en las perras (30-33). La ultrasonografía Doppler empezó a usarse para monitorear la preñez canina en 1970, donde se identificó por primera vez el latido cardíaco fetal y la circulación sanguínea placentaria (34). El IR y el índice de pulsatilidad (IP) del flujo sanguíneo intraovárico se midieron durante el ciclo estral normal, la preñez y la luteólisis inducida por la prostaglandina $\mathrm{F}_{2} \alpha$ en perras Beagle. En el ovario, el flujo se acrecienta durante el proestro, es máximo en la ovulación y fase lútea temprana y declina con la regresión lútea (35).

Se han llevado a cabo mediciones de velocidad, IR y morfología de ondas pulsátiles Doppler de la arteria uterina en perras en anestro (36). Asimismo, estos parámetros vasculares se han evaluado en las arterias uteroplacentarias, umbilical y en la carótida común fetal, en un estudio preliminar en 6 perras Beagle preñadas (37). Conjuntamente, se midió el IP y la relación A:B de esos mismos vasos, mostrando las variaciones semanales que sufren estos valores durante la gesta (37). Con respecto a la forma de las ondas pulsátiles Doppler, se ha comprobado que las arterias uteroplacentarias, umbilicales y la aorta fetal sufren modificaciones a lo largo de la gestación, a diferencia de las venas umbilicales que se presentan prácticamente invariables (38). 
El sistema de conducción eléctrica del corazón acompaña todos los cambios mecánicos y hemodinámicos, contribuyendo con la adaptación cardíaca y circulatoria. La electrocardiografía es otro método complementario no invasivo que permite monitorear las diferencias en el trazado de la actividad cardíaca eléctrica durante la gestación (39). Esta técnica ha sido aplicada para examinar el aumento en la frecuencia cardíaca que tiene lugar durante esta etapa fisiológica, en la hembra canina (40). Sin embargo existen otros parámetros electrofisiológicos aún no descriptos que también reflejan el funcionamiento eléctrico del corazón.

Los escasos estudios previamente mencionados arrojan datos preliminares que sirven como punto de partida para posteriores investigaciones. La totalidad de los cambios cardiovasculares adaptativos fisiológicos que tienen lugar durante una preñez normal, no han sido descriptos en detalle en los caninos domésticos. Menos aún se conocen, en esta especie, las alteraciones patológicas más frecuentes de estos parámetros.

El desarrollo de métodos diagnósticos ha aumentado en obstetricia humana, resultando en el diagnóstico temprano de gestaciones patológicas o distrés fetal, lo que permite una intervención temprana (41). En este sentido, la ultrasonografía Doppler ha sido vital para distinguir los fetos hipóxicos en peligro de descompensación hemodinámica de aquellos estables (24). A pesar de que la ultrasonografía Doppler ha sido poco desarrollada en reproducción canina, los escasos reportes disponibles demuestran que existe en esta especie una tendencia similar a la encontrada en otras especies, incluso en humanos.

En la actualidad, la frecuencia cardíaca fetal, los movimientos y tamaño fetales son los únicos parámetros imagenológicos utilizados para diagnosticar una preñez patológica en perras. La 
toma de decisiones terapéuticas en obstetricia canina de importancia superlativa para la vida de la madre y de los fetos, como son la inducción del parto o la realización de la cesárea, se sustenta sólo con la medición de estas variables. Pero la alteración de las mismas es, en la mayoría de los casos, reflejo de un trastorno ya avanzado. Como consecuencia, el diagnóstico precoz de la gestación anormal es un logro infrecuente.

En consecuencia, resulta de gran interés para la clínica reproductiva canina, describir los cambios cardiocirculatorios maternos y fetales durante la preñez canina. Estos parámetros podrían constituir predictores de anomalías obstétricas en caninos, contribuyendo al diagnóstico e incluso prevención del resultado gestacional adverso antes de que otros signos clínicos o ecográficos aparezcan.

Por todo lo expuesto, los objetivos particulares del siguiente Trabajo de tesis:

- Describir los cambios en la morfología cardíaca, función sistólica y dos parámetros hemodinámicos periféricos maternos durante la gestación canina normal.

- Estudiar los cambios en la morfología cardíaca, función sistólica y circulación materna y fetal en un modelo experimental de gestación anormal farmacológicamente inducida.

- Reportar el flujo sanguíneo de la arteria uterina, arteria umbilical, aorta abdominal fetal, renal fetal y carótida interna fetal en la gestación canina anormal.

- Describir los cambios en la morfología cardíaca, función sistólica y presión arterial sistémica materna durante la gestación anormal en perros. 
- Analizar los cambios electrocardiográficos en el curso de la gestación normal y anormal en las perras.

\section{Bibliografía}

1. Eghbali M, Deva R, Alioua A, Minosyan TY, Ruan H, Wang Y, Toro L, Stefani E. Molecular and Functional Signature of Heart Hypertrophy During Pregnancy. Circ Res 2005;96:1208-16.

2. Escudero E, Favaloro L, Moreira C, Pisano O, Plastino J M, Coccozella G. Características de la función diastólica del ventrículo izquierdo en el embarazo: estudio ecocardiográfico. Revista Federación Argentina de Cardiología 1986;15:61-7.

3. Del Bene R, Barletta G, Mello G, Lazzeri C, Mecacci F, Parretti E, Martín E, Vecchiarino S, Franchi F, La Villa G. Cardiovascular function in pregnancy: effects of posture. Br J Obstet Gynecol 2001;108:344-52.

4. Voss A, Baumert M, Baier V, Stepan H, Walther T, Faber R. Autonomic cardiovascular control in pregnancies with abnormal uterine perfusion. Am J Hypertens 2006;19:306-12. 5. Smith SA, Morris JM, Gallery ED. Methods of assessment of the arterial pulse wave in normal human pregnancy. Am J Obstet Gynecol 2004;190:472-6.

6. Kametas NA, McAuliffe F, Hancock J, Chambers J, Nicolaides KH. Maternal left ventricular mass and diastolic function during pregnancy. Ultrasound Obstet Gynecol 2001;18:460-6. 
7. Blanco PG, Arias DO, Gobello C. Doppler ultrasound in canine pregnancy. J Ultrasound Med 2008;27:1745-50.

8. Valensise H, Novelli GP, Vasapollo B, Borzi M, Arduini D, Galante A, Romanini C. Maternal cardiac systolic and diastolic function: relationship with uteroplacental resistances. A Doppler and echocardiographic longitudinal study. Ultrasound Obstet Gynecol 2000;15:487-97.

9. Yoshida T, Mori M, Nimura Y, et al. Analysis of heartmotion with ultrasonic Doppler method and its clinicalapplication. Am Heart J 1961;61:61-75.

10. Bishop EH. Ultrasonic fetal monitoring. Clin Obstet Gynecol 1968;11:1154-64.

11. Fielder FD, Baker RF. Diagnostic applications of Doppler ultrasound. Ultrasonics $1969 ; 7: 36-8$

12. Bollwein H, Meyer HH, Maierl J, Weber F, Baumgartner U, Stolla R. Transrectal Doppler sonography of uterine bloodflow. Theriogenology 2000;53:1541-52.

13. Bollwein H, Baumgartner U, Stolla R. Transrectal Dopplersonography of uterine blood flow in cows during pregnancy.Theriogenology 2002;57:2053-61.

14. Bollwein H, Mayer R, Stolla R. Transrectal Doppler sonographyof uterine blood flow during early pregnancy inmares. Theriogenology 2003;60:597-605.

15. Bollwein H, Weber F, Woschée I, Stolla R. TransrectalDoppler sonography of uterine and umbilical blood flowduring pregnancy in mares. Theriogenology 2004;61:499-509.

16. Reynolds LP, Caton JS, Redmer DA. Evidence for altered placental blood flow and vascularity in compromised pregnancies. J Physiol 2006;572:51-8.

17. Detti L, Akiyama M, Mari G. Doppler blood flow in obstetrics. Curr Opin Obstet Gynecol 2002;14:587-93. 
18. Robson SC. Assessment of hemodynamics using Doppler ultrasound. Ultrasound Obstet Gynecol 2000;15:456-9.

19. Fitzgerald DE, Drumm JE. Non-invasive measurement ofhuman fetal circulation using ultrasound: a new method. Br Med J 1977;2:1450-1.

20. Maulik D, Nanda NC, Saini VD. Fetal Doppler echocardiography: methods and characterization of normal and abnormalhemodynamics. Am J Cardiol 1984;53:572-8.

21. Easterling TR, Watts DH, Schmucker BC, Benedetti TJ. Measurement of cardiac output during pregnancy: validation of Doppler technique and clinical observations in preeclampsia. Obstet Gynecol 1987;69:845-50.

22. Campbell S, Pearce JM, Hackett G, Cohen-Overbeek T, Hernandez C. Qualitative assessment of uteroplacentalblood flow: early screening test for high-risk pregnancies. Obstet Gynecol 1986;68:649-53.

23. Dickey RP. Doppler ultrasound investigation of uterine and ovarian blood flow in infertility and early pregnancy. Hum Reprod Update 1997;3:467-503.

24. Filkins K, Koos JB. Ultrasound and fetal diagnosis. Curr Opin Obstet Gynecol 2005;17:185-95.

25. Papageorghiou AT, Yu CK, Nicolaides KH. The role of uterineartery Doppler in predicting adverse pregnancy outcome. Best Pract Res Clin Obstet Gynaecol 2004;18:383-96. 26. Deurloo KL, Spreeuwenberg MD, Bolte AC, Van Vugt JM. Color Doppler ultrasound of spiral artery blood flow forprediction of hypertensive disorders and intrauterine growth restriction: a longitudinal study. Prenat Diagn 2007;27:1011-6.

27. Strigini FA, Lencioni G, De Luca G, Lombardo M, Bianchi F, Genazzani AR. Uterine artery velocimetry and spontaneous preterm delivery. Obstet Gynecol 1995;85:374-7. 
28. Coleman MA, McCowan LM, North RA. Mid-trimester uterine artery Doppler screening as a predictor of adversepregnancy outcome in high-risk women. Ultrasound Obstet Gynecol 2000;15:7-12.

29. Yeager AE, Concannon PW. Association between the preovulatory luteinizing hormone surge and the early ultrasonographicdetection of pregnancy and fetal heart beats in beagle dogs. Theriogenology 1990;34:655-65.

30. England GC. Ultrasonographic assessment of abnormal pregnancy. Vet Clin North Am Small Anim Pract 1998;28:849-68.

31. Kim Y, Travis AJ, Meyers-Wallen VN. Parturition predictionand timing of canine pregnancy. Theriogenology 2007;68:1177-82.

32. Kutzler MA, Mohammed HO, Lamb SV, Meyers-WallenVN. Accuracy of canine parturition date prediction from the initial rise in preovulatory progesterone concentration. Theriogenology 2003;60:1187-96.

33. England GC, Russo M. Ultrasonographic characteristics of early pregnancy failure in bitches. Theriogenology 2006;66:1694-8.

34. Helper LC. Diagnosis of pregnancy in the bitch with an ultrasonic Doppler instrument. J Am Vet Med Assoc 1970;156:60-2.

35. Köster K, Poulsen Nautrup C, Günzel-Apel AR. A Doppler ultrasonographic study of cyclic changes of ovarian perfusion in the Beagle bitch. Reproduction 2001;122:453-61. 36. Alvarez-Clau A, Liste F. Ultrasonographic characterizationof the uterine artery in the nonestrus bitch. Ultrasound Med Biol 2005;3:1583-7.

37. Nautrup CP. Doppler ultrasonography of canine maternaland fetal arteries during normal gestation. J Reprod Fertil 1998;112:301-14. 
38. Di Salvo P, Bocci F, Polisca A. Doppler evaluation of maternal and fetal vessels during normal gestation in the bitch. Res Vet Sci 2006;81:382-8.

39. Baumert M, Seeck A, Faber R, Nalivaiko E, Voss A. Longitudinal changes in QT interval variability and rate adaptation in pregnancies with normal and abnormal uterine perfusion. Hypertens Res 2010;33:555-60.

40. Olsson K, Lagerstedt AS, Bergström A, Häggström J. Change of diurnal heart rate patterns during pregnancy and lactation in dogs (Canis familiaris). Acta Vet Scand 2003;44:105-10.

41. Buczinski SM, Fecteau G, Lefebvre RC, Smith LC. Fetal wellbeing assessment in bovine near-term gestations: current knowledge and future perspectives arising from comparative medicine. Can Vet J 2007;48:178-83. 


\section{CAPÍTULO I}

\section{EVALUACIÓN ULTRASONOGRÁFICA DE LA FUNCIÓN CARDÍACA Y LA CIRCULACIÓN PERIFÉRICA MATERNAS DURANTE}

\section{LA GESTACIÓN CANINA NORMAL}

\section{Introducción}

Los cambios cardiovasculares que se suceden durante la preñez normal se han descripto en humanos y ratones (1-3). La adaptación cardiovascular materna durante este período garantiza un óptimo desarrollo de los fetos. La ecocardiografía bidimensional, en modo M y Doppler ha sido ampliamente utilizada en medicina humana para evaluar la función sistólica y los cambios morfológicos que va sufriendo el VI a lo largo de la gestación fisiológica $(1,4)$. En las mujeres, la sobrecarga de volumen en el curso del embarazo induce una hipertrofia cardíaca con el correspondiente aumento de la masa ventricular (MV) y un incremento del gasto cardíaco $(4,5)$. En esta especie se ha reportado contradictoriamente que los índices de función del VI, incluyendo el porcentaje medio de acortamiento circunferencial (\%MAC) y fracción de acortamiento (FA), pueden incrementarse, mantenerse constantes o incluso disminuir durante el embarazo (6-8). Sin embargo, en ratones se ha indicado que la hipertrofia cardíaca durante este período se acompaña por un descenso de la FA, indicativo de una caída de la función sistólica (2). Por todo esto, no queda claro si la hipertrofia cardíaca gestacional se acompaña o no de una mejora en la función sistólica en todas las especies.

Asimismo, en la mujer embarazada la presión arterial sistémica (PAS) disminuye durante la gestación hasta alcanzar un mínimo hacia la mitad de este período (4). Esta disminución de la 
resistencia periférica está asociada a una caída en el índice de resistencia (IR) de la arteria uterina, garantizando un adecuado aporte de flujo sanguíneo al útero para el desarrollo fetal (4).

Una mala adaptación cardiovascular materna está estrechamente vinculada con un desenlace gestacional adverso (9-11). Existe evidencia de que las gestaciones complicadas con restricción de crecimiento intrauterino se asocian con una inadecuada expansión del espacio intravascular materno y una falta de incremento del gasto cardíaco (GC; 4). Más aún, el incremento de la presión arterial materna produce preeclampsia, un trastorno hipertensivo de la gestación en el cual el IR de la arteria uterina aumenta al igual que la presión arterial sistémica, provocando una perfusión fetal deficiente (12).

El estudio de la adaptación cardiovascular materna durante la gestación asegura una aproximación a la interacción entre la homeostasis materna y fetal y provee una herramienta útil en la detección de complicaciones. La información disponible sobre la función cardíaca durante la gestación canina normal es limitada. En esta especie, el diámetro diastólico del VI (DDVI), el volumen sistólico (VS) y el GC se encuentran elevados al día 50 y 60 de preñez (13). Sin embargo, no existe información sobre el comportamiento de estos parámetros a lo largo de todo el período gestacional, especialmente en la preñez temprana y media. El aumento reflejo de la FC junto con la actividad de la renina plasmática, las concentraciones de vasopresina, angiotensina II, ACTH y cortisol, se encuentran atenuadas durante la gestación canina (14).

La presión sanguínea se medió al día 21-28, 40 y 60 de preñez en perros (15). La circulación periférica ha sido evaluada a través del flujo sanguíneo uterino a partir de la tercer y cuarta semana de la gestación normal $(16,17)$. Sin embargo, se desconocen las características de la circulación uterina durante las primeras 3 semanas de preñez. Por todo lo expuesto, el 
objetivo de este estudio fue describir los cambios en la morfología cardíaca, función sistólica y dos parámetros hemodinámicos periféricos durante la gestación canina normal.

\section{Materiales y métodos}

\section{Animales y seguimiento}

Veinte perras saludables, de 1 a 4 años de edad, de 3 a $38 \mathrm{~kg}$ de peso, mestizas y de razas puras, preñadas $(\mathrm{PP} ; \mathrm{n}=10)$ y no preñadas $(\mathrm{NP} ; \mathrm{n}=10)$ se incluyeron en este estudio. La preñez fue confirmada ecográficamente en todos los casos (18). Las perras fueron evaluadas por ecocardiografía y ultrasonografía Doppler cada 10 días, desde el día 0 del ciclo estral hasta el parto o hasta el día 65 del ciclo para los grupos PP y NP, respectivamente. El día 0 del ciclo estral fue definido como el primer día de citología vaginal típica de estro (19). Este estudio fue aprobado por el Comité de Cuidado y Uso Animal de esta Institución.

\section{Citología vaginal}

Los animales se evaluaron diariamente para detectar la presencia de tumefacción vulvar y descarga vulvar sanguinolenta, fenómeno que se consideró indicativo del inicio del proestro. A partir de ese momento, se obtuvieron citologías vaginales diariamente hasta que indicaron el inicio del estro, que se estimó como el día de pico de LH. Los extendidos se tiñeron usando Tinción 15 stain (Biopur, Rosario; Argentina), se calcularon los porcentaje de los diferentes tipos de células y se interpretaron según reportes previos (19). 


\section{Evaluaciones ecocardiográficas}

Las evaluaciones ecocardiográficas bidimensionales, en modo M y Doppler se realizaron con los animales en estación, utilizando un transductor $7 \mathrm{MHz}$ (Toshiba Core Vision Pro; 20). Sobre la piel rasurada, se aplicó gel para lograr un adecuado acomplamiento acústico. Las ventanas paraesternales utilizadas fueron las previamente descriptas por Thomas y col. (1996; 21).

Se registraron las medidas de DDVI, diámetro sistólico del VI (DSVI), tabique interventricular en diástole (TIVD) y sístole (TIVS), pared posterior del VI en diástole (PPD) y sístole (PPS) y FA, por medio del modo M (22). La FA se calculó como (DDVIDSVI)/DDVI x 100 (23). La MV, relación radio espesor (RRE), estrés de fin de sístole (EFS) y \% MAC se calcularon de acuerdo a las fórmulas correspondientes (23-25). Con el mismo modo ecocardiográfico, se registraron la relación Ao/AI (Ao/AI), período eyectivo (PE), amplitud aórtica (AA) y punto de separación septal E (PSSE; 24).

Con ecocardiografía Doppler espectral se registró el VTI del flujo transaórtico a partir del cual se calcularon el VS y el GC $(23,26)$.

Para minimizar la variabilidad en las mediciones, todas fueron efectuadas por un único operador entrenado y siguiendo guías de recomendación específicas (21,27). Se promediaron tres valores de cada parámetro. 


\section{Presión arterial sistémica}

La PAS se midió utilizando un equipo Doppler vascular (DV610 Medmega, Brasil). Se utilizaron manguitos cuyo ancho correspondió al $40 \%$ de la circunferencia total del miembro anterior (28). Los animales se colocaron en decúbito lateral y se promediaron tres registros.

Evaluación del flujo sanguíneo uterino

El IR de la arteria uterina se evaluó con Doppler pulsado (Toshiba Core Vision Pro) con un transductor cónvex de 3,7-6 MHz. Las perras se colocaron en decúbito lateral. De esa forma, se obtuvieron los espectros según estudios previos (29). El IR se definió como (VMAX VMIN)/VMAX, donde VMAX corresponde a la velocidad de pico sistólico y VMIN a la velocidad de flujo de fin de diástole (30). Se registraron tres ondas uniformes y consecutivas para calcular el IR.

\section{Análisis estadístico}

Con el fin de constatar la comparabilidad de ambos grupos en función de la edad y peso, se efectuó un test de Student. Todas las variables fueron transformadas a porcentaje de cambio ([Valor final-Valor inicial / Valor inicial] x 100) y comparadas entre los grupos por medio de un ANOVA de medidas repetidas seguido de un test de Tukey (SPSS 17.0, SPSS Inc.). 


\section{Resultados}

Ambos grupos de animales fueron similares considerando edad y peso ( $p>0,1)$. Al comienzo del estudio, los parámetros cardiovasculares no difirieron entre los grupos $(\mathrm{p}>0,1)$. Todas las perras presentaron valores dentro de los rangos normales para su peso (31-33). Las Figuras 1 y 2 muestran registros ecocardiográfico en modo M y ultrasonográfico con Doppler pulsado de la arteria uterina, respectivamente. Se encontró una interacción entre el tiempo y el grupo para el porcentaje de cambio de PPS ( $p<0,05$; Figura 3), \%MAC ( $<<0,01$; Figura 4), FA $(\mathrm{p}<0,01$; Figura 5), VS ( $p<0,05$; Figura 6), GC $(\mathrm{p}<0,01$; Figura 7$)$ y FC $(\mathrm{p}<0,01$; Figura 8) a lo largo del estudio. El \%MAC y la FA empezaron a incrementarse desde el día 30 de preñez en adelante. La PPS aumentó desde el día 50 al 60, mientras que el VS, GC y la FC se incrementaron a partir del día 40 en adelante.

Asimismo, durante este estudio, se encontró una interacción entre tiempo y grupo para el porcentaje de cambio del EFS ( $p<0,05$; Figura 9), PAS ( $<<0,05$; Figura 10) e IR ( $p<0,01$; Figura 11). En el grupo PP estas variables disminuyeron progresivamente desde el día 30 de gestación hasta el parto.

Los porcentajes de cambio de DDVI, DSVI, TIVD y TIVS difirieron significativamente entre ambos grupos de perras (Tabla 1), en tanto que los porcentajes de cambio de VM, RRE, Ao/AI, PE, AA y PSSE no se modificaron a lo largo del experimento ( $\mathrm{p}>0,1$; Tabla 2). 


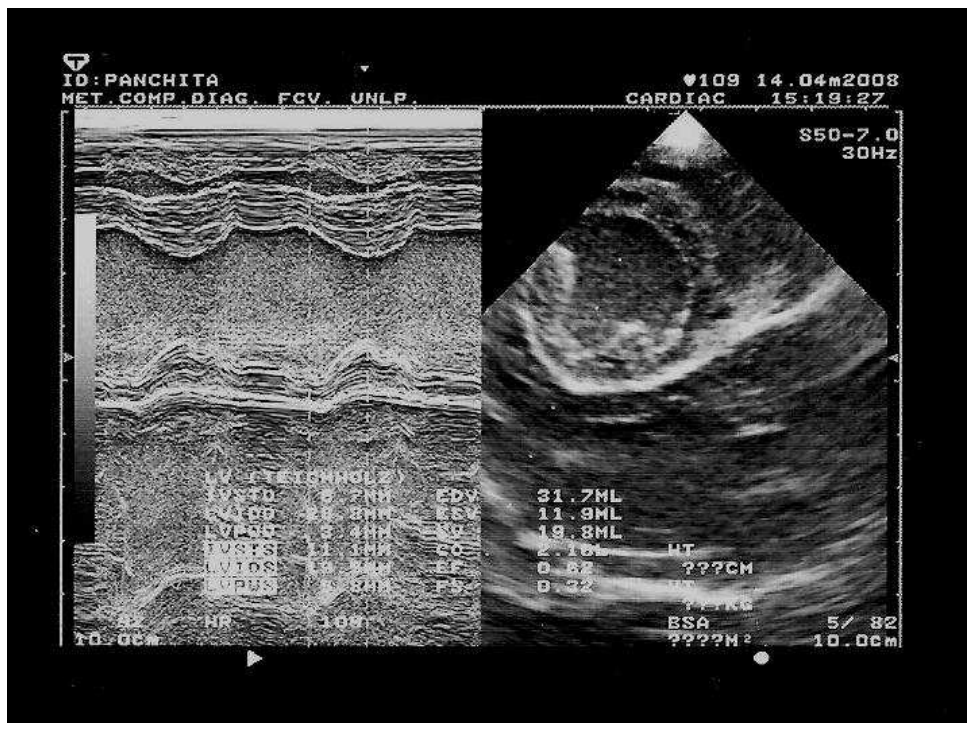

Figura 1: Imagen ecocardiográfica en modo M de una vista del VI en eje corto obtenida desde la ventana paraesternal derecha en una perra preñada, a los 60 días del pico estimado de LH.

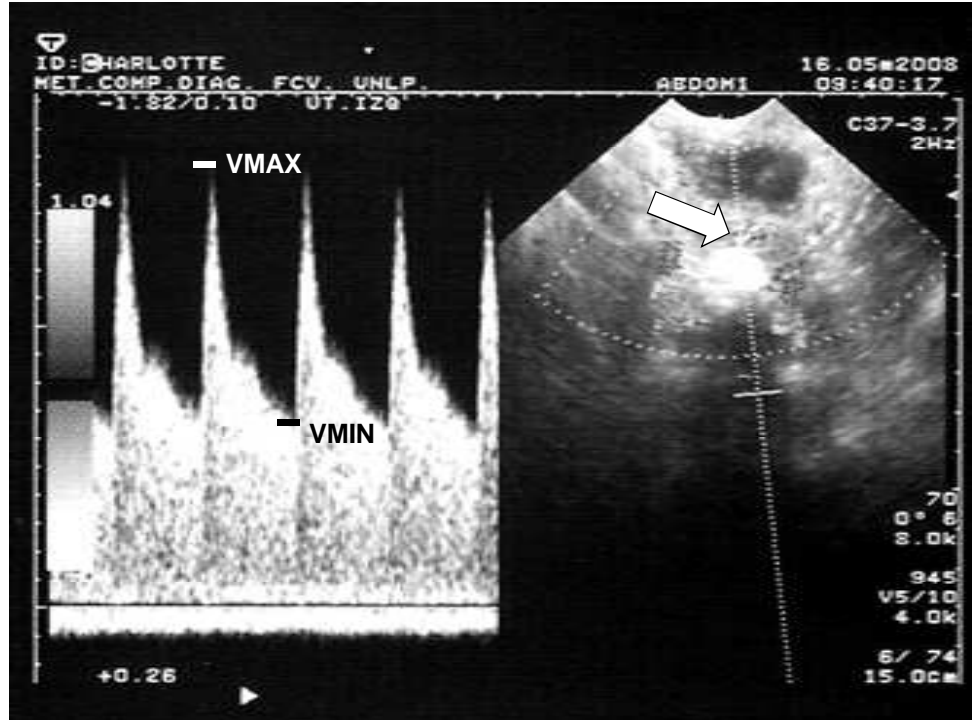

Figura 2: Registro ultrasonográfico con Doppler pulsado de la arteria uterina (flecha blanca) en una perra preñada, a los 60 días del pico estimado de LH. VMAX corresponde a la velocidad de pico sistólico y VMIN a la velocidad de flujo de fin de diástole. 


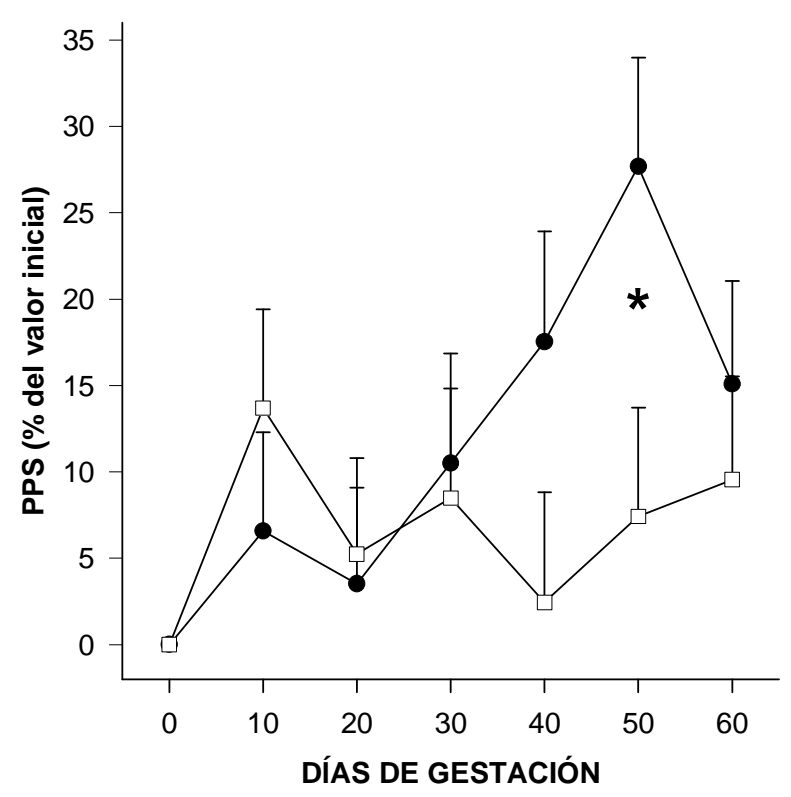

Figura 3: Porcentaje de cambio de la pared posterior en sístole (PPS; media \pm EE) de 10 perras preñadas (círculos) y 10 perras no preñadas (cuadrados) evaluadas cada 10 días durante 2 meses. El día 0 fue el día estimado del pico de LH.

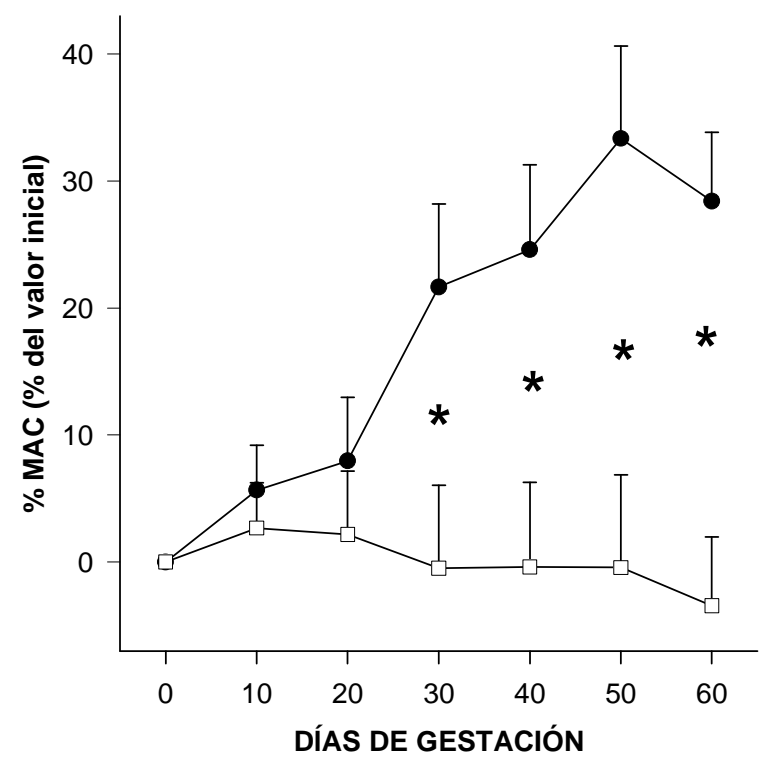

Figura 4: Porcentaje de cambio del porcentaje medio de acortamiento fraccionario (\%MAC; media $\pm E E$ ) de los mismos animales y experimento de la Figura 3. 


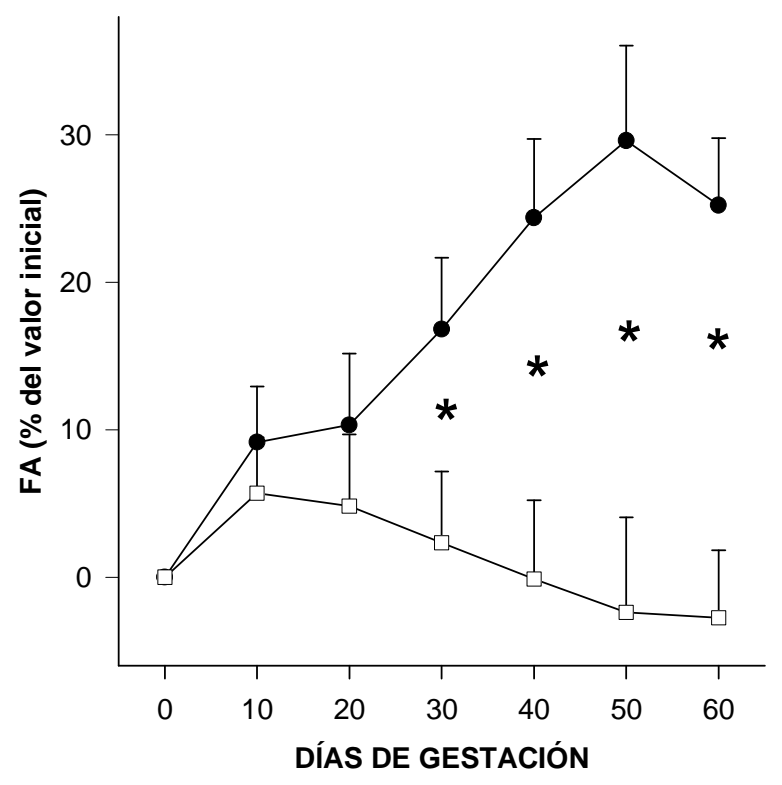

Figura 5: Porcentaje de cambio de la fracción de acortamiento (FA; media $\pm E E)$ de los mismos animales y experimento de la Figura 3.

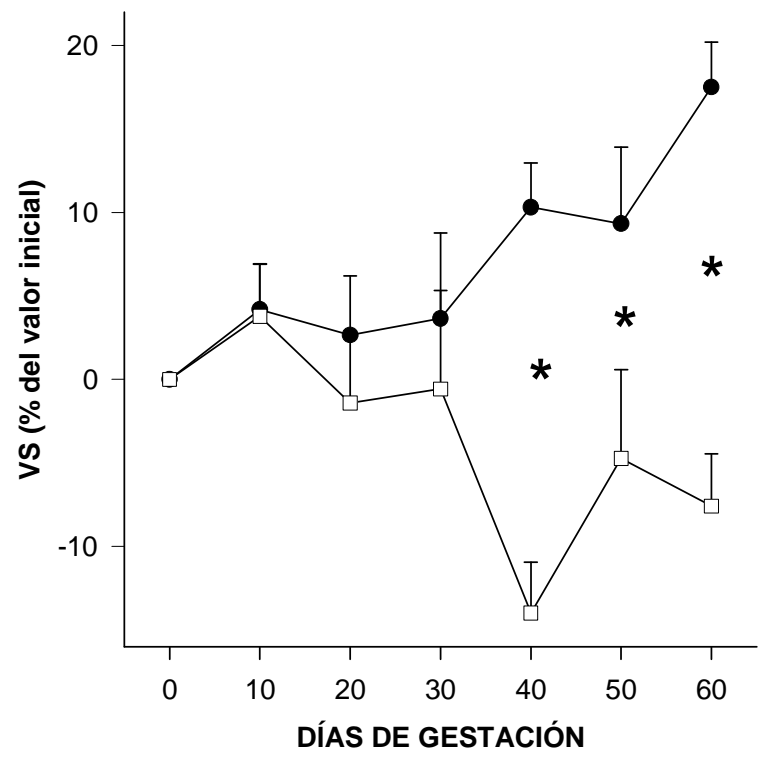

Figura 6: Porcentaje de cambio del volumen sistólico (VS; media \pm EE) de los mismos animales y experimento de la Figura 3. 


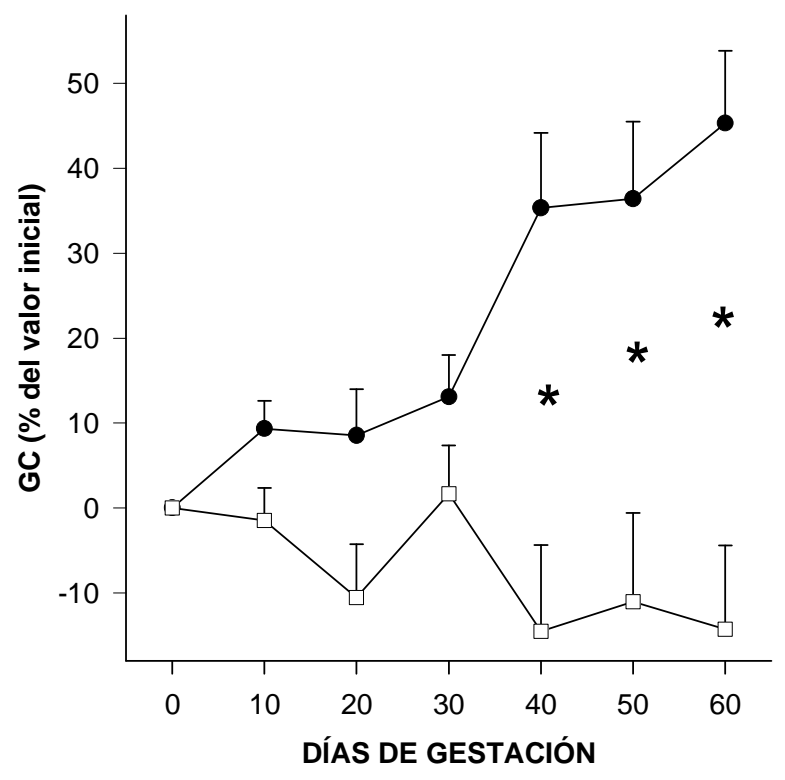

Figura 7: Porcentaje de cambio del gasto cardíaco (GC; media \pm EE) de los mismos animales y experimento de la Figura 3.

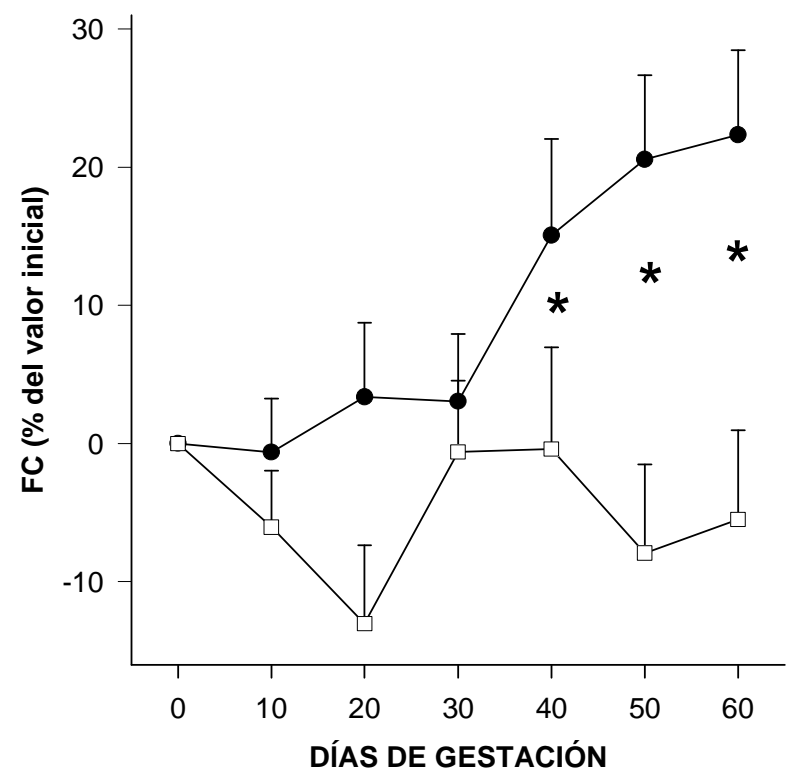

Figura 8: Porcentaje de cambio de la frecuencia cardíaca (FC; media $\pm E E)$ de los mismos animales y experimento de la Figura 3. 


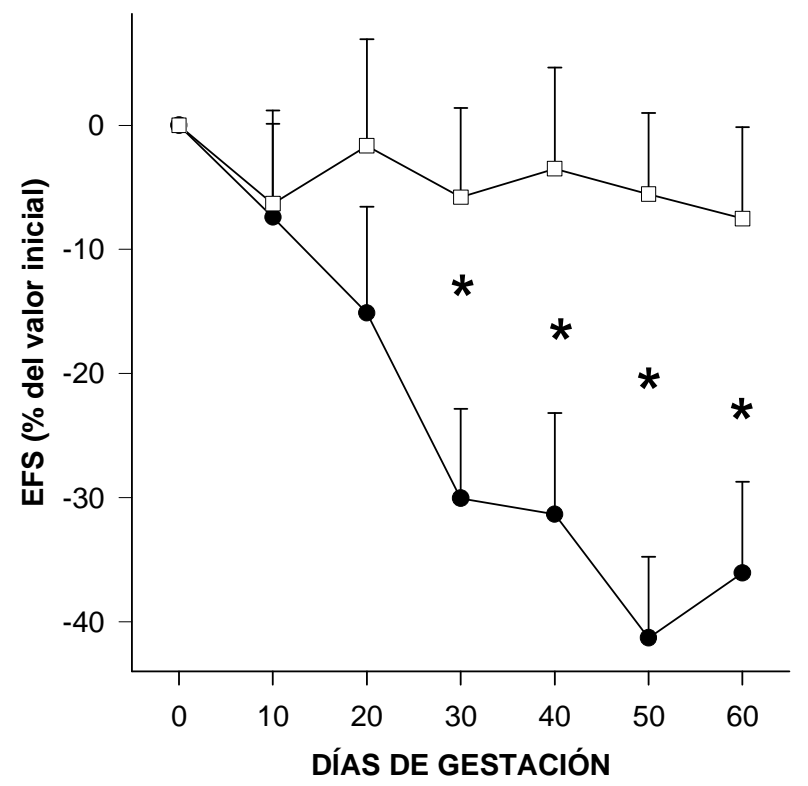

Figura 9: Porcentaje de cambio del estrés de fin de sístole (EFS; media $\pm E E)$ de los mismos animales y experimento de la Figura 3.

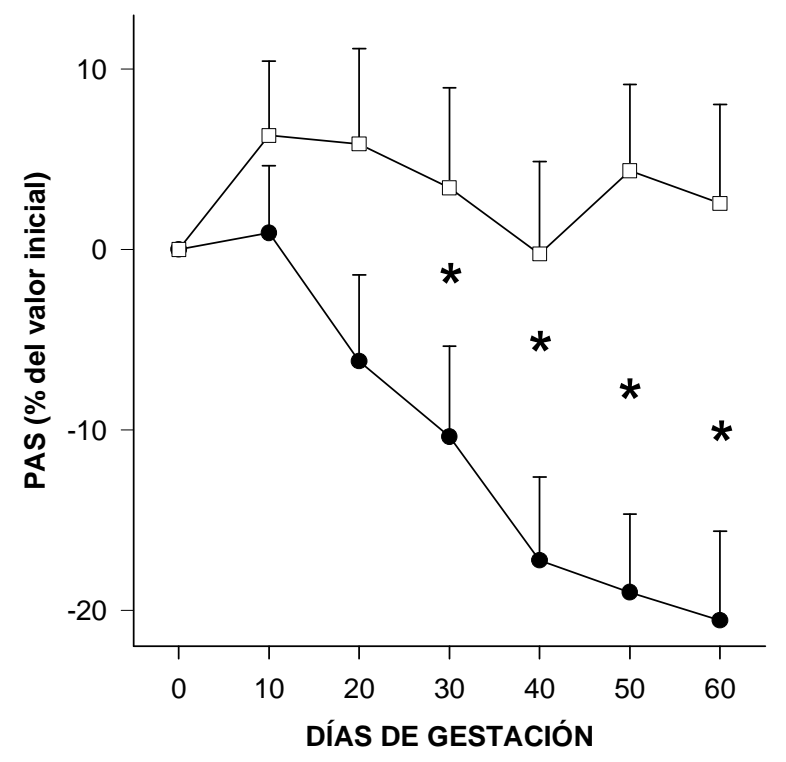

Figura 10: Porcentaje de cambio de la presión arterial sistémica (PAS; media EEE) de los mismos animales y experimento de la Figura 3. 


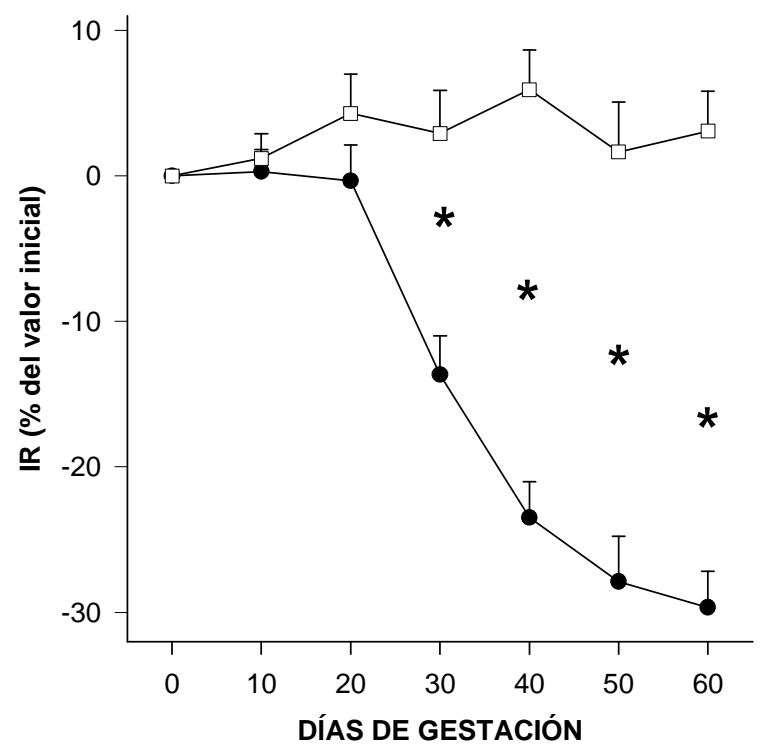

Figura 11: Porcentaje de cambio del índice de resistencia (IR; media $\pm E E)$ de los mismos animales y experimento de la Figura 3. 
Tabla 1: Porcentaje de cambio de parámetros ecocardiográficos (media $\pm E E$ ) de 10 perras preñadas (PP) y 10 perras no preñadas (NP) evaluadas cada 10 días durante 2 meses desde el día estimado del pico de LH.

\begin{tabular}{|c|c|c|c|c|c|c|}
\hline \multicolumn{7}{|c|}{ Día de gestación } \\
\hline & 10 & 20 & 30 & 40 & 50 & 60 \\
\hline \multicolumn{7}{|c|}{ TIVD $^{\mathrm{a}}$} \\
\hline PP & $8,5 \pm 3,9$ & $11,7 \pm 3,7$ & $7,8 \pm 5,2$ & $6,8 \pm 5,3$ & $10,3 \pm 5,4$ & $9,1 \pm 5,7$ \\
\hline NP & $1 \pm 3,9$ & $-1,7 \pm 3,7$ & $-0,6 \pm 5,2$ & $-12,1 \pm 5,3$ & $0,7 \pm 5,4$ & $-2,4 \pm 5$ \\
\hline \multicolumn{7}{|c|}{ TIVS $^{\mathrm{a}}$} \\
\hline $\mathrm{PP}$ & $6,8 \pm 3,7$ & $7,6 \pm 3,5$ & $5 \pm 4$ & $5,9 \pm 5,7$ & $7,6 \pm 4,2$ & $13,4 \pm 5$ \\
\hline NP & $-0,8 \pm 3,7$ & $-1,9 \pm 3,5$ & $0,6 \pm 4$ & $-7,7 \pm 5,7$ & $-1,6 \pm 4,2$ & $-5,3 \pm 5$ \\
\hline \multicolumn{7}{|c|}{$\mathrm{DDVI}^{\mathrm{a}}$} \\
\hline PP & $0,8 \pm 2$ & $1,3 \pm 2,3$ & $-1,1 \pm 2$ & $-0,1 \pm 1,8$ & $-0,5 \pm 1,7$ & $0,7 \pm 3$ \\
\hline NP & $-0,1 \pm 2$ & $-1,5 \pm 2,3$ & $-4,1 \pm 2$ & $-3 \pm 1,8$ & $-3,3 \pm 1,7$ & $-4,3 \pm 3$ \\
\hline \multicolumn{7}{|c|}{$\mathrm{DSVI}^{\mathrm{a}}$} \\
\hline $\mathrm{PP}$ & $-3 \pm 2,2$ & $-2,5 \pm 2,5$ & $15 \pm 4,7$ & $-10,5 \pm 2,6$ & $-13,3 \pm 2,6$ & $-10 \pm 3$ \\
\hline NP & $-3 \pm 2,2$ & $-3,7 \pm 2,8$ & $-4,8 \pm 4,7$ & $-2,4 \pm 2,6$ & $-1,9 \pm 2,6$ & $-3,1 \pm 3$ \\
\hline \multicolumn{7}{|c|}{ PPD } \\
\hline $\mathrm{PP}$ & $-2,6 \pm 4,5$ & $-9,5 \pm 4,8$ & $-4,6 \pm 5,1$ & $-0,6 \pm 5,6$ & $15,5 \pm 12$ & $9,5 \pm 10$ \\
\hline $\mathrm{NP}$ & $7,9 \pm 4,5$ & $-7,5 \pm 4,8$ & $6,1 \pm 5,1$ & $-7,8 \pm 5,6$ & $3,4 \pm 12,7$ & $5,3 \pm 10$ \\
\hline
\end{tabular}

TIVD = Tabique interventricular en diástole; TIVS = Tabique interventricular en sístole;

DDVI = Diámetro diastólico de VI; DSVI = Diámetro sistólico de VI; PPD = Pared posterior en diástole.

El superíndice $\left({ }^{a}\right)$ representa diferencias significativas $(p<0,05)$ entre grupos. 
Tabla 2: Porcentaje de cambio de parámetros ecocardiográficos (media $\pm E E$ ) de las mismas perras de la Tabla 1.

\begin{tabular}{|c|c|c|c|c|c|c|}
\hline \multicolumn{7}{|c|}{ Día de gestación } \\
\hline & 10 & 20 & 30 & 40 & 50 & 60 \\
\hline \multicolumn{7}{|c|}{ MV } \\
\hline PP & $6,5 \pm 5$ & $6,8 \pm 6$ & $3,1 \pm 7$ & $3,9 \pm 5$ & $11,7 \pm 8$ & $11 \pm 10$ \\
\hline NP & $5,6 \pm 5$ & $-7,8 \pm 6$ & $-2,4 \pm 7$ & $-18,5 \pm 5$ & $-0,7 \pm 8$ & $-15 \pm 10$ \\
\hline \multicolumn{7}{|c|}{ RRE } \\
\hline PP & $-3 \pm 5$ & $-8,4 \pm 5$ & $-2 \pm 6$ & $4,8 \pm 7$ & $7,9 \pm 7$ & $-1,6 \pm 7$ \\
\hline NP & $8,8 \pm 5$ & $-5,4 \pm 5$ & $10,9 \pm 6$ & $-5,4 \pm 7$ & $7,3 \pm 7$ & $11,7 \pm 7$ \\
\hline \multicolumn{7}{|c|}{ LA/Ao } \\
\hline PP & $-1,5 \pm 3$ & $0,5 \pm 4$ & $1,4 \pm 3$ & $5,8 \pm 4$ & $3,1 \pm 3$ & $-0,4 \pm 5$ \\
\hline NP & $3,7 \pm 3$ & $3,4 \pm 4$ & $-0,9 \pm 3$ & $4,3 \pm 4$ & $-3 \pm 3$ & $2 \pm 5$ \\
\hline \multicolumn{7}{|l|}{$\mathrm{PE}$} \\
\hline PP & $3,6 \pm 2$ & $2,2 \pm 2$ & $4,3 \pm 2$ & $1,1 \pm 2$ & $-2,2 \pm 2$ & $-2,4 \pm 2$ \\
\hline NP & $3,9 \pm 2$ & $2,4 \pm 2$ & $3,5 \pm 2$ & $0,1 \pm 2$ & $-2,1 \pm 2$ & $1,1 \pm 2$ \\
\hline \multicolumn{7}{|l|}{ AA } \\
\hline PP & $1,9 \pm 4$ & $5,5 \pm 4$ & $-2,3 \pm 4$ & $-6,3 \pm 5$ & $-4,2 \pm 5$ & $2,2 \pm 6$ \\
\hline NP & $-2,2 \pm 4$ & $-4,6 \pm 4$ & $-1,3 \pm 4$ & $-7,2 \pm 5$ & $-3,8 \pm 5$ & $3 \pm 6$ \\
\hline \multicolumn{7}{|c|}{ PSSE } \\
\hline PP & $8,9 \pm 8$ & $15 \pm 16$ & $3 \pm 8$ & $3 \pm 12$ & $3,5 \pm 8$ & $7,4 \pm 12$ \\
\hline NP & $-6,3 \pm 8$ & $-7,6 \pm 16$ & $16,4 \pm 8$ & $-6,1 \pm 12$ & $-4,3 \pm 8$ & $-3 \pm 12$ \\
\hline
\end{tabular}

$\mathrm{MV}=$ Masa ventricular; $\mathrm{RRE}=$ Relación radio espesor; $\% \mathrm{MAC}=$ Porcentaje medio de acortamiento circunferencial; $\mathrm{Ao} / \mathrm{AI}=$ Relación $\mathrm{Ao} / \mathrm{AI} ; \mathrm{PE}=$ Período eyectivo; $\mathrm{AA}=$ Amplitud aórtica; PSSE = Punto de separación septal E. 


\section{Discusión}

La mayor parte de nuestros resultados estuvieron en línea con aquellos reportados en humanos, especie en la que el embarazo se asocia con una creciente sobrecarga cardíaca materna (5). En el presente estudio, el engrosamiento de las paredes cardíacas demuestra que, como en la mujer (4), el corazón desarrolla una hipertrofia compensatoria durante la preñez canina, probablemente en respuesta a la sobrecarga de volumen (13). Más aún, el DDVI fue mayor en el grupo PP, lo que coincide con reportes previos en mamíferos que indican que esta hipertrofia es de tipo excéntrica (2).

La hipertrofia cardíaca es un mecanismo fisiológico que intenta mantener el gasto cardíaco a pesar de la sobrecarga crónica creciente sobre el VI. El estímulo más importante para el desarrollo de este cambio morfológico es el estiramiento de las fibras miocárdicas (2). En ratones, la hipertrofia cardíaca de la preñez se distingue de la hipertrofia patológica por la ausencia de los marcadores moleculares que caracterizan a esta última. En esta especie, la expresión de estos marcadores es bloqueada por el estrógeno (2). Algunos aspectos aún no determinados de la preñez, posiblemente hormonales, podrían ser responsables de las alteraciones en las propiedades intrínsecas del miocardio materno, independientemente de los cambios hemodinámicos asociados (7). De ser correcto esto, el tratamiento hormonal podría llegar a mejorar la función cardíaca en casos de falla miocárdica.

En contraste con lo descripto para la mujer, en donde los resultados para \% MAC y FA son contradictorios (6-8), ambos parámetros fueron significativamente más altos en el grupo PP que en el NP, a partir del día 30. Esto también difiere con trabajos previos en perras, que mostraron ausencia de cambios o incluso una disminución en la función sistólica en la preñez tardía $(13,15)$. Estos hallazgos podrían relacionarse con una mejora en la función sistólica 
muy probablemente debida al aumento de la volemia durante la gestación y al engrosamiento de las paredes miocárdicas. También es posible que el descenso de la PAS haya influenciado el incremento de la FA y de la función sistólica.

Considerando la relevancia de la predisposición racial en la aparición de enfermedad miocárdica (22), sería interesante evaluar si este aumento en la eficiencia sistólica varía según el tamaño y raza de los perros.

El incremento de la distensibilidad vascular y, posiblemente, la presencia del lecho vascular uteroplacentario, resultan en un aumento de la compliance vascular. Como se ha descripto anteriormente en perros $(16,17)$, el crecimiento de las membranas fetales y los capilares placentarios pueden inducir una caída significativa del IR de la arteria uterina. Esta disminución está asociada a un descenso de la resistencia vascular total (1), en tanto que ambos decrecen desde el día 30 de preñez. Esto también podría reflejar la influencia hormonal en la circulación periférica, debido por ejemplo, al aumento en la disponibilidad de óxido nítrico que se produce en la gestación (6). Más aún, la actividad refleja de los barorreceptores en la hipotensión inducida parece estar alterada durante la preñez canina (14). El descenso en este parámetro contrasta un reporte previo en perras de raza San Bernardo (15). Sería interesante probar si este descenso se relaciona o no con el peso y la raza, y si se observa también en casos de gestaciones patológicas.

Debido a que la poscarga se refleja en la resistencia periférica, la caída de esta última es el principal determinante del aumento en el VS y GC (34), fenómeno que garantiza una adecuada irrigación del conceptus. En este estudio, el VS y GC crecieron desde el día 40 de gestación, 10 días antes que lo reportado previamente (13). El aumento de GC podría también deberse al alza en la FC materna, en el VS y en la precarga. Si bien durante la preñez canina 
se desencadena un estado de anemia $(35,36)$, ésta resulta insuficiente para provocar las modificaciones encontradas en el sistema cardiovascular materno.

En conclusión, la función cardíaca y la circulación periférica maternas se modifican a lo largo de la preñez en caninos. El descenso en la poscarga, el incremento en el GC y la hipertrofia cardíaca resultan de los cambios hemodinámicos que ocurren durante la gestación canina. Por lo tanto, la adaptación cardíaca materna en las perras juega un papel vital en el mantenimiento de la perfusión uterina óptima para el desarrollo fetal. La evaluación de la función cardiovascular materna podría convertirse en una herramienta sumamente útil en la detección de complicaciones obstétricas en perros.

\section{Bibliografía}

1. Valensise H, Novelli GP, Vasapollo B, Borzi M, Arduini D, Galante A, Romanini C. Maternal cardiac systolic and diastolic function: relationship with uteroplacental resistances. A Doppler and echocardiographic longitudinal study. Ultrasound Obstet Gynecol 2000;15:487-97.

2. Eghbali M, Deva R, Alioua A, Minosyan TY, Ruan H, Wang Y, Toro L, Stefani E. Molecular and Functional Signature of Heart Hypertrophy During Pregnancy. Circ Res 2005;96:1208-16.

3. Blanco PG, Arias DO, Gobello C. Doppler ultrasound in canine pregnancy. J Ultrasound Med 2008;27:1745-50. 
4. Kametas NA, McAuliffe F, Hancock J, Chambers J, Nicolaides KH. Maternal left ventricular mass and diastolic function during pregnancy. Ultrasound Obstet Gynecol 2001a;18:460-6.

5. Desai D, Moodley J, Naidoo DP. Echocardiographic assessment of cardiovascular hemodynamics in normal pregnancy. Obstet Gynecol 2004;104:20-9.

6. Simmons L, Gillin AG, Jeremy RW. Structural and functional changes in left ventricle during normotensive and preeclamptic pregnancy. Am J Physiol HeartCirc Physiol 2002;283:H1627-33.

7. Mone SM, Sanders SP, Colan SD. Control mechanisms for physiological hypertrophy of pregnancy. Circulation 1996;94:667-72.

8. Kametas NA, McAuliffe F, Cook B, Nicolaides KH, Chambers J. Maternal left ventricular transverse and long-axis systolic function during pregnancy. Ultrasound Obstet Gynecol $2001 b ; 18: 467-74$.

9. Easterling TR, Watts DH, Schmucker BC, Benedetti TJ. Measurement of cardiac output during pregnancy: validation of Doppler technique and clinical observations in preeclampsia. Obstet Gynecol 1987;69:845-50.

10. Vasapollo B, Novelli GP, Valensise H. Total vascular resistance and left ventricular morphology as screening tools for complications in pregnancy. Hypertension 2008;51: 10206.

11. Khaw A, Kametas NA, Turan OM, Bamfo JE, Nicolaides KH. Maternal cardiac function and uterine artery Doppler at 11-14 weeks in the prediction of pre-eclampsia in nulliparous women. BJOG - Int J Obstet Gyn 2008;115:369-76.

12. Voss A, Baumert M, Baier V, Stepan H, Walther T, Faber R. Autonomic cardiovascular control in pregnancies with abnormal uterine perfusion. Am J Hypertens 2006;19:306-12. 
13. Williams JG, Rincon-Skinner T, Sun D, Wang Z, Zhang S, Zhang X, Hintze TH. Role of nitric oxide in the coupling of myocardial oxygen consumption and coronary vascular dynamics during pregnancy in the dog. Am J Physiol Heart Circ Physiol 2007;293:H2479-86. 14. Brooks VL, Keil LC. Changes in the baroreflex during pregnancy in conscious dogs: Heart rate and hormonal responses. Endocrinology 1994;135:1894-901.

15. Lobo L, Salazar L, Oliveira P, Pereira A, Fontes-Sousa AP. Echocardiographic evaluation of maternal cardiac function in the Saint Bernard dog. J Vet Int Med 2009;23:1319-50.

16. Nautrup CP. Doppler ultrasonography of canine maternal and fetal arteries during normal gestation. Journal of Reproduction and Fertility 1998;112:301-314.

17. Di Salvo P, Bocci F, Polisca A. Doppler evaluation of maternaland fetal vessels during normal gestation in the bitch.Res Vet Sci 2006;81:382-8.

18. England GCW. Ultrasonograpic assessment of abnormal pregnancy. Ultrasonography 1998;28:849-67.

19. Olson PN, Husted PW, Allen TA, Nett TM. Reproductive endocrinology and physiology of the bitch and queen. Vet Clin North Am Small Anim Pract 1984;14:927-46.

20. Chetboul V, Tidholm A, Nicolle A, Sampedrano CC, Gouni V, Pouchelon JL, Lefebvre HP, Concordet D. Effects of animal position and number of repeated measurements on selected two-dimensional and M-mode echocardiographic variables in healthy dogs. J Am Vet Med A 2005;227:743-7.

21. Thomas WP, Gaber CE, Jacobs GJ, Kaplan PM, Lombard CW, Moise NS, Moses BL. Recommendations for standards in transthoracic two-dimensional echocardiography in the dog and cat. Echocardiography Committee of the Specialty of Cardiology, American College of Veterinary Internal Medicine. J Vet Intern Med 1993;7:247-52. 
22. Kittleson MD. Primary myocardial disease.En: Kittelson MD, Kienle RD, editores. Small animal cardiovascular medicine textbook. St. Louis, Mosby; 1998. p. 319-46.

23. Boon J. The echocardiographyc examination. En: Boon JA, editora. Manual of Veterinary Echocardiography. Media, Williams and Wilkins; 1998. p. 35-128.

24. Kienle RD. Echocardiography. En: Kittelson MD, Kienle RD, editores. Small animal cardiovascular medicine textbook. St. Louis, Mosby; 1998. p. 95-117.

25. Vuille C, Weyman AE. Left ventricle I: general considerations, assessment of chamber size and function. En: Weyman AE, editor. Principles and practice of echocardiography. Media, Williams and Wilkins;1994. p. 575-624.

26. Estrada A, Maisenbacher H. Calculation of stenotic valve area. J Vet Cardiol 2006;8:4953.

27. Gottdiener JS, Bednarz J, Devereux R, Gardin J, Klein A, Manning WJ, Morehead A, Kitzman D, Oh J, Quinones M, Schiller NB, Stein JH, Weissman NJ. American Society of Echocardiography recommendations for use of echocardiography in clinical trials. J Am Soc Echocardiog 2004;17:1086-119.

28. Littman MP, Fox PR. Systemic hypertension: recognition and treatment. En: Fox P, Sisson D, Moïse NS, editores. Textbook of canine and feline cardiology. Philadelphia, WB Saunders Co;1999. p. 795-813.

29. Alvarez-Clau A, Liste F. Ultrasonographic characterization of the uterine artery in the nonestrus bitch. UltrasoundMed Biol 2005;3:1583-7.

30. Dickey RP. Doppler ultrasound investigation of uterine and ovarian blood flow in infertility and early pregnancy. Hum Reprod Update 1997;3:467-503. 
31. Cornell CC, Kittleson MD, Della Torre P, Häggström J, Lombard CW, Pedersen HD, Vollmar A, Wey A. Allometric scaling of M-mode cardiac measurements in normal adult dogs. J Vet Inter Med 2004;18:311-21.

32. Borgarelli M, Tarducci A, Zanatta R, Haggstrom J. Decreased systolic function and inadequate hypertrophy in large and small breed dogs with chronic mitral valve insufficiency. J Vet Inter Med 2007;21:61-7.

33. Schiller NB, Skiôldebrand CG, Schiller EJ, Mavroudis CC, Silverman NH, Rahimtoola SH, Lipton MJ. Canine left ventricular mass estimation by two-dimensional echocardiography. Circulation 1983;68:210-6.

34. Spotswood TC, Kirberger RM, Koma LM, Thompson PN, Miller DB. Changes in echocardiographic variables of left ventricular size and function in a model of canine normovolemic anemia. Vet RadiolUltrasound 2006;47:358-65.

35. Concannon PW. Reproductive physiology and endocrinology patterns of the bitch. En: Kirk RW, editor. Current Veterinary Therapy VIII. Philadelphia, W.B. Saunders Co;1983, pp. 886-901.

36. Johnson CA. Pregnancy management in the bitch. Theriogenology 2008;70:1412-17. 


\section{CAPÍTULO II}

\section{UN MODELO EXPERIMENTAL PARA EL ESTUDIO CARDIOVASCULAR MATERNO - FETAL DEL DESENLACE GESTACIONAL ADVERSO EN CANINOS}

\section{Introducción}

La adaptación cardiovascular materna es esencial para garantizar una adecuada irrigación y desarrollo fetal. La ultrasonografía Doppler y la ecocardiografía son métodos de diagnóstico no invasivos y confiables para evaluar el sistema cardiovascular materno y fetal (1-3).

La evaluación Doppler de las arterias maternas y fetales permite conocer el nivel de perfusión del conceptus (4). Con este método, se registra la velocidad, el gradiente de presión (GP) y el índice de resistencia (IR) de cada vaso arterial. El IR de las arterias se considera un indicador del desarrollo de los componentes maternos y fetales, que es proporcional a la resistencia que presenta el flujo sanguíneo distal al sitio de medición. Por lo tanto, a medida que la preñez progresa, el IR decrece debido al crecimiento de la vasculatura placentaria y fetal $(2,3,5)$. En mujeres embarazadas, un ascenso del IR de la arteria uterina y de otros vasos fetales es indicativo de un riesgo elevado de afecciones obstétricas tales como restricción de crecimiento del conceptus, alta probabilidad de muerte fetal o perinatal (6-8).

En caninos domésticos, la perfusión uteroplacentaria ha sido descripta durante la preñez normal, conjuntamente con el flujo de la arteria umbilical, aorta fetal y carótida común fetal $(2,3,9)$. 
La ecocardiografía, por su parte, permite monitorear los cambios en la morfología y el funcionamiento cardíaco materno. Durante la gestación canina normal, tienen lugar una serie de cambios hemodinámicos, que consisten en un aumento de la función sistólica, del gasto cardíaco (GC) y una disminución en la poscarga (10). En obstetricia humana, la ausencia de estos mecanismos adaptativos del aparato cardiovascular, se relaciona con desenlaces gestacionales adversos (11-13). Existe evidencia de que las gestaciones complicadas con restricción de crecimiento intrauterino se asocian con una inadecuada expansión del espacio intravascular materno y una falta de incremento del gasto cardíaco (1).

Sin embargo, no existen hasta el momento reportes sobre el comportamiento de los parámetros cardiovasculares maternos y fetales durante la gestación canina patológica. Por todo esto, el objetivo de este estudio fue describir los cambios en la morfología cardíaca, función sistólica y circulación materna y fetal en un modelo experimental de gestación anormal farmacológicamente inducida.

\section{Materiales y métodos}

Animales y seguimiento

Dieciséis perras preñadas (30 a 35 días del primer servicio) y saludables, de 6 meses a 6 años de edad, de 3 a $40 \mathrm{~kg}$ de peso, mestizas y de razas puras, se asignaron al azar a uno de los siguientes grupos de tratamiento. El grupo de hembras medicadas (GM; n=10) recibió un tratamiento de cabergolina y cloprostenol para interrumpir la preñez (14). El grupo control $(\mathrm{CO} ; \mathrm{n}=6)$ estuvo conformado por hembras preñadas que no recibieron ningún tratamiento. 
Cada uno de los grupos se evaluó a partir del día de la consulta (30 a 35 días del primer servicio), y cada 3 días hasta cumplir con tres observaciones. Este estudio fue aprobado por el Comité de Cuidado y Uso Animal de esta Institución.

\section{Evaluaciones ecocardiográficas}

Las evaluaciones ecocardiográficas bidimensionales, en modo M y Doppler se realizaron con los animales en estación, utilizando un transductor $7 \mathrm{MHz}$ (Toshiba Core Vision Pro; 15). Sobre la piel rasurada, se aplicó gel para lograr un adecuado acomplamiento acústico. Las ventanas paraesternales utilizadas fueron las previamente descriptas por Thomas y col. (1996; 16).

Se registraron las medidas del diámetro diastólico del VI (DDVI), diámetro sistólico del VI (DSVI), tabique interventricular en diástole (TIVD) y sístole (TIVS), pared posterior del VI en diástole (PPD) y sístole (PPS) y fracción de acortamiento (FA), por medio de modo M (17). La FA se calculó como (DDVI-DSVI)/DDVI x 100 (18). La masa ventricular (MV), relación radio espesor (RRE), estrés de fin de sístole (EFS) y porcentaje medio de acortamiento circunferencial (\%MAC) se calcularon de acuerdo a las fórmulas correspondientes (17-19). Con el mismo modo ecocardiográfico, se registraron la relación Ao/AI (Ao/AI), período eyectivo (PE), amplitud aórtica (AA) y punto de separación septal E (PSSE; 17).

Con ecocardiografía Doppler espectral se registró el VTI del flujo transaórtico a partir del cual se calcularon el volumen sistólico (VS) y el GC (18,20). Para minimizar la variabilidad en las mediciones, todas fueron efectuadas por un único operador entrenado y siguiendo guías de recomendación específicas $(16,21)$. Se promediaron tres valores de cada parámetro. 


\section{Presión arterial sistémica}

La presión arterial sistémica (PAS) se midió utilizando un equipo Doppler vascular (DV610 Medmega). Se utilizaron manguitos cuyo ancho correspondió al 40\% de la circunferencia total del miembro anterior (22). Los animales se colocaron en decúbito lateral y se promediaron tres registros.

Evaluación ultrasonográfica del flujo sanguíneo materno y fetal

El diagnóstico gestacional, estimación del tamaño de camada, muerte fetal e interrupción de la preñez se realizaron por medio de ultrasonografía bidimensional (Toshiba Core Vision Pro, Japón) con un transductor lineal de 5-8 MHz $(23,24)$. Las perras se colocaron en decúbito lateral, se rasuró la piel del vientre y se aplicó gel para lograr un adecuado acomplamiento acústico. La ultrasonografía bidimensional se utilizó para identificar un corte transversal del cuerpo uterino. Luego, se utilizó Doppler color para localizar ambas arterias uterinas y Doppler pulsado para obtener los espectros (25). Para minimizar la variabilidad, se registraron tres ondas uniformes y consecutivas por un único operador entrenado. Se midieron la velocidad de pico sistólico (VMAX) y velocidad de flujo de fin de diástole (VMIN). El IR [(VMAX - VMIN)/VMAX] y el GP se calcularon automáticamente (5).

En ambos grupos se realizó una ecocardiografía en modo M y Doppler en el feto más caudal del cuerno uterino derecho. La arteria umbilical y aorta abdominal fetal se evaluaron con Doppler color y pulsado según reportes previos (3). Se registraron tres ondas uniformes y consecutivas de cada arteria para medir VMAX y VMIN. El IR y GP se calcularon 
automáticamente. La FC fetal ser registró con ecocardiografía en modo M y se promediaron tres mediciones (29).

\section{Electrocardiogramas}

Para realizar el electrocardiograma (ECG), los pacientes se posicionaron en decúbito lateral derecho sobre una camilla cubierta de goma. Las perras se sujetaron suavemente con los cuatro miembros sostenidos perpendiculares al cuerpo y apenas separados (30). Los electrodos se acoplaron a la piel con clips tipo cocodrilos metálicos que se humedecieron con alcohol para mejorar la transmisión eléctrica.

Las derivaciones I, II, III, $\mathrm{aV}_{\mathrm{R}}, \mathrm{aV}_{\mathrm{L}}, \mathrm{aV}_{\mathrm{F}}, \mathrm{CV}_{6 \mathrm{LU}}, \mathrm{CV}_{6 \mathrm{LL}}, \mathrm{CV}_{5 \mathrm{RL}}$ y $\mathrm{V}_{10}$ se registraron en papel calibrado a una velocidad de $50 \mathrm{~mm} / \mathrm{s}$, como ha sido previamente reportado (31). La derivación II se registró también a una velocidad de $25 \mathrm{~mm} / \mathrm{s}$ para calcular la FC y ritmo cardíaco (RC; ritmo sinusal o arritmia sinusal). Luego del registro, un único operador entrenado se encargó de analizar cada ECG. El eje eléctrico medio (EEM), amplitud de onda P (Pa), duración de onda P (Pd), intervalo P-R (PR), amplitud del complejo QRS (QRSa), duración del complejo QRS (QRSd), intervalo Q-T (QT), segmento S-T (ST; mv) y onda T ( $\mathrm{T}$; menor o mayor al 25\% de amplitud de la onda $\mathrm{R}$ ) se calcularon manualmente a una velocidad de papel de $50 \mathrm{~mm} / \mathrm{s}$ (32). Se promediaron tres latidos y seis latidos en caso de arritmia sinusal. 


\section{Análisis estadístico}

Con el fin de constatar la comparabilidad de ambos grupos en función de la edad, peso, parición y tamaño de camada, se efectuó un test de Student. Los IR de las arterias uterina derecha e izquierda fueron comparados en ambos grupos utilizando el mismo test. Todas las variables continuas a excepción del ST, fueron transformadas a porcentaje de cambio [(Valor final - valor inicial / valor inicial) x 100] y los grupos se compararon con ANOVA de medidas repetidas seguido de un test de Tukey. Las variables categóricas se analizaron con un test de Chi cuadrado (SPSS 17.0, SPSS Inc.).

\section{Resultados}

Durante todo el período de evaluación, no se registraron diferencias significativas entre los valores Doppler de la arteria uterina derecha e izquierda $(\mathrm{p}>0,1)$, motivo por el cual los resultados se calcularon utilizando una de ambas arterias. Además, todos los valores previos al tratamiento no difirieron entre GM y CO $(\mathrm{p}>0,1)$. Ambos grupos de pacientes resultaron similares en cuanto a su peso, edad, parición y tamaño de camada $(\mathrm{p}>0.1)$. La totalidad de las perras del GM (10/10) y ninguna del CO (0/6) interrumpieron la gestación. En las perras tratadas, el aborto se produjo a los 6 $\pm 1,2$ (media $\pm E E$ ) días de ser medicadas, con un rango de 2 a 12 días.

Luego de aplicado el tratamiento, se registró una interacción significativa entre el tiempo y el grupo para los porcentajes de cambio de la PAS $(\mathrm{p}<0,05)$, VMAX $(\mathrm{p}<0,05), \operatorname{VMIN}(\mathrm{p}<$ 
$0,01)$ y el IR $(\mathrm{p}<0,01)$ de la arteria uterina, IR de la arteria umbilical $(\mathrm{p}<0,01)$ y el IR de la aorta abdominal $(\mathrm{p}<0,01)$.

La PAS presentó un descenso en el CO, mientras que en el GM se incrementó este valor, difiriendo ambos grupos a partir del tercer día de tratamiento (Figura 1). El grupo de hembras tratadas presentó valores inferiores en VMAX en la arteria uterina a partir del sexto día de tratamiento (Figura 2), mientras que la VMIN fue menor desde el tercer día (Figura 3). A partir de ese mismo día, el IR de la arteria uterina fue mayor en el GM (Figura 4).

Por otro lado, al día 6 del estudio, el IR de la arteria umbilical disminuyó más en el CO que en el GM (Figura 5). Al mismo día, el IR de la aorta abdominal fetal desarrolló valores más bajos en el CO que en el GM (Figura 6).

El porcentaje de cambio del PE mostró valores más bajos en el GM ( $p<0,05$; Tabla 1). El porcentaje de cambio del EFS presentó valores mayores en el GM que en el $\mathrm{CO}$ ( $\mathrm{p}<0,05$;

Tabla 1).

El GP de la arteria uterina, fue mayor en el CO que en el GM ( $<<0,05$; Tabla 1).

El TIVD y TIVS, DDVI y DSVI, PPD y PPS, FA, MV, RRE, \% MAC, Ao/AI, AA, PSSE, VS, GC, VMAX, VMIN y GP de la arteria umbilical, VMAX, VMIN y GP de la aorta abdominal fetal y FC fetal no difirieron entre los grupos (Tabla 1). Tampoco lo hicieron los parámetros electrocardiográficos maternos evaluados (Tablas 2 y 3). 


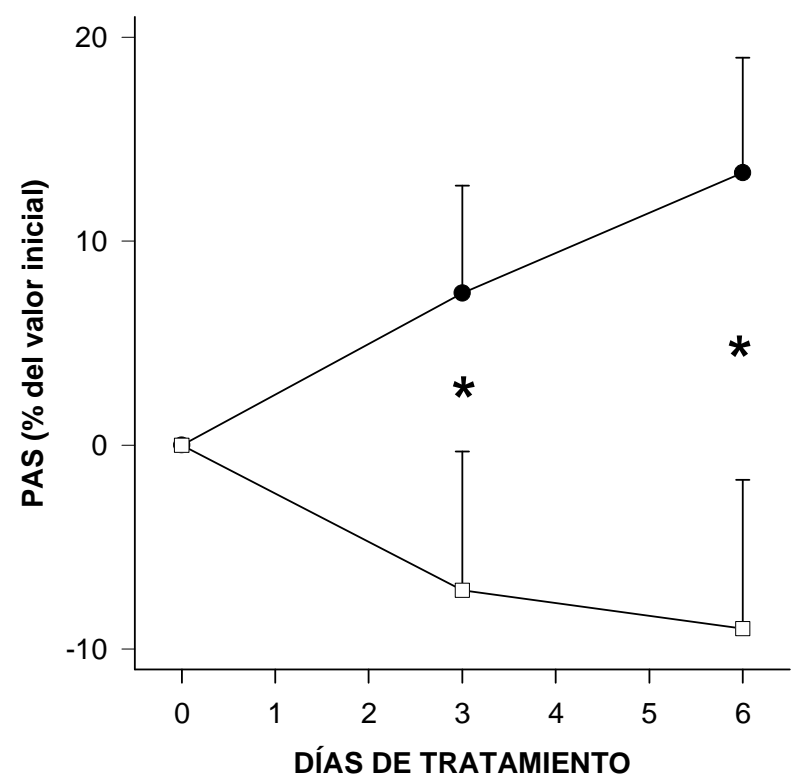

Figura 1. Porcentaje de cambio de la presión arterial sistémica (PAS; media $\pm E E)$ de 10 perras gestantes medicadas (GM; círculos) y 6 perras gestantes control (CO; cuadrados) evaluadas cada tres días desde el inicio del tratamiento.

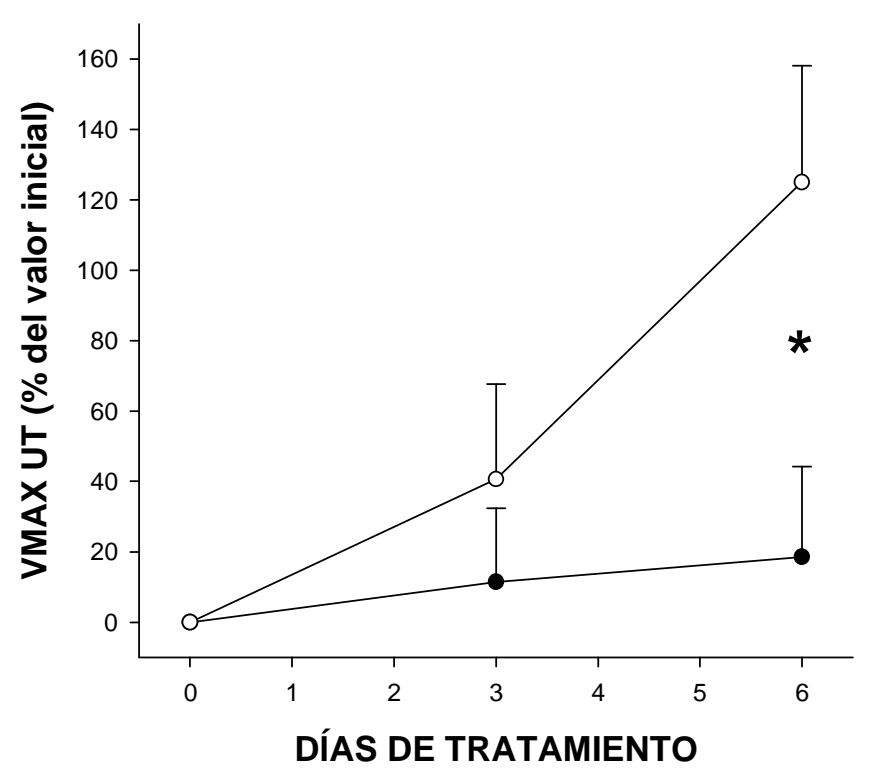

Figura 2. Porcentaje de cambio de la velocidad de pico sistólico de la arteria uterina (VMAX UT; media $\pm E E)$ de las mismas perras de la Figura 1. 


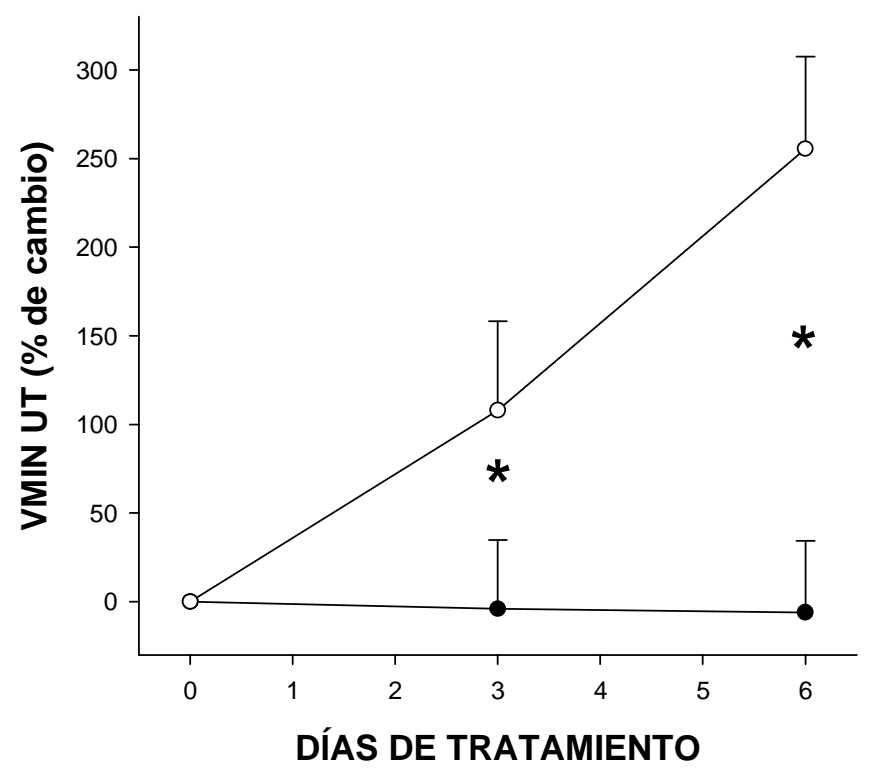

Figura 3. Porcentaje de cambio de la velocidad de flujo de fin de diástole de la arteria uterina (VMIN UT; media $\pm E E$ ) de las mismas perras de la Figura 1.

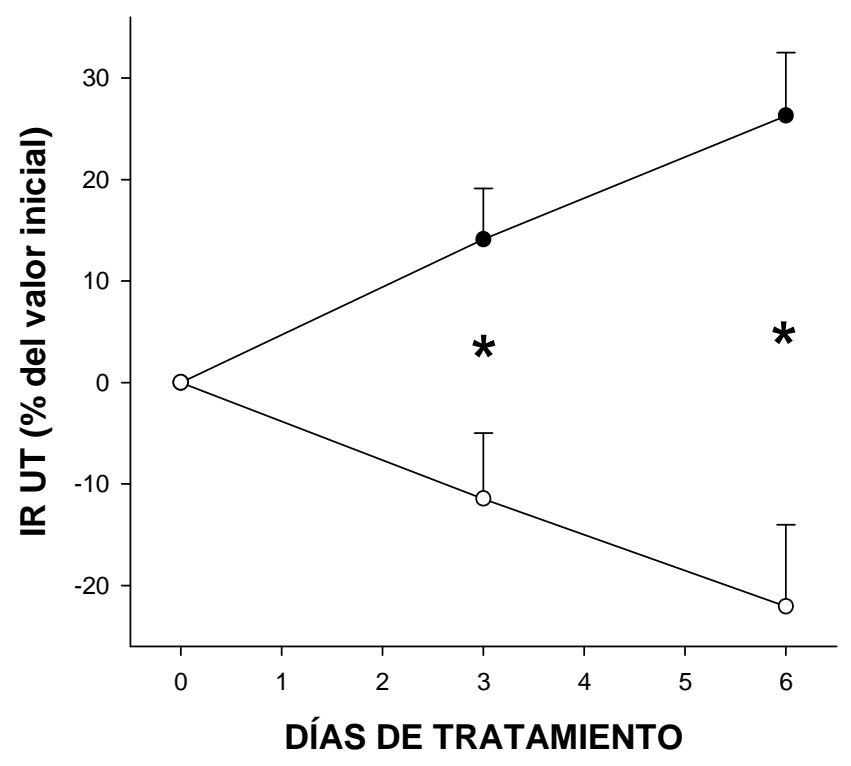

Figura 4. Porcentaje de cambio del índice de resistencia de la arteria uterina (IR UT; media \pm EE) de las mismas perras de la Figura 1. 


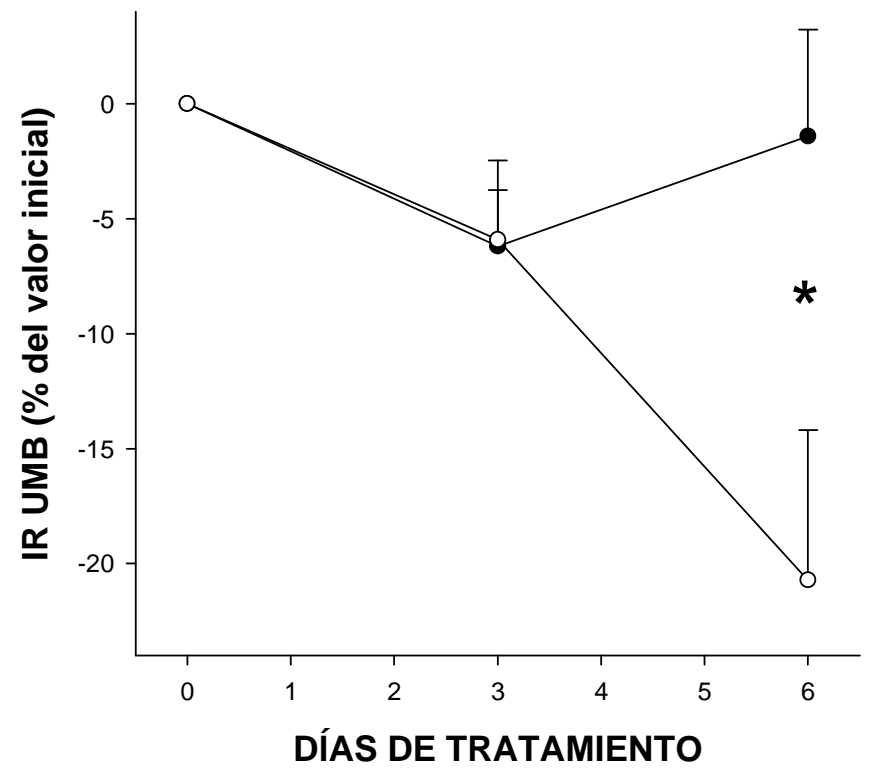

Figura 5. Porcentaje de cambio del índice de resistencia de la arteria umbilical (IR UMB; media $\pm \mathrm{EE})$ de las mismas perras de la Figura 1.

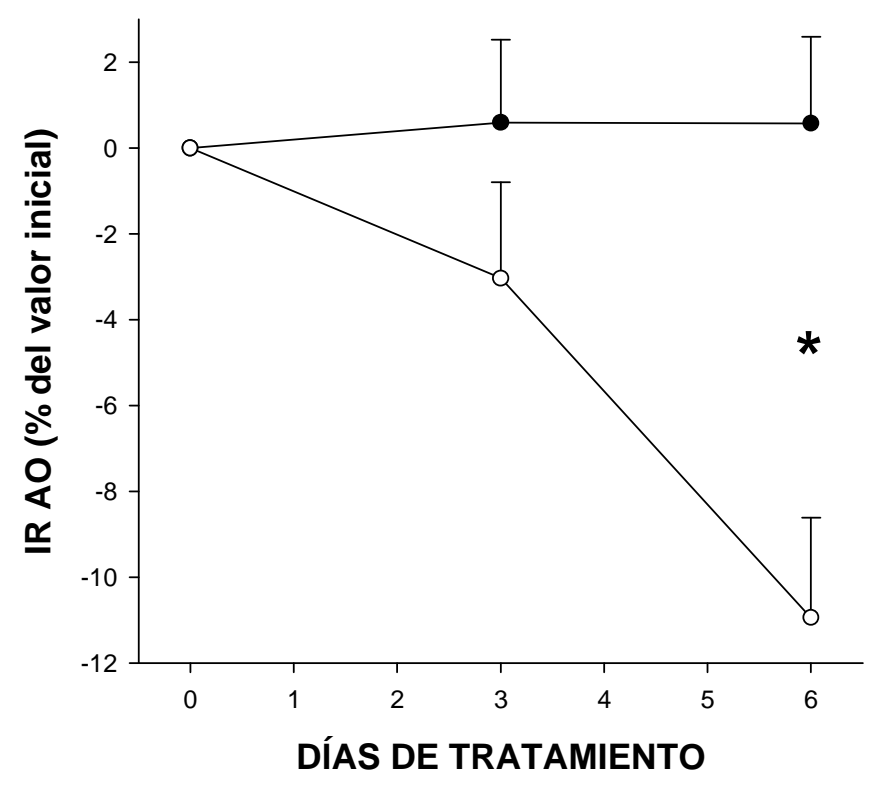

Figura 6. Porcentaje de cambio del índice de resistencia de la aorta abdominal fetal (IR AO; media $\pm E E)$ de las mismas perras de la Figura 1. 
Tabla 1. Porcentaje de cambio (media $\pm E E$ ) de parámetros ecográficos de 10 perras gestantes medicadas (GM) y 6 perras gestantes control (CO) evaluadas cada tres días desde el inicio del tratamiento.

\begin{tabular}{|c|c|c|c|c|}
\hline \multirow{3}{*}{ Parámetro } & \multicolumn{4}{|c|}{ Días de tratamiento } \\
\hline & \multicolumn{2}{|c|}{ Día 3} & \multicolumn{2}{|c|}{ Día 6} \\
\hline & GM & $\mathrm{CO}$ & GM & $\mathrm{CO}$ \\
\hline$P E^{a}$ & $-6,1 \pm 1$ & $0,7 \pm 2$ & $-4,6 \pm 1$ & $1,5 \pm 2$ \\
\hline EFS $^{\mathrm{a}}$ & $21,1 \pm 12$ & $-8,3 \pm 15$ & $19,0 \pm 11$ & $-21,5 \pm 14$ \\
\hline GP UT $^{a}$ & $25,5 \pm 149$ & $412 \pm 193$ & $27,7 \pm 61$ & $312 \pm 79$ \\
\hline TIVD & $-6,3 \pm 5$ & $3,2 \pm 6$ & $-3,6 \pm 5$ & $3,6 \pm 7$ \\
\hline TIVS & $4,8 \pm 3$ & $1,9 \pm 4$ & $0,0 \pm 3$ & $-6,2 \pm 4$ \\
\hline DDVI & $-2,0 \pm 3$ & $0,4 \pm 4$ & $-2,1 \pm 2$ & $-6,5 \pm 3$ \\
\hline DSVI & $1,9 \pm 5$ & $-0,1 \pm 6$ & $3,8 \pm 5$ & $-6,8 \pm 7$ \\
\hline PPD & $-3,2 \pm 5$ & $2,8 \pm 7$ & $7,1 \pm 5$ & $12,8 \pm 7$ \\
\hline PPS & $-5,0 \pm 3$ & $3,1 \pm 4$ & $1,8 \pm 6$ & $12,9 \pm 8$ \\
\hline FA & $3,8 \pm 4$ & $1,0 \pm 6$ & $-3,2 \pm 4$ & $1,8 \pm 6$ \\
\hline MV & $-11,2 \pm 5$ & $1,5 \pm 7$ & $218 \pm 179$ & $-8,6 \pm 231$ \\
\hline RRE & $0,3 \pm 7$ & $3,3 \pm 9$ & $99,9 \pm 72$ & $23,1 \pm 93$ \\
\hline$\% \mathrm{MAC}$ & $6,2 \pm 6$ & $-3,4 \pm 8$ & $-0,8 \pm 6$ & $3,0 \pm 9$ \\
\hline $\mathrm{Ao} / \mathrm{AI}$ & $-3,9 \pm 4$ & $-8,7 \pm 5$ & $-1,6 \pm 5$ & $-4 \pm 6$ \\
\hline AA & $-1,6 \pm 4$ & $-1,1 \pm 6$ & $5,1 \pm 3$ & $-7,9 \pm 4$ \\
\hline PSSE & $7,1 \pm 10$ & $4,8 \pm 12$ & $26,2 \pm 8$ & $-4,8 \pm 10$ \\
\hline VS & $1,7 \pm 8$ & $-4,5 \pm 11$ & $2,5 \pm 6$ & $12,4 \pm 8$ \\
\hline GC & $5,5 \pm 11$ & $6,4 \pm 14$ & $8,7 \pm 8$ & $-7,9 \pm 10$ \\
\hline VMAX UMB & $29,5 \pm 50$ & $552 \pm 71$ & $9,9 \pm 71$ & $858 \pm 101$ \\
\hline VMIN UMB & $107 \pm 61$ & $138 \pm 79$ & $116 \pm 126$ & $511 \pm 163$ \\
\hline GP UMB & $137 \pm 157$ & $566 \pm 192$ & $70 \pm 120$ & $850 \pm 147$ \\
\hline VMAX AO & $25,0 \pm 26$ & $232 \pm 34$ & $17,1 \pm 54$ & $555 \pm 73$ \\
\hline VMIN AO & $-3,7 \pm 15$ & $20 \pm 20$ & $-16,8 \pm 34$ & $280 \pm 43$ \\
\hline GP AO & $106 \pm 103$ & $85,6 \pm 93$ & $60 \pm 139$ & $590 \pm 125$ \\
\hline FCF & $5,2 \pm 2$ & $5,9 \pm 2$ & $3,6 \pm 1$ & $2,4 \pm 2$ \\
\hline
\end{tabular}

$\mathrm{PE}=$ Período eyectivo $;$ EFS $=$ Estrés de fin de sístole $;$ GP UT = Gradiente de presión de la arteria uterina; TIVD = Tabique interventricular en diástole; TIVS = Tabique interventricular en sístole; DDVI = Diámetro diastólico de VI; DSVI = Diámetro sistólico de VI; PPD = Pared posterior en diástole; $\mathrm{PPS}=$ Pared posterior en sístole; FA = Fracción de acortamiento; $\mathrm{MV}=$ 
Masa ventricular; $\mathrm{RRE}=$ Relación radio espesor; $\% \mathrm{MAC}=$ Porcentaje medio de acortamiento circunferencial; $\mathrm{Ao} / \mathrm{AI}=$ Relación $\mathrm{Ao} / \mathrm{AI} ; \mathrm{AA}=$ Amplitud aórtica; $\mathrm{PSSE}=$ Punto de separación septal E; VS = Volumen sistólico; GC = Gasto cardíaco; VMAX UMB = Velocidad de pico sistólico de la arteria umbilical; VMIN UMB = Velocidad de flujo de fin de diástole de la arteria umbilical; GP UMB = Gradiente de presión de la arteria umbilical; VMAX AO = Velocidad de pico sistólico de la aorta abdominal fetal; VMIN AO = Velocidad de flujo de fin de diástole de la aorta abdominal fetal; GP AO = Gradiente de presión de la aorta abdominal fetal; FCF = Frecuencia cardíaca fetal El superíndice (a) representa diferencias significativas $(\mathrm{p}<0,05)$ entre grupos.

Tabla 2. Porcentaje de cambio (media $\pm E E$ ) de parámetros electrocardiográficos de las mismas perras de la Tabla 1.

\begin{tabular}{|c|c|c|c|c|}
\hline \multirow{2}{*}{ Parámetro } & \multicolumn{4}{|c|}{ Días de tratamiento } \\
\cline { 2 - 5 } & \multicolumn{2}{|c|}{ Día 3} & GM & CO \\
\cline { 2 - 5 } & GM & CO & $-6,8 \pm 9$ & $12,2 \pm 12$ \\
\hline FC & $0,9 \pm 5$ & $9,9 \pm 7$ & $5,3 \pm 40$ & $117 \pm 52$ \\
\hline EEM & $13,0 \pm 16$ & $37,5 \pm 21$ & $35 \pm 21$ & $16,6 \pm 27$ \\
\hline $\mathrm{Pa}$ & $26,6 \pm 21$ & $25 \pm 28$ & $-8 \pm 9$ & $8,3 \pm 11$ \\
\hline $\mathrm{Pd}$ & $2,5 \pm 2$ & $4,1 \pm 3$ & $51,8 \pm 58$ & $0,0 \pm 75$ \\
\hline $\mathrm{PR}$ & $60,7 \pm 57$ & $4,2 \pm 74$ & $-10,0 \pm 13$ & $-1,3 \pm 16$ \\
\hline QRSa & $16,8 \pm 10$ & $-8,8 \pm 13$ & $5,5 \pm 12$ & $11,1 \pm 16$ \\
\hline QRSd & $6,6 \pm 5$ & $0,0 \pm 7$ & $-5,2 \pm 8$ & $-4,4 \pm 11$ \\
\hline QT & $4,3 \pm 2$ & $0,0 \pm 3$ & &
\end{tabular}

$\mathrm{FC}=$ Frecuencia cardíaca $; \mathrm{EEM}=$ Eje eléctrico medio $; \mathrm{Pa}=$ amplitud de onda $\mathrm{P} ; \mathrm{Pd}=$ Duración de onda P; PR = intervalo P-R; QRSa =amplitud del complejo QRS; QRSd = Duración del complejo QRS; QT = Intervalo Q-T 
Tabla 3. Segmento S-T (ST; mv) expresado en media $\pm E E$ de las mismas perras de la Tabla 1.

\begin{tabular}{|c|c|c|c|c|c|c|}
\hline \multirow{3}{*}{ Parámetro } & \multicolumn{6}{|c|}{ Días de tratamiento } \\
\cline { 2 - 7 } & \multicolumn{2}{|c|}{ Día 0 } & \multicolumn{2}{c|}{ Día 3 } & \multicolumn{2}{c|}{ Día 6 } \\
\cline { 2 - 7 } & GM & CO & GM & CO & GM & CO \\
\hline ST & $-0,02 \pm 0,01$ & $-0,03 \pm 0,02$ & $-0,02 \pm 0,01$ & $-0,01 \pm 0,02$ & $-0,04 \pm 0,01$ & $-0,03 \pm 0,02$ \\
\hline
\end{tabular}

\section{Discusión}

En el presente estudio, la PAS progresivamente disminuyó durante la gestación normal, como ha sido reportado anteriormente en las hembras caninas (10); sin embargo, este mismo parámetro creció durante la inducción farmacológica del aborto. Este hallazgo podría estar en línea con reportes previos en medicina humana, en los cuales el incremento de la PAS materna se asocia con una variedad de complicaciones obstétricas $(11,12)$.

Adicionalmente, el flujo sanguíneo uterino disminuyó en el GM con respecto al CO, fenómeno reflejado en la falta de incremento de las VMAX, VMIN y GP de la arteria uterina y la falta de descenso del IR, que por el contrario, se incrementó en las hembras tratadas. El crecimiento de las velocidades del flujo sanguíneo uterino en la gestación normal, sumada a la caída del IR, coinciden con lo descripto en caninos (2,3). Esto puede ser explicado por el incremento en la densidad de capilares y vasos sanguíneos a lo largo de la preñez (33). La similitud entre los valores de las arterias uterinas derecha e izquierda probablemente se deba a la distribución pareja de los fetos en ambos cuernos uterinos (23).

Algo similar sucede con el IR de la arteria umbilical y el IR de la aorta abdominal fetal. Ambos índices decrecen durante la preñez canina normal (2,3), como sucedió en este experimento, indicando una eficiente irrigación fetal. Sin embargo, las hembras que abortaron 
tuvieron valores más altos en estos índices, posiblemente resultado de una inadecuada perfusión de los fetos (34).

El EFS presentó valores menores en el CO, probablemente debido a que el EFS se calcula en base a la PAS, que fue menor en este mismo grupo (19). El acortamiento presentado por el PE en el GM podría deberse a una disminución en la precarga (35).

Hasta donde conocemos, esta es la primera vez que se describen parámetros cardiovasculares durante un modelo experimental de aborto inducido en caninos. Los resultados en estas perras fueron similares a los ya observados en humanos (36). Este estudio preliminar parece confirmar que en la especie canina, los parámetros analizados pueden predecir un desenlace gestacional adverso. Si esto se confirma, los casos clínicos de gestación anormal comúnmente observados podrían diagnosticarse de manera temprana y eventualmente prevenirse antes de que aparezcan otros signos de compromiso gestacional. A pesar de que este modelo farmacológico fue útil para desarrollar y evaluar los cambios durante la interrupción de la preñez, se necesitarían más estudios con casos de gestación anormal espontánea para confirmar estos resultados experimentales.

En conclusión, en este modelo experimental de gestación anormal canina, algunos parámetros cardiovasculares difieren de la normalidad antes de que la preñez se interrumpa.

\section{Bibliografía}

1. Kametas NA, McAuliffe F, Hancock J, Chambers J, Nicolaides KH. Maternal left ventricular mass and diastolic function during pregnancy. Ultrasound Obstet Gynecol 2001;18:460-6. 
2. Nautrup CP. Doppler ultrasonography of canine maternal and fetal arteries during normal gestation. Journal of Reproduction and Fertility 1998;112:301-314.

3. Di Salvo P, Bocci F, Polisca A. Doppler evaluation of maternaland fetal vessels during normal gestation in the bitch.Res Vet Sci 2006;81:382-8.

4. Blanco PG, Arias DO, Gobello C. Doppler ultrasound in canine pregnancy. J Ultrasound Med 2008;27:1745-50.

5. Dickey RP. Doppler ultrasound investigation of uterine and ovarian blood flow in infertility and early pregnancy. Hum Reprod Update 1997;3:467-503.

6. Goswamy RK, Steptoe PC. Doppler ultrasound studiesof the uterine artery in spontaneous ovarian cycle. Hum Reprod 1988;3:721-6.

7. Papageorghiou AT, Yu CKH, Nicolaides KH. The role of uterine artery Doppler in predicting adverse pregnancy outcome. Best Pract Res Clin Obstet Gynaecol 2004;18;383-96.

8. Czajkowski K, Sienko J, Mogilinski M, Bros M, Szczecina R,Czajkowska A. Uteroplacental circulation in earlypregnancy complicated by threatened abortion supplemented with vaginal micronized progesterone or oral dydrogesterone. Fertil Steril 2007;87:613-18.

9. Miranda SA, Domingues SF. Conceptus ecobiometry and triplex Doppler ultrasonography of uterine and umbilical arteries for assessment of fetal viability in dogs. Theriogenology 2010;74:608-17.

10. Blanco PG, Tórtora M, Rodríguez R, Arias D, Gobello C. Ultrasonographic assessment of maternal cardiac function and peripheral circulation during normal gestation in dogs. Vet $\mathbf{J}$ 2010; doi:10.1016/j.tvj1.2010.08.013. 
11. Easterling TR, Watts DH, Schmucker BC, Benedetti TJ. Measurement of cardiac output during pregnancy: validation of Doppler technique and clinical observations in preeclampsia. ObstetGynecol 1987;69:845-50.

12. Vasapollo B, Novelli GP, Valensise H. Total vascular resistance and left ventricular morphology as screening tools for complications in pregnancy. Hypertension 2008;51: 10206.

13. Khaw A, Kametas NA, Turan OM, Bamfo JE, Nicolaides KH. Maternal cardiac function and uterine artery Doppler at 11-14 weeks in the prediction of pre-eclampsia in nulliparous women. BJOG - Int J Obstet Gyn 2008;115:369-76.

14. Corrada Y, Rodriguez R, Tortora M, Arias D, Gobello C. A combination of oral cabergoline and double cloprostenol injections to produce third-quarter gestation termination in the bitch. J Am Anim Hosp Assoc 2006;42:366-70.

15. Chetboul V, Tidholm A, Nicolle A, Sampedrano CC, Gouni V, Pouchelon JL, Lefebvre HP, Concordet D. Effects of animal position and number of repeated measurements on selected two-dimensional and M-mode echocardiographic variables in healthy dogs. J Am Vet Med A 2005;227:743-7.

16. Thomas WP, Gaber CE, Jacobs GJ, Kaplan PM, Lombard CW, Moise NS, Moses BL. Recommendations for standards in transthoracic two-dimensional echocardiography in the dog and cat. Echocardiography Committee of the Specialty of Cardiology, American College of Veterinary Internal Medicine. J Vet Intern Med 1993;7:247-52.

17. Kienle RD. Echocardiography. En: Kittelson MD, Kienle RD, editores. Small animal cardiovascular medicine textbook. St. Louis, Mosby; 1998. p. 95-117.

18. Boon J. The echocardiographyc examination. En: Boon JA, editora. Manual of Veterinary Echocardiography. Media, Williams and Wilkins; 1998. p. 35-128. 
19. Vuille C, Weyman AE. Left ventricle I: general considerations, assessment of chamber size and function.En: Weyman AE, editor. Principles and practice of echocardiography. Media, Williams and Wilkins;1994. p. 575-624.

20. Estrada A, Maisenbacher H. Calculation of stenotic valve area. J Vet Cardiol 2006;8:4953.

21. Gottdiener JS, Bednarz J, Devereux R, Gardin J, Klein A, Manning WJ, Morehead A, Kitzman D, Oh J, Quinones M, Schiller NB, Stein JH, Weissman NJ. American Society of Echocardiography recommendations for use of echocardiography in clinical trials. J Am Soc Echocardiog 2004;17:1086-119.

22. Littman MP, Fox PR. Systemic hypertension: recognition and treatment. En: Fox P, Sisson D, Moïse NS, editores. Textbook of canine and feline cardiology. Philadelphia, WB Saunders Co;1999. p. 795-813.

23. England GCW. Ultrasonograpic assessment of abnormal pregnancy. Ultrasonography 1998;28:849-67.

24. England GC, Russo M. Ultrasonographic characteristicsof early pregnancy failure in bitches. Theriogenology 2006;66:1694-8.

25. Alvarez-Clau A, Liste F. Ultrasonographic characterizationof the uterine artery in the nonestrus bitch. Ultrasound Med Biol 2005;3:1583-7.

26. Fong KW, Ohlsson A, Hannah ME, Grisaru S, Kingdom J, Cohen H, Ryan M, Windrim R, Foster G, Amankwah K. Prediction of perinatal outcome in fetuses suspected to have intrauterine growth restriction: Doppler US study of fetal cerebral, renal, and umbilical arteries. Radiology 1999;213:681-9.

27. Morales-Roselló J. Doppler sonography of normal fetal vertebral and internal carotid arteries during pregnancy. J Clin Ultrasound 2002;30:257-63. 
28. Beccaglia M, Faustini M, Luvoni GC. Ultrasonographic study of deep portion of diencephalo-telencephalic vesicle for the determination of gestational age of the canine foetus. Reprod Domest Anim 2008;43:367-70.

29. Verstegen JP, Silva LD, Onclin K, Donnay I. Echocardiographic study of heart rate in dog and cat fetuses in utero. J Reprod Fertil Suppl 1993;47:175-80.

30. Ferasin L, Ferasin H, Little CJ. Lack of correlation between canine heart rate and body size in veterinary clinical practice. J Small Anim Pract 2010;51:412-8.

31. Kittleson MD. Electrocardiography. En: Kittelson MD, Kienle RD, editores. Small animal cardiovascular medicine textbook. St. Louis, Mosby;1998.p. 72-94.

32. Tilley LP. The approach to the electrocardiogram. En: Tilley LP, editor. Essentials of canine and feline electrocardiography. Media, Lippincott Williams \& Wilkins;1992. p. 40-50. 33. Miglino MA, Ambrosio CE, dos Santos Martins D, Valverde Wenceslau C, Pfarrer C, Leiser R. The carnivore pregnancy: the development of the embryo and fetal membranes. Theriogenology 2006;66:1699-702.

34. Ghosh GS, Gudmundsson S. Uterine and umbilical artery Doppler are comparable in predicting perinatal outcome of growth-restricted fetuses. BJOG - Int J Obstet Gy 2009;116:424-30.

35. Atkins CE, Snyder PS. Systolic time intervals and their derivatives for evaluation of cardiac function. J Vet Intern Med 1992;6:55-63.

36. Strigini FA, Lencioni G, De Luca G, Lombardo M, Bianchi F, Genazzani AR.Uterine artery velocimetry and spontaneous preterm delivery. Obstet Gynecol 1995;85:374-7. 


\section{CAPÍTULO III}

\section{EVALUACIÓN ULTRASONOGRÁFICA DOPPLER DEL FLUJO SANGUÍNEO MATERNO-FETAL EN LA GESTACIÓN CANINA ANORMAL}

\section{Introducción}

La ultrasonografía Doppler se ha usado para evaluar el flujo sanguíneo materno y fetal durante la gestación en muchas especies (1). Esta técnica no invasiva evalúa la velocidad y el índice de resistencia (IR) del flujo de los vasos sanguíneos, datos que reflejan la resistencia vascular del área distal a la medición. Los IR de las arterias uteroplacentarias, umbilical, aorta y carótida común fetales, decrecen progresivamente a través de la gestación normal (2-5), garantizando una perfusión adecuada de la placenta y los órganos fetales.

En mujeres embarazadas, el Doppler se ha convertido en una técnica de uso rutinario para identificar el compromiso fetal asociado con una circulación uteroplacentaria y fetal deficientes. Un incremento en el IR de la arteria uterina y umbilical indica un alto riesgo de muerte fetal y perinatal (6-8). En mujeres embarazadas e hipertensas, el aumento de la resistencia vascular placentaria se asocia con hipoxia fetal y retardo del crecimiento intrauterino $(9,10)$. Más aún, los embarazos con ondas de morfología anormal en la arteria umbilical dan a luz más prematuramente que aquellas con ondas normales. Los bebés con índices Doppler anormales en esta misma arteria también tienen una mayor frecuencia de complicaciones neonatales (11).

En humanos, el examen Doppler del flujo sanguíneo fetal también provee información sobre las respuestas reflejas del sistema cardiovascular fetal. En este sentido, la evaluación de la 
aorta abdominal fetal aporta información sobre la circulación periférica de los miembros inferiores, del bazo, los músculos esqueléticos y el mesenterio (12). Más aún, el oligohidramnios en fetos con hipoxia prolongada es consecuencia de una pobre irrigación renal, que puede evidenciarse con la evaluación Doppler de la arteria renal fetal (13). Adicionalmente, la resistencia de la carótida interna fetal permite predecir la aparición de una FC fetal anormal (14). Algunos reportes han sugerido que una velocidad anormal en la arteria umbilical o en los vasos fetales puede incluso preceder a la desaceleración de la FC fetal (15). Asimismo, otros reportes sostienen que esta última variable no parece tener ninguna ventaja sobre la velocimetría Doppler (16).

Tradicionalmente, la evaluación ultrasonográfica de la biometría fetal y la FC fetal antes del parto han sido utilizadas para evaluar el estado de los fetos caninos. El tamaño del feto, su tasa de crecimiento, sus movimientos y la caída de su FC son los principales parámetros usados (17-19). Las medidas Doppler del flujo sanguíneo uterino, umbilical y fetal en las gestaciones patológicas no han sido reportadas aún en perros. Un modelo experimental de gestación anormal farmacológicamente inducida sugirió que el IR de la arteria uterina podría predecir el compromiso gestacional y el aborto inminente en esta especie (20). Sin embargo, no hay información disponible en casos de gestación patológica canina espontánea. Por esta razón, el objetivo de este trabajo fue reportar los cambios en el flujo sanguíneo de la arteria uterina, arteria umbilical, aorta abdominal fetal, renal fetal y carótida interna fetal en la gestación canina anormal. 


\section{Materiales y métodos}

Animales y seguimiento

Para este experimento se reclutaron veintidós perras preñadas puras (primíparas o multíparas), clínicamente sanas, de 1-8 años de edad, de 3-40 kg de peso. Todos los animales fueron negativos a Brucella canis y clasificados retrospectivamente en normales o anormales. El grupo anormal $(\mathrm{GA} ; \mathrm{n}=11)$ incluyó animales cuya gestación se interrumpió espontáneamente entre el día 52 y 60 de preñez (desde el pico estimado de LH) o que presentaron una mortalidad perinatal mayor al $60 \%$ de la camada $(21,22)$. En el grupo control $(G N ; n=11)$ las perras parieron cachorros sanos a término. Las hembras fueron evaluadas ultrasonográficamente cada 10 días del día 20 al 50 de preñez, definiendo el día 0 (pico de LH) como el primer día de citología vaginal típica de estro (23). La citología se realizó y evaluó de la misma manera que en el Capítulo 1. Ninguna de las perras incluidas presentó distocia o dio a luz por cesárea. El tamaño de camada varió de 2 a 11 cachorros. Este estudio fue aprobado por el Comité de Cuidado y Uso Animal de esta Institución.

\section{Evaluaciones ultrasonográficas}

Por medio de ultrasonografía bidimensional (Toshiba Core Vision Pro, Japón) se diagnosticó la preñez, tamaño de camada, muerte fetal e interrupción de la gestación, usando un transductor lineal de 5-8 MHz (24,25). Los exámenes con Doppler color y pulsado de las arterias uterinas se llevaron a cabo colocando las hembras en decúbito lateral, con el pelo del vientre rasurado. Se colocó gel sobre la piel para mejorar el acoplamiento acústico. El registro 
Doppler se efectuó según lo previamente publicado por Álvarez Clau y Liste (2005; 26). Para minimizar la variabilidad, un único operador registró tres ondas consecutivas y uniformes. Se midieron la velocidad de pico sistólico (VMAX) y la velocidad de flujo de fin de diástole (VMIN). El IR [(VMAX - VMIN)/VMAX] y el gradiente de presión (GP) se calcularon automáticamente (27).

En ambos grupos se realizó una ecocardiografía en modo M y Doppler en el feto más caudal del cuerno uterino derecho. Las arterias umbilical, aorta abdominal, renal y carótida interna fetales se evaluaron con Doppler color y pulsado (3). Para efectuar el Doppler de la arteria fetal renal, se obtuvo un corte longitudinal del riñón fetal. Luego, se detectó la arteria renal en el hilio del órgano (28). La carótida interna fetal se detectó a cada lado de las vesículas diencéfalo-telencefálicas, según lo publicado en trabajos anteriores $(29,30)$. Se registraron tres ondas uniformes y consecutivas de cada arteria para medir VMAX y VMIN. El IR y GP se calcularon automáticamente. La FC fetal ser registró con ecocardiografía en modo M y se promediaron tres mediciones (31). El último examen en todas las pacientes fue $4 \pm 0,9$ días (media $\pm \mathrm{EE})$ antes del aborto o parto.

\section{Análisis estadístico}

Con el fin de constatar la comparabilidad de ambos grupos en función de la edad, peso, parición y tamaño de camada se efectuó un test de Student. Los valores de VMAX, VMIN, IR y GP de las arterias uterina, umbilical, aorta abdominal fetal, arteria renal fetal y carótida interna fetal se transformaron a porcentaje de cambio [(Valor final - valor inicial / valor inicial) x 100]. Estos valores junto con la FC fetal se compararon entre los grupos (normal vs. 
anormal) por medio de un ANOVA de medidas repetidas seguido de un test de Tukey (SPSS 17.0, SPSS Inc. Chicago, IL, USA).

\section{Resultados}

Las evaluaciones ultrasonográficas se realizaron con éxito en todas las hembras durante períodos no mayores a 30 minutos. Ambos grupos de pacientes resultaron similares en cuanto a su peso, edad, parición y tamaño de camada $(\mathrm{p}>0,1)$. No se observaron diferencias entre los valores de las arterias uterinas derecha e izquierda $(\mathrm{p}>0,1)$, por lo cual los resultados se calcularon con las mediciones de uno de los dos vasos.

Los porcentajes de cambio de las VMAX y GP de la arteria uterina se incrementaron $(\mathrm{p}<$ 0,01) a lo largo del estudio independientemente del grupo (Tabla 1). Por el contrario, en esta misma arteria, se encontró una interacción entre tiempo y grupo en los porcentajes de cambio de la VMIN $(\mathrm{p}<0,01)$ y el IR $(\mathrm{p}<0,01)$. Aunque en ambos grupos la VMIN aumentó hasta el día 40, este parámetro permaneció constante en los animales del GA a partir de ese momento (Figura 1). En este último grupo, el IR de la arteria uterina creció desde el día 40 en adelante (Figura 2).

En el GA, sólo 7 perras alcanzaron el día 60 preñez y fueron evaluadas por medio de ecocardiografía fetal en modo M y Doppler. El IR de la arteria umbilical se modificó a lo largo del tiempo dependiendo del grupo $(\mathrm{p}<0,05)$ difiriendo entre ellos desde el día 50 en adelante (Figura 3). El porcentaje de cambio de la VMIN de esta arteria difirió entre los animales normales y anormales $(\mathrm{p}=0,01)$, mientras que aquellos de VMAX y GP no difirieron ( $p$ > 0,05; Tabla 2). Los porcentajes de cambio de la VMAX, VMIN y GP de la arteria umbilical aumentaron durante el estudio $(\mathrm{p}<0,01)$ en todas las perras 
independientemente del grupo. Los porcentajes de cambio de la VMAX, VMIN y GP de la aorta abdominal fetal se incrementaron a lo largo del período de estudio $(\mathrm{p}<0,01)$ en ambos grupos, sin diferir entre ellos ( $p>0,05$; Tabla 3). Por el contrario, el porcentaje de cambio del IR de este vaso descendió durante el mismo período $(\mathrm{p}<0,01)$ en todas las perras.

En la arteria renal fetal se observó una interacción entre el tiempo y grupo para el porcentaje de cambio del IR ( $\mathrm{p}<0,05)$. En las perras normales, esta variable disminuyó a lo largo del estudio, mientras que en los animales anormales el IR creció desde el día 50 en adelante (Figura 4). En esta arteria, el porcentaje de cambio del GP difirió entre el GN y el GA (p < 0,05), mientras que los de la VMAX y VMIN no lo hicieron ( $p>0,05)$. Contrariamente, el porcentaje de cambio de la VMIN creció progresivamente durante el experimento en ambos grupos $(\mathrm{p}<0,01$; Tabla 4).

Tampoco se encontraron diferencias entre grupos ( $p>0,05$; Tabla 5) para el porcentaje de cambio de la VMAX, VMIN, GP e IR de la carótida interna fetal. Los tres primeros parámetros se incrementaron $(\mathrm{p}<0,01)$ en tanto que el cuarto disminuyó en el curso del estudio en todas las pacientes $(\mathrm{p}<0,01)$. Finalmente, la FC fetal en el GA fue más alta que en el GN (p < 0,05; Tabla 6). 


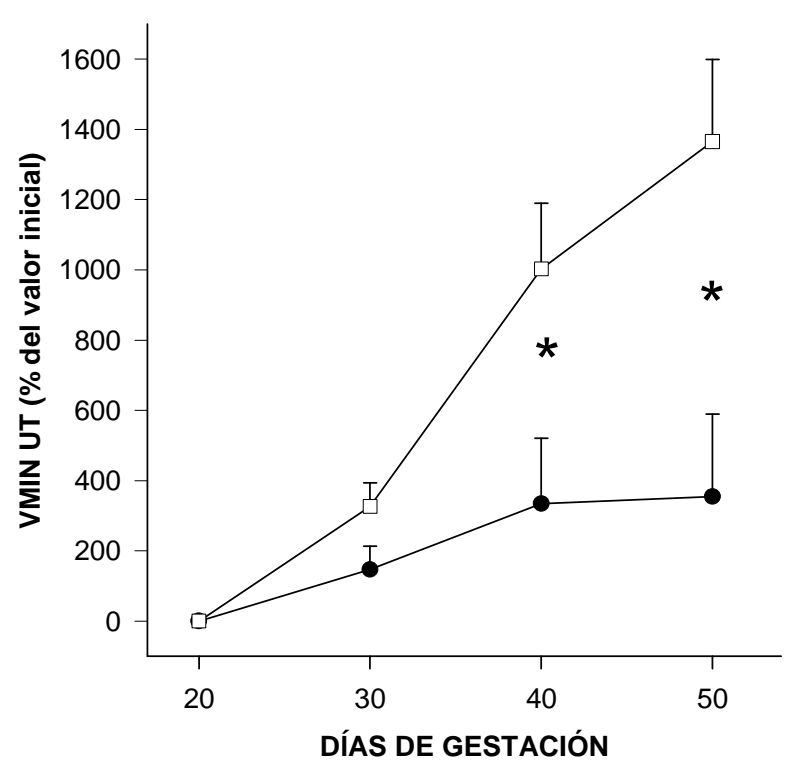

Figura 1. Porcentaje de cambio de la velocidad de flujo de fin de diástole de la arteria uterina (VMIN UT; media \pm EE) de 11 perras preñadas anormales (GA) y 11 perras preñadas normales (GN) evaluadas cada 10 días desde el día 20 al 50 del pico estimado de LH.

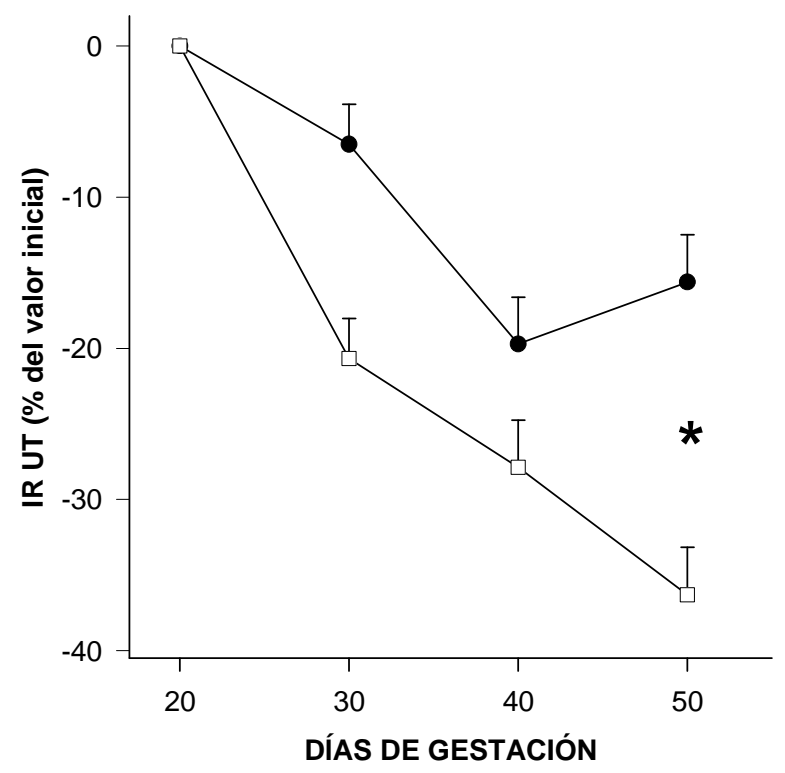

Figura 2. Porcentaje de cambio del índice de resistencia de la arteria uterina (IR UT; media $\pm E E$ ) de las mismas perras de la Figura 1. 


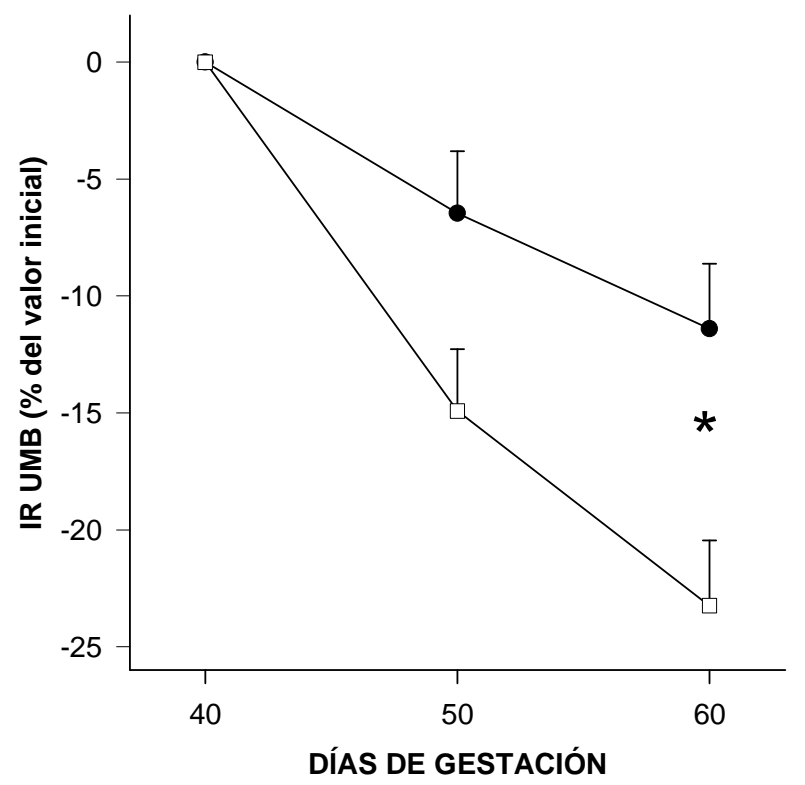

Figura 3. Porcentaje de cambio del índice de resistencia de la arteria umbilical (IR UMB; media $\pm \mathrm{EE})$ de 7 perras preñadas anormales $(\mathrm{GA})$ y 11 perras preñadas normales $(\mathrm{GN})$ de la

\section{Figura 1.}

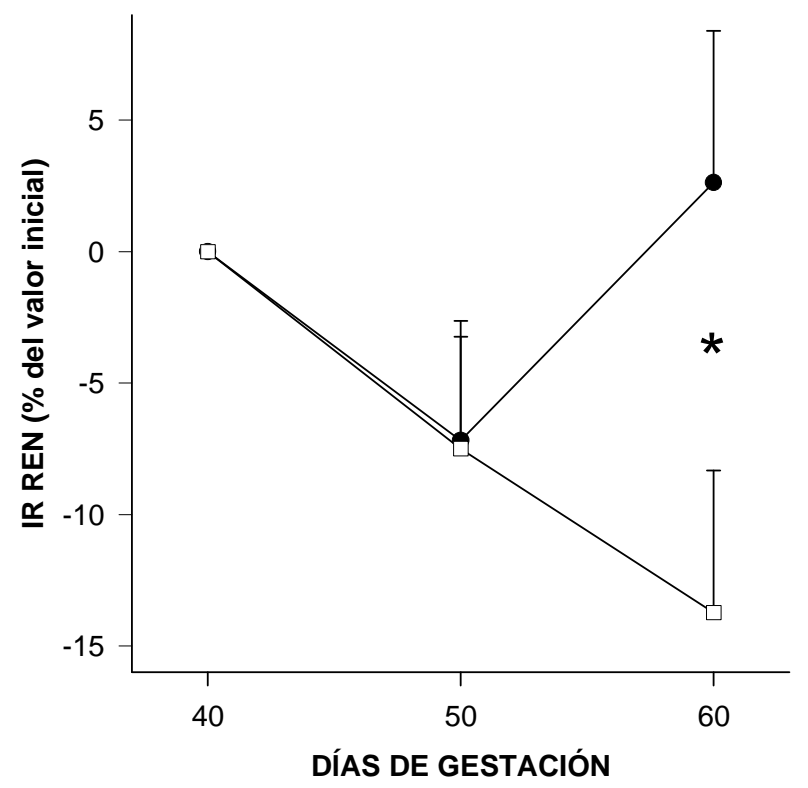

Figura 4. Porcentaje de cambio del índice de resistencia de la arteria renal fetal (IR REN; media $\pm E E)$ de 7 perras preñadas anormales $(\mathrm{GA})$ y 11 perras preñadas normales $(\mathrm{GN})$ de la Figura 1. 
Tabla 1. Porcentaje de cambio de la velocidad de pico sistólico (VMAX) y gradiente de presión (GP) de la arteria uterina (media $\pm \mathrm{EE}$ ) de 11 perras preñadas anormales $(\mathrm{GA})$ y 11 perras preñadas normales (GN) evaluadas cada 10 días desde el día 20 al 50 del pico estimado de LH.

\begin{tabular}{|c|c|c|c|c|}
\hline \multirow{2}{*}{ Días de gestación } & \multicolumn{2}{|c|}{ VMAX } & \multicolumn{2}{c|}{ GP } \\
\cline { 2 - 5 } & GA & GN & GA & GN \\
\hline 30 & $67 \pm 24$ & $66 \pm 24$ & $237 \pm 79$ & $228 \pm 79$ \\
\hline 40 & $103 \pm 40$ & $205 \pm 40$ & $424 \pm 244$ & $1004 \pm 244$ \\
\hline 50 & $120 \pm 48$ & $242 \pm 48$ & $643 \pm 408$ & $1306 \pm 408$ \\
\hline
\end{tabular}

Tabla 2. Porcentaje de cambio de la velocidad de pico sistólico (VMAX), velocidad de flujo de fin de diástole (VMIN) y gradiente de presión (GP) la arteria umbilical (media $\pm E E$ ) de 7 perras preñadas anormales (GA) y 11 perras preñadas normales (GN) de la Tabla 1.

\begin{tabular}{|c|c|c|c|c|c|c|}
\hline \multirow{2}{*}{$\begin{array}{c}\text { Días de } \\
\text { gestación }\end{array}$} & \multicolumn{2}{|c|}{ VMAX } & \multicolumn{2}{c|}{ VMIN } & \multicolumn{2}{c|}{ GP } \\
\cline { 2 - 7 } & GA & GN & GA & GN & GA & GN \\
\hline 50 & $256 \pm 137$ & $523 \pm 109$ & $244 \pm 136$ & $618 \pm 109$ & $244 \pm 174$ & $800 \pm 146$ \\
\hline 60 & $483 \pm 231$ & $770 \pm 184$ & $765 \pm 169$ & $1155 \pm 135$ & $983 \pm 236$ & $1060 \pm 197$ \\
\hline
\end{tabular}

Tabla 3. Porcentaje de cambio de la velocidad de pico sistólico (VMAX), velocidad de flujo de fin de diástole (VMIN), índice de resistencia (IR) y gradiente de presión (GP) de la aorta abdominal fetal (media \pm EE) de las mismas perras de la Tabla 2.

\begin{tabular}{|c|c|c|c|c|c|c|c|c|}
\hline \multirow{2}{*}{$\begin{array}{c}\text { Días de } \\
\text { gestación }\end{array}$} & \multicolumn{2}{|c|}{ VMAX } & \multicolumn{2}{c|}{ VMIN } & \multicolumn{2}{c|}{ IR } & \multicolumn{2}{c|}{ GP } \\
\cline { 2 - 8 } & GA & GN & GA & GN & GA & GN & GA & GN \\
\hline 50 & $80 \pm 54$ & $168 \pm 51$ & $95 \pm 56$ & $375 \pm 52$ & $-3 \pm 2$ & $-9 \pm 2$ & $212 \pm 120$ & $542 \pm 112$ \\
\hline 60 & $135 \pm 58$ & $199 \pm 54$ & $593 \pm 138$ & $750 \pm 129$ & $-13 \pm 3$ & $-14 \pm 2$ & $336 \pm 165$ & $696 \pm 155$ \\
\hline
\end{tabular}


Tabla 4. Porcentaje de cambio de la velocidad de pico sistólico (VMAX), velocidad de flujo de fin de diástole (VMIN), índice de resistencia (IR) y gradiente de presión (GP) de la arteria renal fetal (media $\pm E E)$ de las mismas perras de la Tabla 2.

\begin{tabular}{|c|c|c|c|c|c|c|}
\hline \multirow{2}{*}{ Días de gestación } & \multicolumn{2}{|c|}{ VMAX } & \multicolumn{2}{c|}{ VMIN } & \multicolumn{2}{c|}{ GP } \\
\cline { 2 - 7 } & GA & GN & GA & GN & GA & GN \\
\hline 50 & $64 \pm 23$ & $64 \pm 23$ & $87 \pm 39$ & $121 \pm 36$ & $8.3 \pm 17$ & $54 \pm 15$ \\
\hline 60 & $252 \pm 117$ & $113 \pm 117$ & $125 \pm 39$ & $192 \pm 36$ & $10 \pm 37$ & $100 \pm 34$ \\
\hline
\end{tabular}

Tabla 5. Porcentaje de cambio de la velocidad de pico sistólico (VMAX), velocidad de flujo de fin de diástole (VMIN), índice de resistencia (IR) y gradiente de presión (GP) de la carótida interna fetal (media \pm EE) de las mismas perras de la Tabla 2.

\begin{tabular}{|c|c|c|c|c|c|c|c|c|}
\hline \multirow{2}{*}{$\begin{array}{c}\text { Días de } \\
\text { gestación }\end{array}$} & \multicolumn{2}{|c|}{ VMAX } & \multicolumn{2}{c|}{ VMIN } & \multicolumn{2}{c|}{ IR } & \multicolumn{2}{c|}{ GP } \\
\cline { 2 - 9 } & GA & GN & GA & GN & GA & GN & GA & GN \\
\hline 50 & $36 \pm 25$ & $58 \pm 24$ & $75 \pm 40$ & $85 \pm 40$ & $-9 \pm 6$ & $-9 \pm 6$ & $14 \pm 21$ & $22 \pm 18$ \\
\hline 60 & $67 \pm 23$ & $78 \pm 22$ & $107 \pm 46$ & $185 \pm 46$ & $-16 \pm 4$ & $-20 \pm 4$ & $57 \pm 28$ & $55 \pm 25$ \\
\hline
\end{tabular}

Tabla 6. Frecuencia cardíaca fetal (lpm; media $\pm E E)$ de las mismas perras de la Tabla 2.

\begin{tabular}{|c|c|c|}
\hline Días de gestación & GA & GN \\
\hline 40 & $242 \pm 2$ & $233 \pm 2$ \\
\hline 50 & $239 \pm 3$ & $231 \pm 3$ \\
\hline 60 & $223 \pm 6$ & $217 \pm 6$ \\
\hline
\end{tabular}

\section{Discusión}

Como se esperaba en este estudio, el IR de la arteria uterina se incrementó en las preñeces caninas patológicas y disminuyó en las normales. Estas diferencias no podrían atribuirse al peso, edad o tamaño de camada, en tanto que demostraron no influir en los parámetros 
Doppler (32). En todos los animales, la VMAX, VMIN y GP de esta arteria crecieron progresivamente durante la gestación, como se ha descripto anteriormente para las arterias uteroplacentarias $(2,3)$. Resulta interesante que en las perras anormales, la VMIN cesó de crecer cuando el IR cambió su tendencia normal descendente a una ascendente. Estos hallazgos están en línea con los de humanos, donde el componente diastólico del flujo sanguíneo reducido y el IR aumentado reflejan una alta resistencia a nivel de la microcirculación placentaria $(6,9,33)$. En este sentido, la porción diastólica del ciclo cardíaco parece ser más precisa que la porción sistólica a la hora de reflejar los cambios en el IR. Estos resultados también están en línea con un modelo experimental de gestación canina anormal, donde el IR creció progresivamente hasta el aborto (20). No obstante, hasta donde se conoce, este es el primer reporte del flujo sanguíneo uterino durante la gestación anormal espontánea en esta especie. Se postula que la invasión defectuosa del trofoblasto sería la causa subyacente de las ondas Doppler anormales de la arteria uterina, conduciendo a una insuficiencia vascular uteroplacentaria (34).

De acuerdo a reportes previos en animales normales, la VMAX, VMIN y GP de la arteria umbilical aumentan en el curso de la gestación, mientras que el IR decrece progresivamente $(2,3)$. Sin embargo, el IR fue más alto en las gestaciones anormales debido a un menor aumento de la VMIN. Estos hallazgos coinciden con reportes en medicina humana, donde esta arteria ha mostrado ser útil para identificar fetos que están en riesgo de vida $(9,11)$. También se ha sugerido que un flujo diastólico ausente o incluso reverso en la arteria umbilical se asocia con una alta tasa de mortalidad perinatal y alta morbilidad en los bebés sobrevivientes (34). Sin embargo, en el presente estudio, el flujo diastólico de esta arteria fue detectado por primera vez al día 40 de preñez y desde entonces nunca desapareció o se presentó negativo en ninguna de las perras. 
En ambos grupos de animales, el flujo sanguíneo de la aorta abdominal fetal se incrementó a lo largo de la gestación, como ha sido ya reportado (2,3). Aunque no se encontraron diferencias significativas en el IR, esta variable fue levemente más alta en el grupo anormal, lo cual puede indicar una perfusión deficiente en el bazo, intestinos y miembros posteriores (12).

Hasta donde sabemos, este es el primer reporte del flujo sanguíneo de las arterias renal y carótida interna fetales en la gestación canina normal y anormal. Ambas arterias fueron detectadas por primera vez al día 40 de preñez en ambos grupos, y en ese momento mostraron sólo el componente sistólico. Su flujo aumentó durante el estudio en el grupo normal, lo cual coincide con reportes previos en mujeres $(13,29)$. Por el contrario, en el GA, el incremento del IR de la arteria renal fetal indicaría una reducción en el porcentaje del gasto cardíaco fetal dirigido hacia los riñones y la consecuente perfusión renal pobre. Este hallazgo también coincide con resultados en obstetricia humana, donde el aumento del IR se relaciona con una menor producción de orina y líquido amniótico (35). La carótida interna mostró un alza en el flujo sanguíneo en el grupo patológico. En casos de insuficiencia placentaria, el feto dirige su flujo sanguíneo hacia órganos vitales como el cerebro. El aumento de la perfusión cerebral se conoce como "brain-sparing effect" (36), y podría ser el resultado de una redistribución del gasto cardíaco durante la hipoxia fetal (15). Este fenómeno podría explicar que el IR de la carótida interna no haya cambiado en el curso de la preñez patológica.

Los movimientos fetales y la FC fetal generalmente decrecen como resultado del estrés y la hipoxia en la gestación canina (37). Sabiendo que la hipoxia fetal inicialmente afecta órganos no vitales (38), el corazón como órgano vital resulta afectado (por ejemplo, con desaceleraciones) después del útero, riñón y flujo sanguíneo umbilical. Trabajos en medicina humana han sugerido que la vasodilatación de las arterias fetales cerebrales tanto como los 
cambios en los vasos umbilicales y en otros, ocurren semanas antes del comienzo de la bradicardia fetal $(15,28)$. Es interesante destacar que en la mayoría de las perras anormales, la FC fetal no se desaceleró y fue incluso más alta que en las hembras normales. Una explicación para esta aceleración sería que la maduración del control cardíaco vagal depende del peso fetal, y un bajo peso resulta de una irrigación pobre del feto. Más aún, la concentración de catecolaminas plasmáticas asciende progresivamente con grados mayores de hipoxia (39).

En perros, la ecografía bidimensional ha sido ampliamente usada para evaluar el desarrollo y viabilidad de los fetos caninos, para estimar la edad gestacional y predecir la fecha de parto (18). Sin embargo, se han descripto pocos parámetros que sean indicativos de estrés fetal (40). Contando con el equipamiento adecuado, la evaluación del flujo sanguíneo materno y fetal canino es fácil de realizar y tiene un útil potencial informativo en gestaciones comprometidas. Estos resultados muestran que, en preñeces patológicas, las anormalidades en el trazado Doppler pueden aparecer antes que los cambios en la FC fetal. Esto pone énfasis en la importancia de los exámenes Doppler rutinarios durante la gestación canina. La evidencia del estrés fetal inminente puede contribuir a una adecuada toma de decisiones terapéuticas. En este aspecto, resultaría muy útil contar con los rangos de referencia normales de los parámetros vasculares en perras de razas pequeñas, medianas y grandes. Se necesitarían más trabajos incluyendo diferentes agentes etiológicos para describir en profundidad los cambios patológicos en el flujo sanguíneo materno - fetal durante la gestación anormal en esta especie. Concluimos que el flujo sanguíneo de las arterias uterina, umbilical y renal fetal difiere entre la gestación canina normal y anormal, permitiendo predecir un desenlace gestacional adverso. 


\section{Bibliografía}

1. Blanco PG, Arias DO, Gobello C. Doppler ultrasound in canine pregnancy. J Ultrasound Med 2008;27:1745-50.

2. Nautrup CP. Doppler ultrasonography of canine maternal and fetal arteries during normal gestation. Journal of Reproduction and Fertility 1998;112:301-314.

3. Di Salvo P, Bocci F, Polisca A. Doppler evaluation of maternaland fetal vessels during normal gestation in the bitch.Res Vet Sci 2006;81:382-8.

4. Miranda SA, Domingues SF. Conceptus ecobiometry and triplex Doppler ultrasonography of uterine and umbilical arteries for assessment of fetal viability in dogs. Theriogenology 2010;74:608-17.

5. Blanco PG, Tórtora M, Rodríguez R, Arias D, Gobello C. Ultrasonographic assessment of maternal cardiac function and peripheral circulation during normal gestation in dogs. Vet $\mathrm{J}$ 2010; doi:10.1016/j.tvj1.2010.08.013.

6. Dickey RP. Doppler ultrasound investigation of uterine and ovarian blood flow in infertility and early pregnancy. Hum Reprod Update 1997;3:467-503.

7. Papageorghiou AT, Yu CKH, Nicolaides KH. The role of uterine artery Doppler in predicting adverse pregnancy outcome. Best Pract Res Clin Obstet Gynaecol 2004;18;383-96.

8. Strigini FA, Lencioni G, De Luca G, Lombardo M, Bianchi F, Genazzani AR.Uterine artery velocimetry and spontaneous preterm delivery. Obstet Gynecol 1995;85:374-7.

9. Acharya G, Erkinaro T, Mäkikallio K, Lappalainen T, Rasanen J. Relationships among Doppler-derived umbilical artery absolute velocities, cardiac function, and placental volume blood flow and resistance in fetal sheep. Am J Physiol Heart Circ Physiol 2004;286:H126672. 
10. Ghosh GS, Gudmundsson S. Uterine and umbilical artery Doppler are comparable in predicting perinatal outcome of growth-restricted fetuses. BJOG - Int J Obstet Gy 2009;116:424-30.

11. Acharya G, Wilsgaard T, Berntsen GK, Maltau JM, Kiserud T. Reference ranges for serial measurements of umbilical artery Doppler indices in the second half of pregnancy. Am $\mathbf{J}$ Obstet Gynecol 2005;192:937-44.

12. Akalin-Sel T, Campbell S. Understanding the pathophysiology of intra-uterine growth retardation: the role of the 'lower limb reflex' in redistribution of blood flow. Eur J Obstet Gynecol Reprod Biol 2002;23:79-86.

13. Mari G, Kirshon B, Abuhamad A. Fetal renal artery flow velocity waveforms in normal pregnancies and pregnancies complicated by polyhydramnios and oligohydramnios. Obstet Gynecol 1993;81:560-4.

14. Groenenberg IA, Hop WC, Bogers JW, Santema JG, Wladimiroff JW. The predictive value of Doppler flow velocity waveforms in the development of abnormal fetal heart rate traces in intrauterine growth retardation: a longitudinal study. Early Hum Dev 1993;32:151-9. 15. Arduini D, Rizzo G, Romanini C. Changes of pulsatility index from fetal vessels preceding the onset of late decelerations in growth-retarded fetuses. Obstet Gynecol 1992;79:605-10.

16. Chan FY, Lam C, Lam YH, To WK, Pun TC, Lee CP. Umbilical artery Doppler velocimetry compared with fetal heart rate monitoring as a labor admission test. Eur J Obstet Gynecol Reprod Biol 1994;54:1-6.

17. Johnson CA. High-risk pregnancy and hypoluteoidism in the bitch. Theriogenology 2008a;70:1424-30. 
18. Kutzler MA, Yeager AE, Mohammed HO, Meyers-Wallen VN. Accuracy of canine parturition date prediction using fetal measurements obtained by ultrasonography. Theriogenology 2003;60:1309-17.

19. Zone MA, Wanke MM. Diagnosis of canine fetal health by ultrasonography. J Reprod Fertil Suppl 2001;57:215-9.

20. Blanco PG, Arias D, Rube A, Barrena JP, Corrada Y, Gobello C. An experimental model to study resistance index and systolic/diastolic ratio of uterine arteries in adverse canine pregnancy outcome. Reprod Domest Anim 2009;44:164-6.

21. Davidson AP. Approaches to Reducing Neonatal Mortality in Dogs. En: Concannon PW, England G, Verstegen J, Linde-Forsberg C, editores. Recent Advances in Small Animal Reproduction. Ithaca, New York, IVIS, 2003; www.ivis.org. Document number A1226.0303. 22. Münnich A. The pathological newborn in small animals: the neonate is not a small adult. Vet Res Commun 2008;32:S81-5.

23. Olson PN, Husted PW, Allen TA, Nett TM. Reproductive endocrinology and physiology of the bitch and queen. Vet Clin North Am Small Anim Pract 1984;14:927-46.

24. England GCW. Ultrasonograpic assessment of abnormalpregnancy. Ultrasonography 1998;28:849-67.

25. England GC, Russo M. Ultrasonographic characteristicsof early pregnancy failure in bitches. Theriogenology 2006;66:1694-8.

26. Alvarez-Clau A, Liste F. Ultrasonographic characterizationof the uterine artery in the nonestrus bitch. UltrasoundMed Biol 2005;3:1583-7.

27. Dickey RP. Doppler ultrasound investigation of uterine and ovarian blood flow in infertility and early pregnancy. Hum Reprod Update 1997;3:467-503. 
28. Fong KW, Ohlsson A, Hannah ME, Grisaru S, Kingdom J, Cohen H, Ryan M, Windrim R, Foster G, Amankwah K. Prediction of perinatal outcome in fetuses suspected to have intrauterine growth restriction: Doppler US study of fetal cerebral, renal, and umbilical arteries. Radiology 1999;213:681-9.

29. Morales-Roselló J. Doppler sonography of normal fetal vertebral and internal carotid arteries during pregnancy. J Clin Ultrasound 2002;30:257-63.

30. Beccaglia M, Faustini M, Luvoni GC. Ultrasonographic study of deep portion of diencephalo-telencephalic vesicle for the determination of gestational age of the canine foetus. Reprod Domest Anim 2008;43:367-70.

31. Verstegen-Onclin K, Verstegen J. Endocrinology of pregnancy in the dog: a review. Theriogenology 2008;70:291-9.

32. Geipel A, Hennemann F, Fimmers R, Willruth A, Lato K, Gembruch U, Berg C. Reference ranges for Doppler-assessment of uterine artery resistance and pulsatility indices in dichorionic twin pregnancies. Ultrasound Obstet Gynecol 2010;14. [Epub ahead of print]. 33. Cnossen JS, Morris RK, ter Riet G, Mol BW, van der Post JA, Coomarasamy A, Zwinderman AH, Robson SC, Bindels PJ, Kleijnen J, Khan KS. Use of uterine artery Doppler ultrasonography to predict pre-eclampsia and intrauterine growth restriction: a systematic review and bivariable meta-analysis. Can Med Assoc J 2008;178:701-11.

34. Viero S, Chaddha V, Alkazaleh F, Simchen MJ, Malik A, Kelly E, Windrim R, Kingdom JC. Prognostic value of placental ultrasound in pregnancies complicated by absent enddiastolic flow velocity in the umbilical arteries. Placenta 2004;25:735-41.

35. Stigter RH, Mulder EJ, Bruinse HW, Visser GH. Doppler studies on the fetal renal artery in the severely growth-restricted fetus. Ultrasound Obstet Gynecol 2001;18:141-5. 
36. Detti L, Akiyama M, Mari G. Doppler blood flow in obstetrics. Curr Opin Obstet Gynecol 2002;14:587-93.

37. Johnson CA. Pregnancy management in the bitch. Theriogenology 2008b;70:1412-7.

38. Monheit AG, Stone ML, Abitbol MM. Fetal heart rate and transcutaneous monitoring during experimentally induced hypoxia in the fetal dog. Pediatr Res 1998;23:548-52.

39. Gu W, Jones CT, Parer JT. Metabolic and cardiovascular effects on fetal sheep of sustained reduction of uterine blood flow. J Physiol 1985;368:109-29.

40. Traas AM. Surgical management of canine and feline dystocia. Theriogenology 2008;70:337-42. 


\section{CAPÍTULO IV}

\section{EVALUACIÓN ULTRASONOGRÁFICA DE LA MORFOLOGÍA Y FUNCIÓN CARDÍACA Y LA CIRCULACIÓN PERIFÉRICA MATERNAS DURANTE} LA GESTACIÓN CANINA ANORMAL

\section{Introducción}

La adaptación cardíaca materna durante la gestación juega un rol importante en la perfusión uterina destinada a garantizar el desarrollo fetal (1-3). Un descenso en la poscarga, un incremento de la función sistólica y una hipertrofia cardíaca son el resultado de las modificaciones hemodinámicas que ocurren durante la gestación canina normal (4,5). En esta especie, durante la segunda mitad de la gestación, la morfología cardíaca se caracteriza por un aumento en el diámetro diastólico del VI (DDVI), del tabique interventricular en diástole (TIVD) y sístole (TIVS), de la pared posterior en sístole (PPS) y una caída del diámetro sistólico del VI (DSVI) (4,5). Más aún, la fracción de acortamiento (FA) y el porcentaje medio de acortamiento circunferencial (\% MAC) aumentan desde el día 30 en adelante, mientras que el estrés de fin de sístole (EFS) disminuye durante el mismo período (5). Estos cambios funcionales estarían relacionados a una mejora en la función sistólica, un aumento en la volemia y una caída en la presión arterial sistémica (PAS) que ocurren durante la preñez. La reducción del índice de resistencia (IR) de la arteria uterina que tiene lugar en la gestación, representa una contribución del 20 al $26 \%$ en la caída de la resistencia vascular total $(1,5)$. Adicionalmente, la disminución en la resistencia periférica es el principal determinante del 
aumento en el volumen sistólico (VS) y del gasto cardíaco (GC), que caracteriza la preñez $(5,6)$.

En mujeres, la mala adaptación cardiovascular materna está altamente relacionada con un desenlace gestacional adverso (7-9). Existe evidencia de que la restricción del crecimiento intrauterino se asocia con una expansión defectuosa del espacio intravascular materno y una falta de aumento del GC (10).

En perros, la circulación periférica ha sido descripta a través del flujo sanguíneo uterino en un modelo experimental de gestación anormal (11). No obstante, no existen reportes sobre la estructura y función cardíaca durante la preñez anormal espontánea. La función y morfología cardíaca de las perras preñadas con altas probabilidades de tener complicaciones obstétricas podrían diferir de aquellas con un desenlace normal. Por esto, la evaluación de la estructura del VI materno con evidencia de una geometría alterada podría proveer una información pronóstica de vital importancia. En consecuencia, el objetivo del presente estudio fue describir los cambios en la morfología cardíaca, función sistólica y PAS durante la gestación anormal en perros.

\section{Materiales y métodos}

Animales y seguimiento

Para este estudio se incluyeron veinte perras preñadas de razas puras, de 1-6 años de edad, de 3-50 kg de peso, primíparas y multíparas. Todos los casos fueron negativos a Brucella canis y clasificados retrospectivamente como normales y anormales. El grupo anormal (GA; n = 10) 
incluyó animales cuya gestación se interrumpió espontáneamente entre el día 52 y 60 de preñez (desde el pico estimado de LH) o que presentaron una mortalidad perinatal mayor al $60 \%$ de la camada $(12,13)$. En el grupo control $(\mathrm{GN} ; \mathrm{n}=10)$ las perras parieron cachorros sanos a término. Las hembras fueron evaluadas ultrasonográficamente cada 10 días del día 10 al 50 de preñez, definiendo el día 0 (pico de LH) como el primer día de citología vaginal típica de estro (14). La citología se realizó y evaluó de la misma manera que en el Capítulo 1. Ninguna de las perras incluidas presentó distocia o dio a luz por cesárea. El tamaño de camada varió de 2 a 11 cachorros. Este estudio fue aprobado por el Comité de Cuidado y Uso Animal de esta Institución.

\section{Evaluaciones ecocardiográficas}

Se realizaron las evaluaciones ecocardiográficas bidimensionales, en modo M y Doppler con los animales en estación, utilizando un transductor $7 \mathrm{MHz}$ (Toshiba Core Vision Pro; 15). Sobre la piel rasurada, se aplicó gel para lograr un adecuado acoplamiento acústico. Las ventanas paraesternales utilizadas fueron las previamente descriptas por Thomas y col. $(1996 ; 16)$.

Se registraron las medidas de DDVI, DSVI, TIVD, TIVS, pared posterior en diástole (PPD), PPS y FA por medio del modo M (17). La FA se calculó como (DDVI-DSVI)/DDVI x 100 (18). La masa ventricular (MV), relación radio espesor (RRE), EFS y \% MAC se calcularon de acuerdo a las fórmulas correspondientes (17-19). Con el mismo modo ecocardiográfico, se registraron la relación Ao/AI (Ao/AI), período eyectivo (PE), amplitud aórtica (AA) y punto de separación septal E (PSSE; 17). 
Con ecocardiografía Doppler espectral se registró el VTI del flujo transaórtico a partir del cual se calcularon el VS y el GC $(18,20)$.

Para minimizar la variabilidad en las mediciones, todas fueron efectuadas por un único operador entrenado y siguiendo guías de recomendación específicas (16,21). Se promediaron tres valores de cada parámetro.

Presión sanguínea sistémica

La PAS se midió utilizando un equipo Doppler vascular (DV610 Medmega, Brasil). Se utilizaron manguitos cuyo ancho correspondió al $40 \%$ de la circunferencia total del miembro anterior (22). Los animales se colocaron en decúbito lateral y se promediaron tres registros.

Análisis estadístico

Para constatar la comparabilidad de ambos grupos en función de la edad, peso, parición y tamaño de camada, se efectuó un test de Student. Todas las variables fueron transformadas a porcentaje de cambio ([Valor final - Valor inicial / Valor inicial] x 100) y comparadas entre los grupos por medio de un ANOVA de medidas repetidas seguido de un test de Tukey (SPSS 17.0, SPSS Inc.).

\section{Resultados}

Ambos grupos de pacientes resultaron similares, considerando el peso, edad, parición y tamaño de camada $(\mathrm{p}>0,1)$. Se encontró una interacción entre el tiempo y el grupo en los 
porcentajes de cambio de la PPS (p < 0,05), DSVI $(\mathrm{p}<0,01)$, FA $(\mathrm{p}<0,05), \%$ MAC $(\mathrm{p}<$ 0,05) y EFS ( $\mathrm{p}<0,01)$ a lo largo del período de estudio. Al día 50 de gestación, el porcentaje de cambio de la PPS se incrementó en el grupo normal pero no en el anormal (Figura 1). Más aún, los porcentajes de cambio de la FA y del \% MAC también se incrementaron sólo en los animales normales desde el día 40 en adelante (Figuras 2 y 3). Contrariamente, el porcentaje de cambio del DSVI disminuyó desde el día 40 hasta el parto (Figura 4), mientras que el EFS cayó desde el día 40, únicamente en las preñeces normales (Figura 5). El TIVD, TIVS, DDVI, PPD, MV, RRE, Ao/AI, PE, AA, PSSE, VS, GC, FC y PAS no difirieron entre los grupos ( $p>0,05$; Tabla 1). Adicionalmente, la PAS mostró una tendencia descendente en el grupo normal pero no en el anormal ( $\mathrm{p}=0,08$; Figura 6).

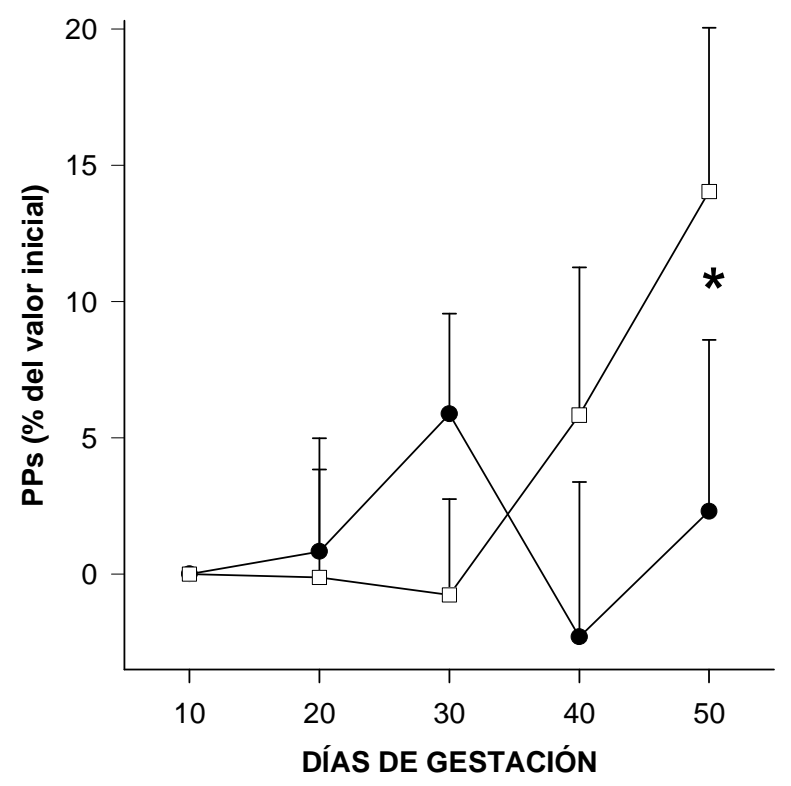

Figura 1: Porcentaje de cambio de la pared posterior en sístole (PPS; media $\pm E E)$ en perras preñadas anormales (círculos; $\mathrm{n}=10$ ) y normales (cuadrados; $\mathrm{n}=10$ ) evaluadas cada 10 días desde el día 10 al 50 de gestación. 


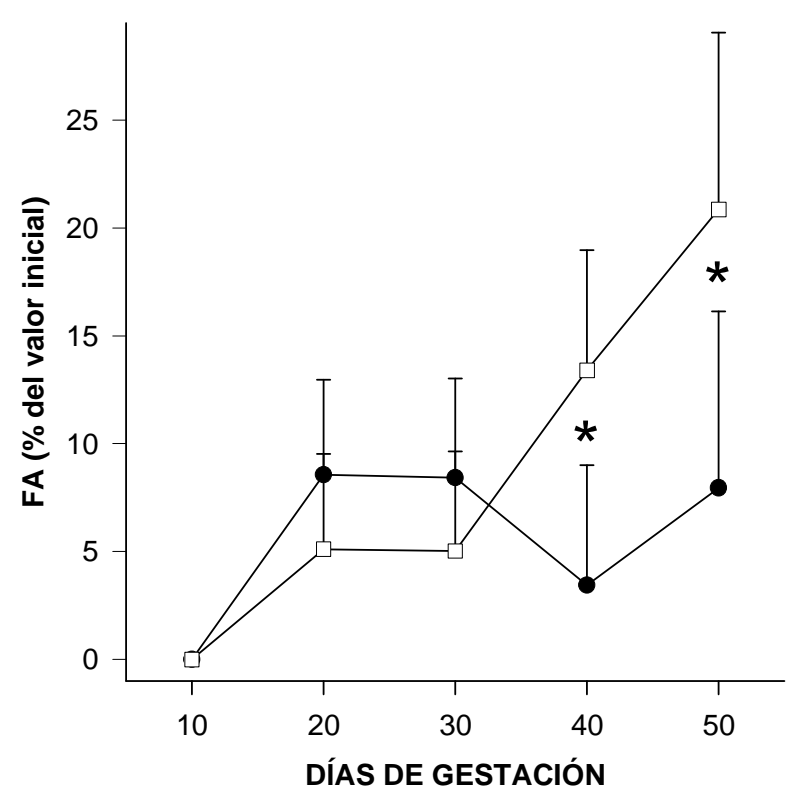

Figura 2: Porcentaje de cambio de la fracción de acortamiento (FA; media $\pm E E)$ de los mismos grupos de animales y experimento de la Figura 1.

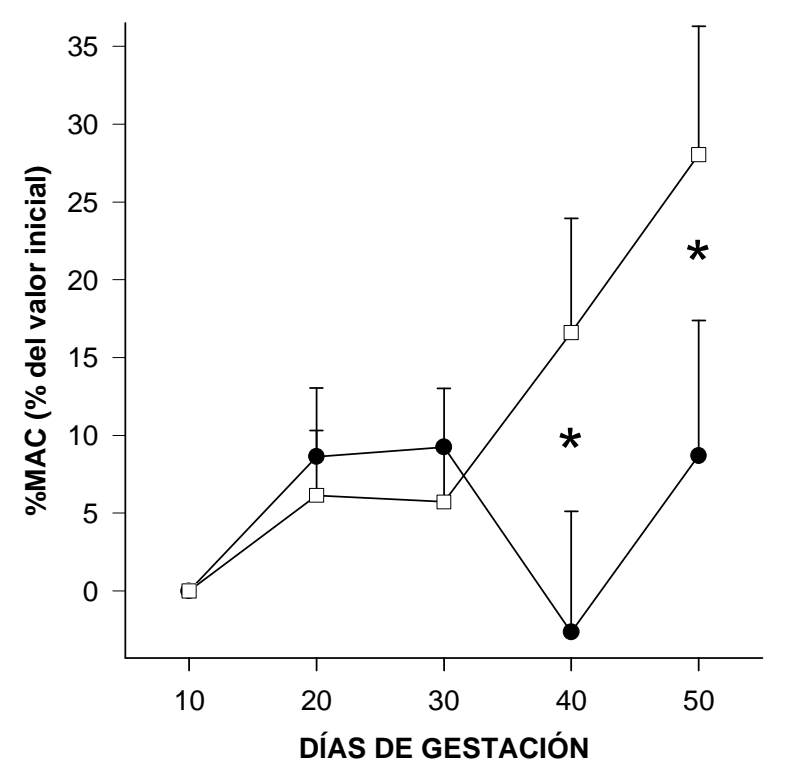

Figura 3: Porcentaje de cambio del porcentaje medio de acortamiento circunferencial (\% MAC; media $\pm E E)$ de los mismos grupos de animales y experimento de la Figura 1. 


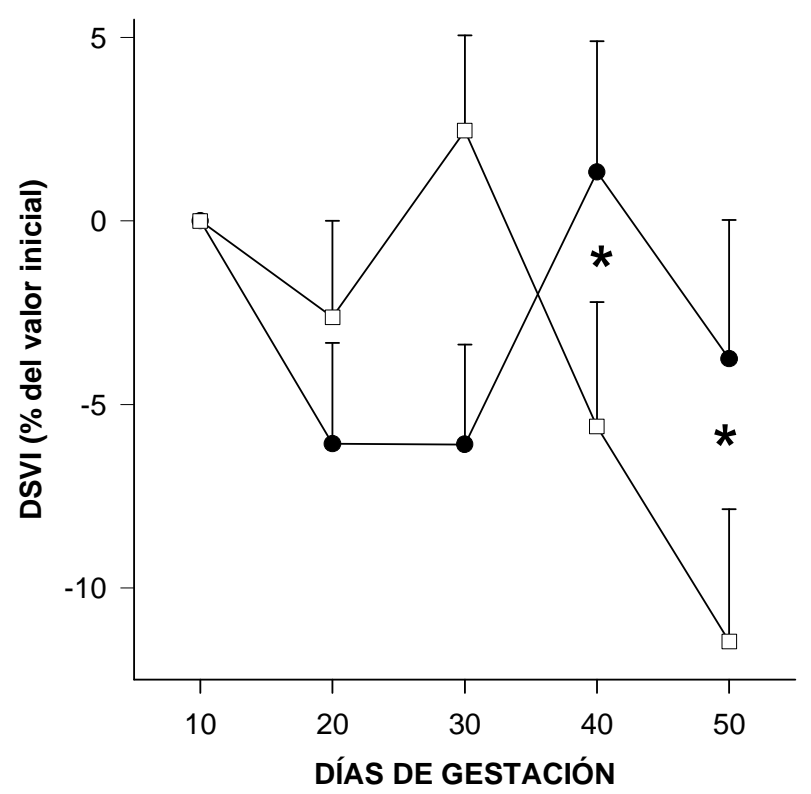

Figura 4: Porcentaje de cambio del diámetro sistólico del VI (DSVI; media $\pm E E)$ de los mismos grupos de animales y experimento de la Figura 1.

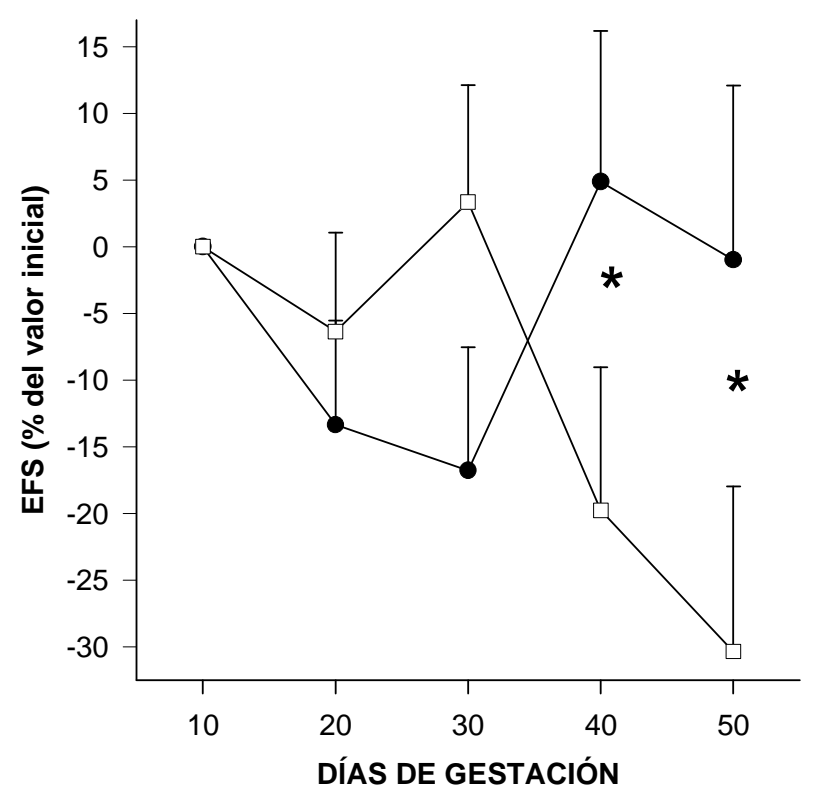

Figura 5: Porcentaje de cambio del estrés de fin de sístole (EFS; media $\pm E E)$ de los mismos grupos de animales y experimento de la Figura 1. 


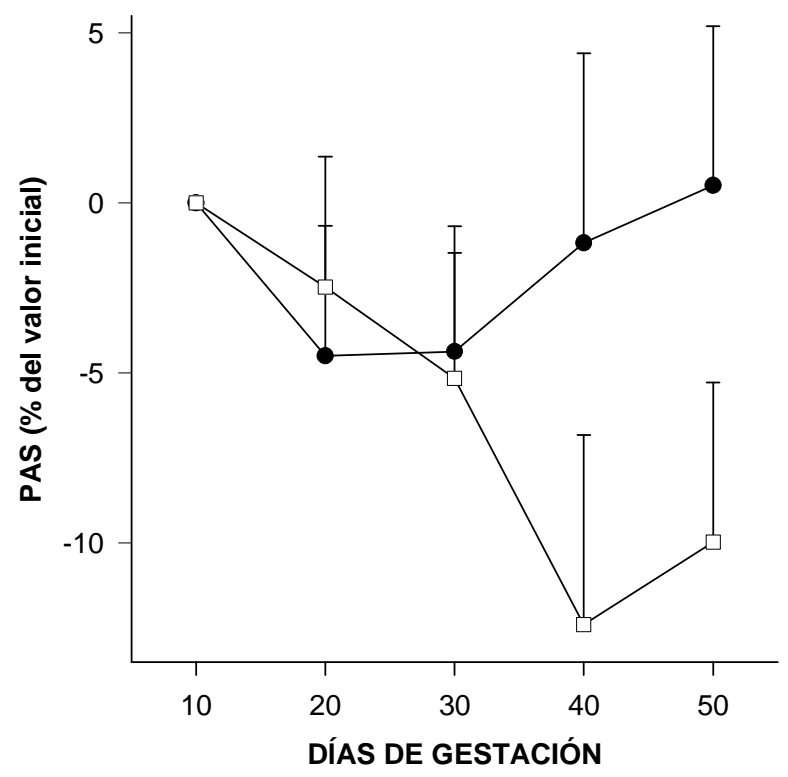

Figura 6: Porcentaje de cambio de la presión arterial sistémica (PAS; media $\pm E E)$ de los mismos grupos de animales y experimento de la Figura 1. 
Tabla 1. Porcentaje de cambio de parámetros ecocardiográficos (media $\pm E E$ ) de 10 perras preñadas anormales (GA) y 10 normales (GN) evaluadas cada 10 días desde el día 10 al 50 de gestación.

\begin{tabular}{|c|c|c|c|c|}
\hline \multirow{2}{*}{ Parámetros } & \multicolumn{4}{|c|}{ Días de gestación } \\
\hline & 20 & 30 & 40 & 50 \\
\hline \multicolumn{5}{|l|}{ TIVD } \\
\hline GA & $2,1 \pm 4,2$ & $-6,0 \pm 3,3$ & $-0,4 \pm 5,5$ & $-5,8 \pm 5,5$ \\
\hline GN & $6,8 \pm 4,2$ & $9,1 \pm 3,3$ & $2,0 \pm 5,5$ & $1,4 \pm 5,5$ \\
\hline \multicolumn{5}{|l|}{ TIVS } \\
\hline GA & $-1,3 \pm 5,3$ & $2,4 \pm 4,3$ & $1,8 \pm 5,5$ & $-6,6 \pm 5,5$ \\
\hline GN & $4,0 \pm 5,3$ & $3,5 \pm 4,3$ & $0,3 \pm 5,5$ & $1,6 \pm 5,5$ \\
\hline \multicolumn{5}{|l|}{ DDVI } \\
\hline GA & $-3,3 \pm 1,8$ & $-2,5 \pm 2,5$ & $3,2 \pm 2,2$ & $0,0 \pm 2,3$ \\
\hline GN & $-1,4 \pm 1,8$ & $3,6 \pm 2,4$ & $0,2 \pm 2,1$ & $-3,6 \pm 2,2$ \\
\hline \multicolumn{5}{|l|}{ PPD } \\
\hline GA & $-2,0 \pm 5,3$ & $1,1 \pm 5,0$ & $-5,7 \pm 6,2$ & $-6,6 \pm 5,4$ \\
\hline GN & $-6,1 \pm 5,3$ & $-4,1 \pm 5,0$ & $-6,7 \pm 6,2$ & $4,1 \pm 5,4$ \\
\hline \multicolumn{5}{|l|}{ MV } \\
\hline GA & $-5,8 \pm 4,7$ & $-9,5 \pm 5,7$ & $-2,2 \pm 5,1$ & $-9,8 \pm 6,3$ \\
\hline GN & $-1,1 \pm 4,7$ & $11,1 \pm 5,7$ & $-1,3 \pm 5,1$ & $-1,6 \pm 6,3$ \\
\hline \multicolumn{5}{|l|}{ RRE } \\
\hline GA & $1,7 \pm 6,8$ & $3,7 \pm 5,0$ & $-7,1 \pm 6,9$ & $-6,2 \pm 6,3$ \\
\hline GN & $-5,1 \pm 6,5$ & $-8,3 \pm 4,8$ & $-7,0 \pm 6,6$ & $8,1 \pm 6,0$ \\
\hline \multicolumn{5}{|l|}{$\mathrm{Ao} / \mathrm{AI}$} \\
\hline GA & $1,2 \pm 5,2$ & $-6,2 \pm 4,1$ & $-1,9 \pm 4,2$ & $-2,4 \pm 4,1$ \\
\hline GN & $3,7 \pm 5,2$ & $8,8 \pm 4,1$ & $3,5 \pm 4,2$ & $5,7 \pm 4,1$ \\
\hline \multicolumn{5}{|l|}{$\mathrm{PE}$} \\
\hline GA & $3,1 \pm 1,8$ & $1,7 \pm 2,6$ & $0,5 \pm 1,4$ & $5,2 \pm 2,5$ \\
\hline GN & $3,7 \pm 1,8$ & $-1,2 \pm 2,6$ & $0,5 \pm 1,4$ & $-1,0 \pm 2,5$ \\
\hline \multicolumn{5}{|l|}{$\mathrm{AA}$} \\
\hline GA & $1,3 \pm 6,4$ & $5,5 \pm 3,9$ & $17,4 \pm 6,5$ & $9,6 \pm 5,9$ \\
\hline GN & $4,3 \pm 6,7$ & $-10,2 \pm 4,2$ & $-4,3 \pm 6,9$ & $-11,7 \pm 6,2$ \\
\hline \multicolumn{5}{|l|}{ PSSE } \\
\hline GA & $13,5 \pm 7,0$ & $31,7 \pm 7,7$ & $54,8 \pm 15,9$ & $51,4 \pm 17,2$ \\
\hline GN & $6,5 \pm 7,0$ & $20,2 \pm 7,7$ & $24,2 \pm 15,9$ & $5,2 \pm 17,2$ \\
\hline \multicolumn{5}{|l|}{ VS } \\
\hline GA & $5,2 \pm 4,4$ & $12,3 \pm 6,4$ & $10,6 \pm 5,1$ & $12,1 \pm 5,3$ \\
\hline GN & $0,9 \pm 4,4$ & $9,7 \pm 6,4$ & $6,2 \pm 5,1$ & $1,1 \pm 5,3$ \\
\hline \multicolumn{5}{|l|}{ GC } \\
\hline GA & $13,3 \pm 9,9$ & $14,9 \pm 11,2$ & $15,8 \pm 12,7$ & $22,3 \pm 11,7$ \\
\hline GN & $-2,6 \pm 9,9$ & $12,8 \pm 11,2$ & $16,0 \pm 12,7$ & $5,3 \pm 11,7$ \\
\hline \multicolumn{5}{|l|}{$\mathrm{FC}$} \\
\hline GA & $8,6 \pm 6,6$ & $-6,1 \pm 4,6$ & $7,0 \pm 5,6$ & $6,3 \pm 6,5$ \\
\hline GN & $0,2 \pm 6,6$ & $4,5 \pm 4,6$ & $10,6 \pm 5,6$ & $14,5 \pm 6,5$ \\
\hline
\end{tabular}


TIVD $=$ Tabique interventricular en diástole; TIVS = Tabique interventricular en sístole; DDVI = Diámetro diastólico de VI; PPD = Pared posterior en diástole $;$ MV = Masa ventricular; $\mathrm{RRE}=$ Relación radio espesor; Ao/AI = Relación Ao/AI; PE = Período eyectivo; AA = Amplitud aórtica; PSSE = Punto de separación septal E; VS = Volumen sistólico $;$ GC = Gasto cardíaco $; \mathrm{FC}=$ Frecuencia cardíaca

\section{Discusión}

En este estudio, las perras con gestación normal se caracterizaron por una hipertrofia cardíaca y un aumento en la función sistólica. Estos hallazgos están en línea con reportes previos durante la gestación canina fisiológica, donde las paredes ventriculares se engrosan, mientras que el DDVI crece y el DSVI disminuye (4,5). Aunque las perras normales de este trabajo incrementaron el espesor de la PPS, este parámetro permaneció constante en los casos patológicos. Estos resultados también están en línea con lo reportado por Novelli y col. (2003; 23), quienes describieron diferencias morfológicas cardíacas entre el embarazo anormal y el resultado obstétrico favorable en mujeres (23).

La FA y el \% MAC aumentaron durante la gestación canina normal $(5,24)$, pero no lo hicieron en el grupo patológico. Este resultado también explicaría la diferencia en el DSVI, cuyo descenso normalmente provoca un incremento en la FA (18). Estos resultados están en línea con los de medicina humana, donde se midió la fracción de eyección para evaluar la función sistólica en el embarazo anormal (23). Este último estudio describió un aumento significativo en la fracción de eyección en las gestaciones normales pero no en las complicadas, indicando una respuesta cardiovascular alterada a la sobrecarga de volumen que 
caracteriza al embarazo. También se ha demostrado que la función sistólica materna se altera en las gestaciones complicadas con hipertensión desde el primer tercio $(10,25)$.

El descenso en EFS, encontrado en las perras normales pero no en las anormales, coincide con lo descripto para esta especie anteriormente (5) y con reportes en humanos, donde este parámetro es más alto en las embarazadas hipertensas que en las normales (25). Es sabido que el EFS es una medida de función miocárdica calculada a partir de la PAS (19). Por ende en los casos anormales, la falta de descenso en el EFS podría deberse a la falla de la PAS para disminuir o a una función sistólica deficiente.

Como era de esperar, la PAS decreció progresivamente a través del estudio en el grupo normal $(5,10)$. Sin embargo, no se observó esta disminución en las perras que subsecuentemente desarrollaron un desenlace gestacional anormal. Una situación similar se presenta en mujeres con preeclampsia, un desorden hipertensivo del embarazo que se correlaciona altamente con una pobre perfusión uterina y una desenlace gestacional adverso $(1,7,26)$. Cuatro de las perras patológicas tuvieron valores de PAS de 150 a $160 \mathrm{~mm} \mathrm{Hg}$ durante la segunda mitad de su preñez. En perros y gatos, estos valores de PAS se asocian con un riesgo leve a moderado de daño en órganos blanco (27). Sería interesante en el futuro, realizar un seguimiento y estudio de estos casos para establecer si, como en las mujeres, existe también un cuadro de preeclampsia subclínica en perros (9). Aunque no se encontraron diferencias significativas en la FC, este parámetro fue ligeramente más alto en las hembras normales, lo cual podría indicar una falta de adaptación hemodinámica en la gestación anormal (28).

Las disimilitudes cardiovasculares en las perras preñadas podrían ser utilizadas para identificar en forma confiable los casos patológicos. Así, la evaluación ecocardiográfica y 
Doppler de la morfología y función cardíacas podría constituir un método no invasivo válido que contribuya al monitoreo de la salud materna y fetal.

En el presente estudio, la adaptación cardiovascular anormal parece predecir la aparición de complicaciones. Conocer los mecanismos cardíacos y vasculares probablemente involucrados en la mala adaptación materna podría también ser de utilidad en el manejo de pacientes en la fase preclínica de la enfermedad.

Concluimos que los signos de una adaptación cardiovascular materna alterada durante la gestación pueden convertirse en predictores de complicaciones obstétricas en perros, antes de la aparición clínica de enfermedad materna y fetal. Además, la información derivada de la hemodinamia materna podría ser útil a la hora de decidir un tratamiento clínico.

\section{Bibliografía}

1. Valensise H, Novelli GP, Vasapollo B, Borzi M, Arduini D, Galante A, Romanini C. Maternal cardiac systolic and diastolic function: relationship with uteroplacental resistances. A Doppler and echocardiographic longitudinal study. Ultrasound Obstet Gynecol 2000;15:487-97.

2. Eghbali M, Deva R, Alioua A, Minosyan TY, Ruan H, Wang Y, Toro L, Stefani E. Molecular and Functional Signature of Heart Hypertrophy During Pregnancy. Circ Res 2005;96:1208-16.

3. Blanco PG, Arias DO, Gobello C. Doppler ultrasound in canine pregnancy. J Ultrasound Med 2008;27:1745-50. 
4. Williams JG, Rincon-Skinner T, Sun D, Wang Z, Zhang S, Zhang X, Hintze TH. Role of nitric oxide in the coupling of myocardial oxygen consumption and coronary vascular dynamics during pregnancy in the dog. AmJPhysiol HeartCirc Physiol 2007;293:H2479-86. 5. Blanco PG, Tórtora M, Rodríguez R, Arias D, Gobello C. Ultrasonographic assessment of maternal cardiac function and peripheral circulation during normal gestation in dogs. Vet $\mathbf{J}$ 2010; doi:10.1016/j.tvj1.2010.08.013.

6. Spotswood TC, Kirberger RM, Koma LM, Thompson PN, Miller DB. Changes in echocardiographic variables of left ventricular size and function in a model of canine normovolemic anemia. Vet Radiol Ultrasound 2006;47:358-65.

7. Easterling TR, Watts DH, Schmucker BC, Benedetti TJ. Measurement of cardiac output during pregnancy: validation of Doppler technique and clinical observations in preeclampsia. Obstet Gynecol 1987;69:845-50.

8. Novelli GP, Valensise H, Vasapollo B, Larciprete G, Altomare F, Di Pierro G, Casalino B, Galante A, Arduini D. Left ventricular concentric geometry as a risk factor in gestational hypertension. Hypertension 2003;41:469-75.

9. Vasapollo B, Novelli GP, Valensise H. Total vascular resistance and left ventricular morphology as screening tools for complications in pregnancy. Hypertension 2008;51: 10206.

10. Kametas NA, McAuliffe F, Hancock J, Chambers J, Nicolaides KH. Maternal left ventricular mass and diastolic function during pregnancy. Ultrasound Obstet Gynecol 2001;18:460-6.

11. Blanco PG, Arias D, Rube A, Barrena JP, Corrada Y, Gobello C. An experimental model to study resistance index and systolic/diastolic ratio of uterine arteries in adverse canine pregnancy outcome. Reprod Domest Anim 2009;44:164-6. 
12. Davidson AP. Approaches to Reducing Neonatal Mortality in Dogs. En: Concannon PW, England G, Verstegen J, Linde-Forsberg C, editores. Recent Advances in Small Animal Reproduction. Ithaca, New York, IVIS, 2003; www.ivis.org. Document number A1226.0303. 13. Münnich A. The pathological newborn in small animals: the neonate is not a small adult. Vet Res Commun 2008;32:S81-5.

14. Olson PN, Husted PW, Allen TA, Nett TM. Reproductive endocrinology and physiology of the bitch and queen. Vet Clin North Am Small Anim Pract 1984;14:927-46.

15. Chetboul V, Tidholm A, Nicolle A, Sampedrano CC, Gouni V, Pouchelon JL, Lefebvre HP, Concordet D. Effects of animal position and number of repeated measurements on selected two-dimensional and M-mode echocardiographic variables in healthy dogs. J Am Vet Med A 2005;227:743-7.

16. Thomas WP, Gaber CE, Jacobs GJ, Kaplan PM, Lombard CW, Moise NS, Moses BL. Recommendations for standards in transthoracic two-dimensional echocardiography in the dog and cat. Echocardiography Committee of the Specialty of Cardiology, American College of Veterinary Internal Medicine. J Vet Intern Med 1993;7:247-52.

17. Kienle RD. Echocardiography. En: Kittelson MD, Kienle RD, editores. Small animal cardiovascular medicine textbook. St. Louis, Mosby; 1998. p. 95-117.

18. Boon J. The echocardiographyc examination. En: Boon JA, editora. Manual of Veterinary Echocardiography. Media, Williams and Wilkins; 1998. p. 35-128.

19. Vuille C, Weyman AE. Left ventricle I: general considerations, assessment of chamber size and function. En: Weyman AE, editor. Principles and practice of echocardiography. Media, Williams and Wilkins;1994. p. 575-624.

20. Estrada A, Maisenbacher H. Calculation of stenotic valve area. J Vet Cardiol 2006;8:4953. 
21. Gottdiener JS, Bednarz J, Devereux R, Gardin J, Klein A, Manning WJ, Morehead A, Kitzman D, Oh J, Quinones M, Schiller NB, Stein JH, Weissman NJ. American Society of Echocardiography recommendations for use of echocardiography in clinical trials. J Am Soc Echocardiog 2004;17:1086-119.

22. Littman MP, Fox PR. Systemic hypertension: recognition and treatment. En: Fox P, Sisson D, Moïse NS, editores. Textbook of canine and feline cardiology. Philadelphia, WB Saunders Co;1999. p. 795-813.

23. Novelli GP, Valensise H, Vasapollo B, Larciprete G, Altomare F, Di Pierro G, Casalino B, Galante A, Arduini D. Left ventricular concentric geometry as a risk factor in gestational hypertension. Hypertension 2003;41:469-75.

24. Abbott JA. The effect of pregnancy on echocardiographic variables in healthy bitches. J Vet Cardiol 2010;12:123-8.

25. Simmons L, Gillin AG, Jeremy RW. Structural and functional changes in left ventricle during normotensive and preeclamptic pregnancy. Am J Physiol Heart Circ Physiol 2002;283:H1627-33.

26. Voss A, Baumert M, Baier V, Stepan H, Walther T, Faber R. Autonomic cardiovascular control in pregnancies with abnormal uterine perfusion. Am J Hypertens 2006;19:306-12. 27. Brown S, Atkins C, Bagley R, Carr A, Cowgill L, Davidson M, Egner B, Elliott J, Henik R, Labato M, Littman M, Polzin D, Ross L, Snyder P, Stepien R; American College of Veterinary Internal Medicine. Guidelines for the identification, evaluation and management of systemic hypertension in dogs and cats. Journal of Vetarinary Internal Medicine 2007;21:54258. 
28. Olsson K, Lagerstedt AS, Bergström A, Häggström J. Change of diurnal heart rate patterns during pregnancy and lactation in dogs (Canis familiaris). Acta Vet Scand 2003;4:105-10. 


\section{CAPÍtULLO V}

\section{CAMBIOS ELECTROCARDIOGRÁFICOS EN LA GESTACIÓN \\ CANINA NORMAL Y ANORMAL}

\section{Introducción}

La gestación canina es un estado físiológico caracterizado por cambios adaptativos importantes, muchos de los cuales ocurren en el sistema cardiovascular. Estos cambios incluyen un aumento en la volemia y el gasto cardíaco (GC), asociado a un hipertrofia miocárdica de tipo excéntrica $(1,2)$. Más aún, la resistencia vascular periférica está disminuida y el flujo sanguíneo uterino aumentado (3).

Es probable que estos cambios anatómicos y funcionales modifiquen a su vez el sistema de conducción cardíaca. Otros factores, incluyendo las variaciones en los niveles de hormonas circulantes, los cambios en el tono autonómico y los niveles de electrolitos pueden también contribuir con estas modificaciones $(4,5)$.

La remodelación eléctrica, que resulta de los cambios morfológicos y de la expresión génica, se asocia particularmente con una prolongación del potencial de acción. Este fenómeno puede ser consecuencia de una reducción en la corriente de salida del potasio intracelular (6). Por este motivo, se ha reportado un aumento en la duración del intervalo QT en el transcurso de la gestación (7).

La ausencia de estos mecanismos adaptativos del embarazo normal puede poner en riesgo la salud de la madre y el feto. Las mujeres embarazadas con una perfusión uterina deficiente y un consecuente resultado obstétrico patológico, muestran alteraciones en la FC y el intervalo 
QT que preceden a los síntomas clínicos. Adicionalmente, en la mujer existen evidencias que sugieren que la gestación puede exacerbar arritmias preexistentes y subyacentes o incluso causar arritmias nuevas (5).

La evaluación electrocardiográfica se ha realizado en el curso de la preñez en las perras, para registrar la FC materna, demostrando un incremento de este parámetro durante el período gestacional $(3,8,9)$. Mientras existe abundante información sobre los cambios estructurales y la FC, se sabe muy poco acerca de otras alteraciones electrofisiológicas cardíacas durante la gestación canina normal. Más aún en esta especie, el monitoreo electrocardiográfico no se ha descripto durante las gestaciones patológicas. Es importante destacar que algunas de las enfermedades que causan aborto o muerte perinatal, también pueden provocar una afección sistémica que desencadene un malfuncionamiento cardíaco (10). En estos casos, las modificaciones electrocardiográficas del trazado normal pueden aparecer antes que se desarrollen los signos sistémicos u obstétricos (11).

La comprensión más detallada de la electrofisiología durante la preñez es esencial para maximizar su uso en el monitoreo de la salud materna y fetal. Por todo esto, el objetivo de este estudio fue analizar los cambios electrocardiográficos en el curso de la gestación normal y anormal en las hembras caninas. 


\section{Materiales y métodos}

Animales y seguimiento

Para este experimento se incluyeron 33 perras puras y mestizas, de 1-8 años de edad, de 3-55 kg de peso. Todas las hembras fueran negativas a Brucella canis y clasificadas retrospectivamente como perras preñadas normales $(n=12)$ o anormales $(n=11)$. Además, se incluyó un grupo control de perras en diestro no preñadas $(\mathrm{n}=10)$. Las hembras normales parieron cachorros sanos a término, mientras que las anormales interrumpieron su preñez entre el día 52 y 60 (desde el día estimado de pico de LH) o presentaron muerte perinatal mayor al $60 \%$ de la camada $(12,13)$. Las perras se evaluaron electrocardiográficamente cada 10 días del día 0 (día estimado del pico de LH) hasta el día 65 del ciclo estral, el parto o el aborto. Este estudio fue aprobado por el Comité de Cuidado y Uso Animal de esta Institución.

\section{Electrocardiogramas}

Para realizar el electrocardiograma (ECG), los pacientes se posicionaron en decúbito lateral derecho sobre una camilla cubierta de goma. Las perras se sujetaron suavemente con los cuatro miembros sostenidos perpendiculares al cuerpo y apenas separados (14). Los electrodos se acoplaron a la piel con clips tipo cocodrilos metálicos que se humedecieron con alcohol para mejorar la transmisión eléctrica.

Las derivaciones I, II, III, $\mathrm{aV}_{\mathrm{R}}, \mathrm{aV}_{\mathrm{L}}, \mathrm{aV}_{\mathrm{F}}, \mathrm{CV}_{6 \mathrm{LU}}, \mathrm{CV}_{6 \mathrm{LL}}, \mathrm{CV}_{5 \mathrm{RL}}$ y $\mathrm{V}_{10}$ se registraron en papel calibrado a una velocidad de $50 \mathrm{~mm} / \mathrm{s}$, como ha sido previamente reportado (15). La derivación II se registró también a una velocidad de $25 \mathrm{~mm} / \mathrm{s}$ para calcular la FC y ritmo 
cardíaco (RC; ritmo sinusal o arritmia sinusal). Luego del registro, un único operador entrenado se encargó de analizar cada ECG. El eje eléctrico medio (EEM), amplitud de onda P (Pa), duración de onda P (Pd), intervalo P-R (PR), amplitud del complejo QRS (QRSa), duración del complejo QRS (QRSd), intervalo Q-T (QT), segmento S-T (ST; mv) y onda T ( $\mathrm{T}$; menor o mayor al 25\% de amplitud de la onda $\mathrm{R}$ ) se calcularon manualmente a una velocidad de papel de $50 \mathrm{~mm} / \mathrm{s}$ (16). Se promediaron tres latidos y seis latidos en caso de arritmia sinusal. Se registró el intervalo RR inmediatamente precedente a cada complejo y el intervalo QT fue corregido (QTc) por la fórmula de Van de Water (QTc = QT-0.087(RR1000); 17).

\section{Análisis estadístico}

La FC, EEM, Pa, Pd, PR, QRSa, QRSd, QT y QTc se transformaron a porcentaje de cambio ([Valor final -valor inicial / valor inicial] x 100) para evitar el efecto de los diferentes pesos corporales. Las comparaciones entre los grupos se efectuaron con ANOVA de medidas repetidas seguido del test de Tukey. El ritmo cardíaco y la onda T fueron analizados con una prueba de Chi cuadrado (SPSS 17.0, SPSS Inc. Chicago, IL, USA).

\section{Resultados}

No hubo diferencias en peso $(p>0,1)$ ni edad $(p>0,1)$ entre los grupos. El tamaño de camada tampoco difirió entre los grupos de perras preñadas $(\mathrm{p}>0,1)$. Ninguno de los animales presentó arritmias severas durante el experimento. Los porcentajes de cambio de la FC (p < 
0,01), Pa (p<0,01), QRSa ( $p<0,01)$, QRSd $(\mathrm{p}<0,05)$ y QTc $(\mathrm{p}<0,05)$ se modificaron de diferente manera a lo largo del tiempo en los tres grupos.

En la gestación normal, el porcentaje de cambio de la FC se incrementó progresivamente desde el día 40 al 60, 31,3\% vs. -1,8\% en perras en diestro (Figura 1). Por el contrario, este parámetro no mostró el mismo incremento en el grupo anormal (Figura 1). Al día 50 de gestación, el porcentaje de cambio de la Pa disminuyó hasta un -20,1\% y -17,8\% en la preñez normal y anormal, respectivamente, mientras que no se observó ninguna disminución en los animales en diestro (Tabla 1). En el grupo de preñez anormal, el porcentaje de cambio de la QRSa cayó a -34\% al día 60 (Figura 2), pero esto no sucedió en los otros grupos. A la misma edad gestacional, el porcentaje de cambio de la QRSd fue 6,2\% vs. -4,9\% en la gestación normal y el diestro, respectivamente (Tabla 1). En el grupo de preñez normal, el porcentaje de cambio del QTc aumentó desde el día 40 en adelante hasta un 9,9\% vs. 4,3\% en las perras en diestro, mientras que este parámetro permaneció constante en las hembras anormales (Figura $3)$.

Al día 60 de gestación, la mayor parte de las hembras preñadas de ambos grupos presentaron un ritmo sinusal normal en comparación con las perras en diestro, las cuales desarrollaron en su mayoría arritmia sinusal ( $\mathrm{p}<0,05$; Figura 4). Los porcentajes de cambio del EEM, Pd, PR y QT tanto como el ST y la onda T no difirieron entre los grupos (Tabla 2 y Tabla 3). 


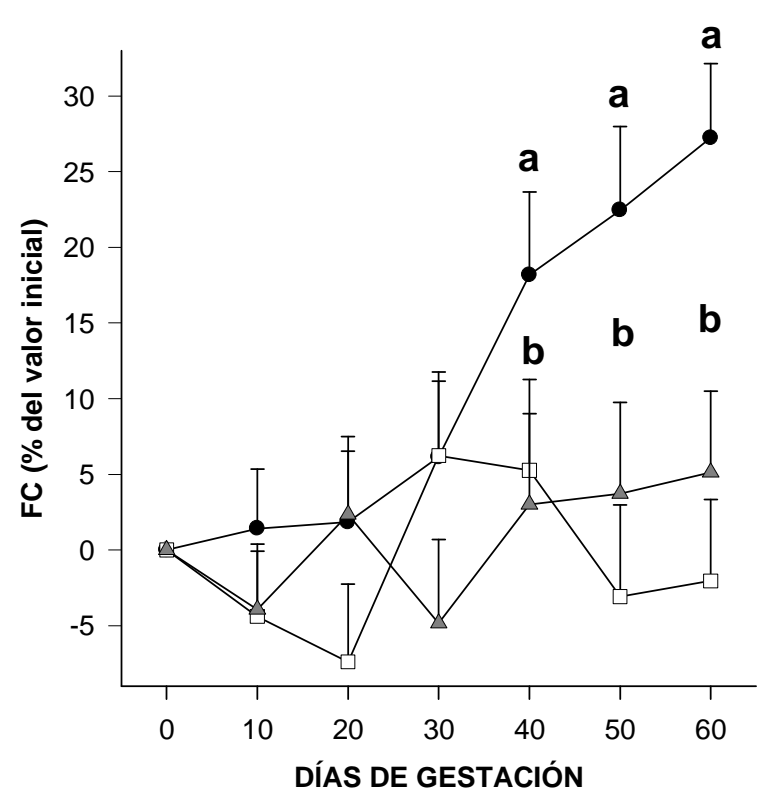

Figura 1. Porcentaje de cambio de la FC (media \pm EE) de 12 perras preñadas normales (GN; círculos), 11 perras preñadas anormales (GA; triángulos) y 10 perras vacías en diestro (D; cuadrados) evaluadas cada 10 días desde el pico estimado de LH. Las letras a y b indican diferencias post-hoc ( $p<0,01)$ entre GN vs. D y GN vs. GA, respectivamente.

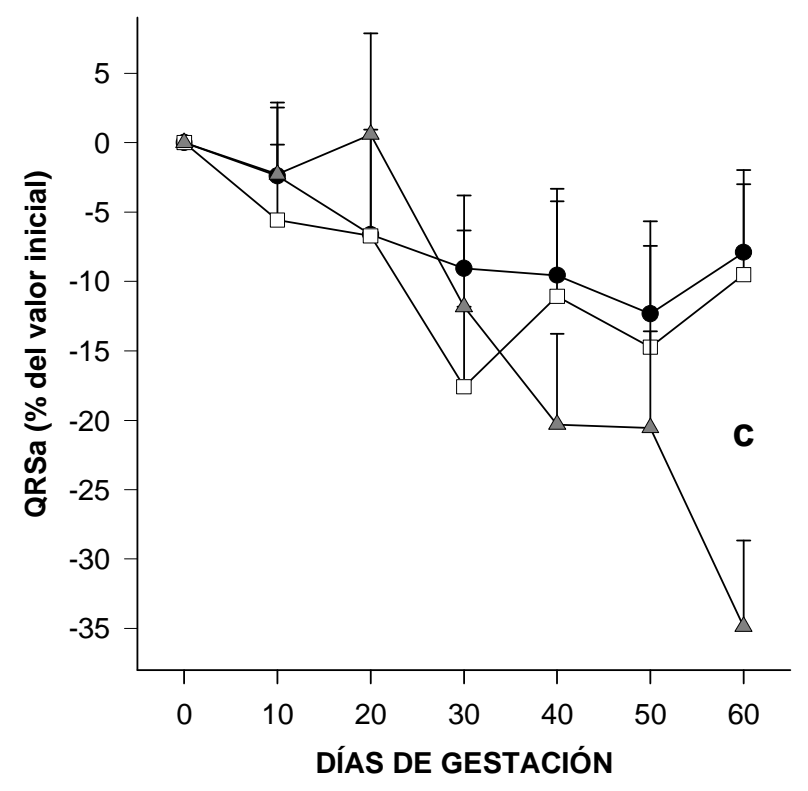

Figura 2. Porcentaje de cambio de la amplitud del QRS (QRSa; media $\pm E E)$ de las mismas perras y experimento que la Figura 1. La letra $\mathrm{c}$ indica diferencias post-hoc $(\mathrm{p}<0,01)$ entre GN y D vs. GA. 


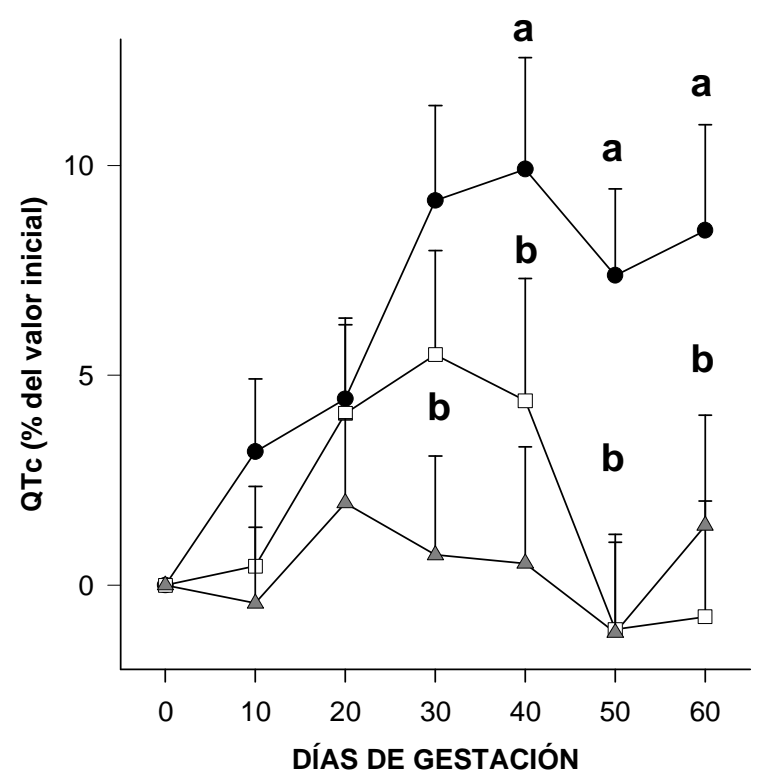

Figura 3. Porcentaje de cambio del intervalo QT corregido (QTc; media $\pm E E$ ) de las mismas perras y experimento que la Figura 1. Las letras a y b indican diferencias post-hoc $(\mathrm{p}<0,05)$ entre GN vs. D y GN vs. GA, respectivamente.

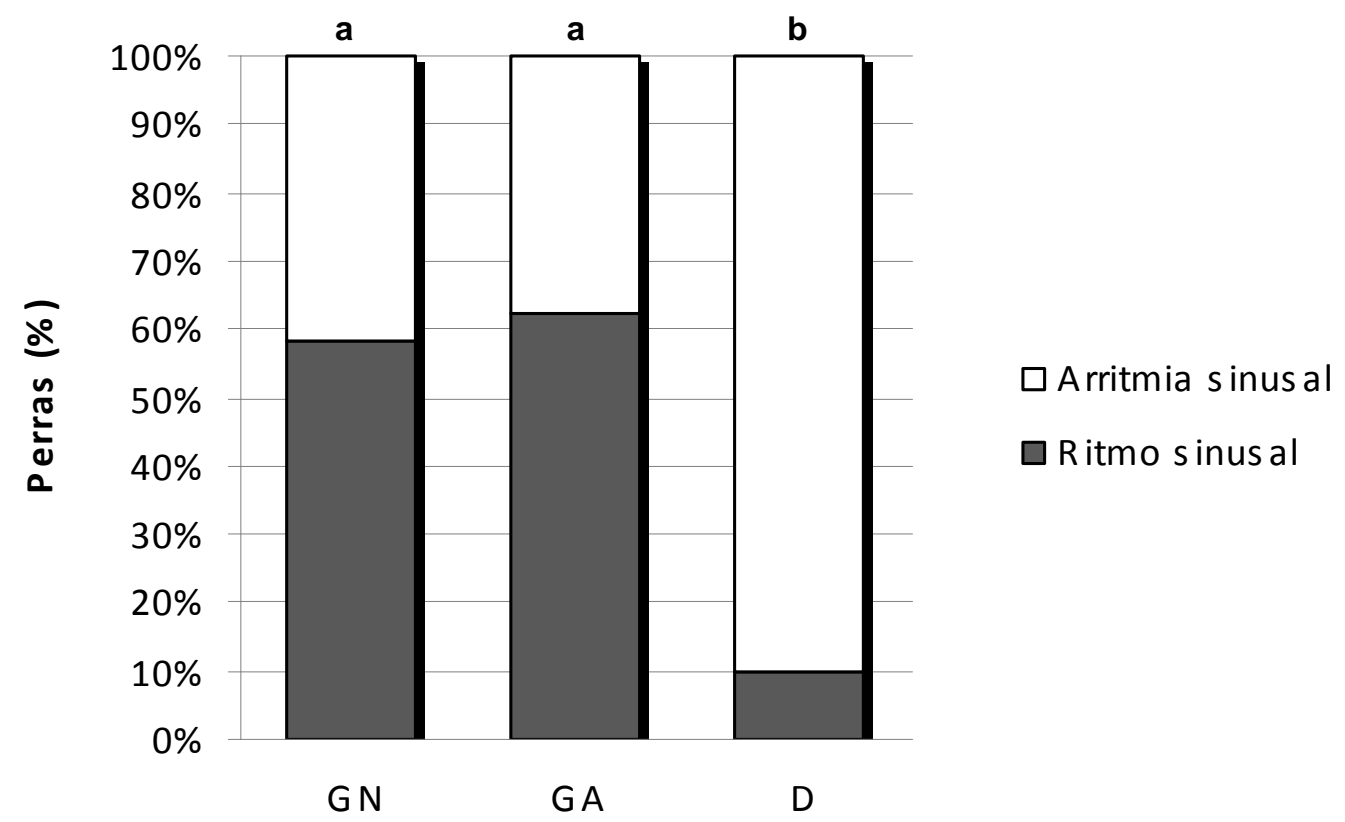

Figura 4. Porcentaje de perras de la Figura 1 al día 60 del estudio con diferentes ritmos cardíacos. Las diferentes letras sobre las columnas indican diferencias de $\mathrm{p}<0,05$. 
Tabla 1: Porcentaje de cambio de la amplitud de onda $\mathrm{P}(\mathrm{Pa}$; media $\pm \mathrm{EE})$ y la duración del QRS (QRSd; media \pm EE) de 12 perras preñadas normales (GN), 11 perras preñadas anormales (GA) y 10 perras vacías en diestro (D) evaluadas cada 10 días desde el pico estimado de LH.

\begin{tabular}{|c|c|c|c|c|c|c|}
\hline \multirow{2}{*}{ Parámetro } & \multicolumn{7}{|c|}{ Días de gestación } \\
\cline { 2 - 7 } & 10 & 20 & 30 & 40 & 50 & 60 \\
\hline Pa & $0,4 \pm 8$ & $-18,7 \pm 9$ & $-15,9 \pm 9$ & $-5,5 \pm 10$ & $-20,1 \pm 10^{\mathrm{b}}$ & $-9,7 \pm 7$ \\
\hline GN & $-7,1 \pm 8$ & $7,7 \pm 9$ & $-1,6 \pm 10$ & $-9,6 \pm 10$ & $-17,8 \pm 10^{\mathrm{b}}$ & $-30,9 \pm 8$ \\
\hline GA & $1,6 \pm 8$ & $-3,3 \pm 10$ & $-8,3 \pm 10$ & $-1,6 \pm 11$ & $1,6 \pm 11^{\mathrm{a}}$ & $-6,6 \pm 8$ \\
\hline D & $0,5 \pm 3$ & $-4,4 \pm 3$ & $-0,2 \pm 4$ & $-0,9 \pm 5$ & $3,8 \pm 5$ & $6,2 \pm 4^{\mathrm{b}}$ \\
\hline QRSd & $2,2 \pm 3$ & $-6,0 \pm 3$ & $4,6 \pm 5$ & $-4,8 \pm 6$ & $3,0 \pm 6$ & $0,7 \pm 4^{\mathrm{b}}$ \\
\hline GN & $0,0 \pm 4$ & $8,9 \pm 3$ & $7,5 \pm 4$ & $1,0 \pm 6$ & $1,0 \pm 6$ & $-4,9 \pm 5^{\mathrm{a}}$ \\
\hline GA
\end{tabular}

Los diferentes superíndices dentro de una misma columna indican diferencias $(\mathrm{p}<0,05)$ entre grupos. 
Tabla 2: Porcentaje de cambio (media土EE) del eje eléctrico medio (EEM), duración de onda P (Pd), intervalo P-R e intervalo QT (QT) de las mismas perras y experimento de la Tabla $\mathbf{1}$.

\begin{tabular}{|c|c|c|c|c|c|c|}
\hline \multirow{2}{*}{ Parámetro } & \multicolumn{6}{|c|}{ Días de gestación } \\
\cline { 2 - 7 } & 10 & 20 & 30 & 40 & 50 & 60 \\
\hline MEA & \multicolumn{7}{|c|}{} \\
\hline GN & $34,9 \pm 15$ & $16,7 \pm 13$ & $18,9 \pm 18$ & $7,9 \pm 16$ & $80,7 \pm 48$ & $43,4 \pm 31$ \\
\hline GA & $7,0 \pm 15$ & $12,4 \pm 13$ & $-0,7 \pm 19$ & $-0,2 \pm 17$ & $-40,9 \pm 51$ & $-72,9 \pm 33$ \\
\hline D & $13,4 \pm 16$ & $8,2 \pm 14$ & $7,9 \pm 20$ & $19,7 \pm 18$ & $9,0 \pm 53$ & $16,3 \pm 34$ \\
\hline Pd & $-8,3 \pm 2$ & $-1,3 \pm 4$ & $-9,7 \pm 3$ & $-5,5 \pm 3$ & $-1,3 \pm 4$ & $-5,5 \pm 3$ \\
\hline GN & $0 \pm 2$ & $3,1 \pm 5$ & $5,6 \pm 4$ & $2,5 \pm 3$ & $2,2 \pm 4$ & $7,1 \pm 3$ \\
\hline GA & $7,5 \pm 3$ & $5,0 \pm 5$ & $0,0 \pm 4$ & $0,0 \pm 3$ & $5,0 \pm 4$ & $5,0 \pm 4$ \\
\hline D & $-0,1 \pm 2$ & $3,0 \pm 2$ & $-2,0 \pm 2$ & $0,4 \pm 3$ & $-4,6 \pm 3$ & $-6,9 \pm 3$ \\
\hline PR & $4,9 \pm 2$ & $1,8 \pm 2$ & $5,7 \pm 2$ & $3,5 \pm 3$ & $-1,2 \pm 3$ & $-2,1 \pm 3$ \\
\hline GN & $1,2 \pm 2$ & $1,7 \pm 2$ & $2,0 \pm 3$ & $4,2 \pm 3$ & $0,2 \pm 4$ & $4,1 \pm 3$ \\
\hline GA & $3,4 \pm 2$ & $5,3 \pm 2$ & $9,5 \pm 2$ & $8,2 \pm 3$ & $4,3 \pm 2$ & $4,8 \pm 2$ \\
\hline D & $-0,7 \pm 2$ & $2,1 \pm 2$ & $6,2 \pm 2$ & $-1,3 \pm 3$ & $-3,0 \pm 2$ & $-2,8 \pm 3$ \\
\hline QT & $2,5 \pm 2$ & $7,4 \pm 2$ & $5,5 \pm 3$ & $4,0 \pm 3$ & $0,0 \pm 2$ & $0,0 \pm 3$ \\
\hline GN & \multicolumn{7}{|l|}{} \\
\hline GA & \multicolumn{7}{|l|}{}
\end{tabular}

Tabla 3: Intervalo S-T (ST; media $\pm E E)$ de las mismas perras y experimento de la Tabla 1.

\begin{tabular}{|c|c|c|c|c|c|c|c|}
\hline & \multicolumn{7}{|c|}{ Días de gestación } \\
\cline { 2 - 8 } & 0 & 10 & 20 & 30 & 40 & 50 & 60 \\
\hline ST & \multicolumn{7}{|c|}{} \\
\hline GN & $-0,01 \pm 0,01$ & $0 \pm 0,01$ & $-0,01 \pm 0,05$ & $-0,01 \pm 0,05$ & $0 \pm 0,01$ & $-0,02 \pm 0,01$ & $0 \pm 0,01$ \\
\hline GA & $-0,02 \pm 0,01$ & $-0,02 \pm 0,01$ & $-0,01 \pm 0,05$ & $-0,01 \pm 0,05$ & $0 \pm 0,01$ & $0 \pm 0,01$ & $0 \pm 0,01$ \\
\hline D & $-0,06 \pm 0,01$ & $-0,04 \pm 0,01$ & $-0,04 \pm 0,05$ & $-0,02 \pm 0,05$ & $-0,03 \pm 0,01$ & $-0,05 \pm 0,01$ & $-0,05 \pm 0,01$ \\
\hline
\end{tabular}

\section{Discusión}

Según sabemos hasta el momento, este es el primer reporte de una descripción y comparación electrocardiográfica seriada durante la gestación canina normal y anormal. 
La aceleración de la FC observada en las perras normales es un fenómeno bien conocido que garantiza un incremento del GC, optimizando el aporte de oxígeno y nutrientes $(3,8,18)$. Resulta muy interesante que este mecanismo adaptativo no aparezca en las gestaciones con resultados adversos. Este hallazgo está en línea con reportes previos en mujeres, donde la FC de las pacientes con perfusión uterina deficiente es menor a la de aquellas que presentan una perfusión normal (5). Más aún en perros, enfermedades sistémicas que provocan aborto o muerte perinatal, se asocian con bradicardia materna (19).

El descenso en la $\mathrm{Pa}$ en ambos grupos de hembras preñadas puede deberse a las modificaciones electrolíticas que ocurren en el curso de la gestación. En ratones gestantes, las células miocárdicas desarrollan un descenso en el $\mathrm{I}_{\text {to-f }}$, un constituyente molecular de la corriente de salida del potasio intracelular (7). Vale la pena destacar que en la hipercalemia, el miocardio atrial canino pierde su habilidad de despolarizarse (20). Esto podría explicar el descenso de la Pa encontrado en animales preñados. Adicionalmente, durante la gestación en la mujer, se describió una conducción heterogénea y discontinuada del impulso atrial (22). El aumento de QRSd y QRSa se asocia con el agrandamiento ventricular en perros (20). Este cambio morfológico ya se ha descripto previamente en la gestación canina normal, donde la hipertrofia excéntrica aparece como un mecanismo compensatorio a la sobrecarga de volumen (3). El incremento del QRSd podría también deberse al aumento en el potasio de las células miocárdicas, el cual puede causar un potencial de acción prolongado en la gestación normal. En las mujeres, la QRSa permanece constante durante el embarazo (4). El descenso en la QRSa en el grupo anormal podría evidenciar la ausencia de la adaptación cardiovascular en estos animales. Esto coincide con reportes en mujeres con cardiomiopatía periparto, en las que con frecuencia se detecta una baja amplitud en los complejos QRS (23). Más aún, este hallazgo podría ser también un indicador temprano y subclínico de enfermedad sistémica. 
Contrariamente con reportes previos en ratones gestantes (7), el intervalo QT no se modificó durante la gestación canina normal ni anormal. Esto probablemente se deba a la aceleración materna de la FC. Esta aceleración normalmente ocurre en conjunción con un acortamiento del intervalo QT y podría enmascarar un cambio inherente en este parámetro (17).

Al ser corregido para la FC, el QTc de las perras preñadas fue más alto que el de las perras no gestantes, sugiriendo que el promedio de la duración de la despolarización y repolarización ventricular está alterada por la preñez normal. Este aumento en el QTc podría ser explicado por la prolongación del potencial de acción que resulta de la modificación en la corriente de potasio previamente descripta.

El QTc de las perras con resultados patológicos de la gestación difiere de la normalidad desde la segunda mitad de la preñez en adelante. Este resultado coincide con reportes en mujeres con desórdenes hipertensivos de la gestación, donde el QT refleja una elevada actividad nerviosa simpática (5). Es interesante destacar que en estos animales, como en las mujeres, este hallazgo electrocardiográfico apareció antes de que se dispararan los síntomas clínicos.

La corrección del QT con la FC se ha reportado en cardiología veterinaria para evaluar las arritmias ventriculares familiares de los Boxers, aunque no pareció ser una herramienta diagnóstica demasiado útil (24). Sin embargo, en nuestro trabajo, esta corrección reveló diferencias significativas entre los grupos. Se necesitan más estudios en perros sanos para determinar si esta conversión arroja información aprovechable o no.

La arritmia sinusal disminuye hacia el final de la preñez normal y anormal, sugiriendo que el tono vagal al nodo sinusal está disminuido durante la gestación (5). Esto concuerda con lo 
descripto por Lucio y col. (2009) para los períodos gestacional y periparto en las perras, donde la mayoría de las hembras tuvo un ritmo sinusal normal (9). A pesar de que el 4-14\% de los embarazos humanos normales presentan cambios inespecíficos en el ST y la onda T (25), estos parámetros no se modificaron durante la gestación canina normal ni anormal.

En este estudio, las disimilitudes cardiovasculares entre la preñez normal y anormal en las perras preceden a la aparición de complicaciones. Por este motivo, el uso de la electrocardiografía en obstetricia canina podría contribuir a identificar los desenlaces patológicos antes de que se vuelvan clínicamente evidentes.

Concluimos que durante la preñez canina, algunos parámetros electrocardiográficos comienzan a modificarse desde el día 40 y las preñeces patológicas empiezan a diferir electrocardiográficamente de la normalidad desde el día 30. Por lo tanto, el monitoreo del ECG materno durante la gestación podría ser un pilar importante en la toma de decisiones médicas en obstetricia canina.

\section{Bibliografía}

1. Williams JG, Rincon-Skinner T, Sun D, Wang Z, Zhang S, Zhang X, Hintze TH. Role of nitric oxide in the coupling of myocardial oxygen consumption and coronary vascular dynamics during pregnancy in the dog. Am J Physiol Heart Circ Physiol 2007;293:H2479-86.

2. Abbott JA. The effect of pregnancy on echocardiographic variables in healthy bitches. $\mathbf{J}$ Vet Cardiol 2010;12:123-8. 
3. Blanco PG, Tórtora M, Rodríguez R, Arias D, Gobello C. Ultrasonographic assessment of maternal cardiac function and peripheral circulation during normal gestation in dogs. Vet $\mathrm{J}$ 2010; doi:10.1016/j.tvj1.2010.08.013.

4. Gowda RM, Khan IA, Mehta NJ, Vasavada BC, Sacchi TJ. Cardiac arrhythmias in pregnancy: clinical and therapeutic considerations. Int J Cardiol 2003;88:129-33.

5. Baumert M, Seeck A, Faber R, Nalivaiko E, Voss A. Longitudinal changes in QT interval variability and rate adaptation in pregnancies with normal and abnormal uterine perfusion. Hypertens Res 2010;33:555-60.

6. Eghbali M, Wang Y, Toro L, Stefani E. Heart hypertrophy during pregnancy: a better functioning heart? Trends Cardiovasc Med 2006;16:285-91.

7. Eghbali M, Deva R, Alioua A, Minosyan TY, Ruan H, Wang Y, Toro L, Stefani E. Molecular and Functional Signature of Heart Hypertrophy During Pregnancy. Circ Res 2005;96:1208-16.

8. Olsson K, Lagerstedt AS, Bergström A, Häggström J. Change of diurnal heart rate patterns during pregnancy and lactation in dogs (Canis familiaris). Acta Vet Scand 2003;44:105-10. 9. Lúcio CF, Silva LC, Rodrigues JA, Veiga GA, Vannucchi CI. Peripartum haemodynamic status of bitches with normal birth or dystocia. Reprod Domest Anim 2009;44:133-6.

10. Root Kustritz MV. Pregnancy diagnosis and abnormalities of pregnancy in the dog. Theriogenology 2005;64:755-65.

11. Flood JA, Hoover JP. Improvement in myocardial dysfunction in a hypothyroid dog. Can Vet J 2009;50:828-34.

12. Davidson AP. Approaches to Reducing Neonatal Mortality in Dogs. En: Concannon PW, England G, Verstegen J, Linde-Forsberg C, editores. Recent Advances in Small Animal Reproduction. Ithaca, New York, IVIS, 2003; www.ivis.org. Document number A1226.0303. 
13. Münnich A. The pathological newborn in small animals: the neonate is not a small adult. Vet Res Commun 2008;32:S81-5.

14. Ferasin L, Ferasin H, Little CJ. Lack of correlation between canine heart rate and body size in veterinary clinical practice. J Small Anim Pract 2010;51:412-8.

15. Kittleson MD. Electrocardiography. En: Kittelson MD, Kienle RD, editores. Small animal cardiovascular medicine textbook. St. Louis, Mosby; 1998. p. 72-94.

16. Tilley LP. The approach to the electrocardiogram. En: Tilley LP, editor. Essentials of canine and feline electrocardiography. Media, Lippincott Williams \& Wilkins;1992. p. 40-50. 17. Tattersall ML, Dymond M, Hammond T, Valentin JP. Correction of QT values to allow for increases in heart rate in conscious Beagle dogs in toxicology assessment. J Pharmacol Toxicol Methods 2006;53:11-9.

18. Wong AY, Kulandavelu S, Whiteley KJ, Qu D, Langille BL, Adamson SL. Maternal cardiovascular changes during pregnancy and postpartum in mice. Am J Physiol Heart Circ Physiol 2002;282:H918-25.

19. Kienle RD. The effects of systemic disease on the cardiovascular system. En: Kittelson MD, Kienle RD, editores. Small animal cardiovascular medicine textbook. St. Louis, Mosby; 1998. p. 552-560.

20. Kittleson MD. Diagnosis and treatment of arrhythmias. En: Kittelson MD, Kienle RD, editores. Small animal cardiovascular medicine textbook. St. Louis, Mosby; 1998. p. 449-494. 21. Ghaffari MS, Khorami N, Soroori S. Clinical and electrocardiogram findings in a bitch with iatrogenic hypermagnesaemia and hypercalcaemia. Vet Rec 2009;164:176-7.

22. Ozmen N, Cebeci BS, Yiginer O, Muhcu M, Kardesoglu E, Dincturk M. P-wave dispersion is increased in pregnancy due to shortening of minimum duration of P: does this have clinical significance? J Int Med Res 2006;34:468-74. 
23. Bhakta P, Biswas BK, Banerjee B. Peripartum cardiomyopathy: review of the literature. Yonsei Med J 2007;31:731-47.

24. Spier AW, Meurs KM, Muir WW, Lehmkuhl LB, Hamlin RL. Correlation of QT dispersion with indices used to evaluate the severity of familial ventricular arrhythmias in Boxers. Am J Vet Res 2001;62:1481-5.

25. Kron J, Conti JB. Arrhythmias in the pregnant patient: current concepts in evaluation and management. J Interv Card Electrophysiol 2007;19:95-107. 


\section{CONCLUSIONES FINALES}

La función cardíaca y la circulación periférica maternas se modifican a lo largo de la preñez canina. El descenso en la poscarga, el incremento en el gasto cardíaco y la hipertrofia cardíaca resultan de los cambios hemodinámicos que ocurren durante la gestación. Por lo tanto, la adaptación cardíaca materna en las perras juega un papel vital en el mantenimiento de la perfusión uterina óptima para el desarrollo fetal.

En nuestro modelo experimental de gestación anormal canina, algunos parámetros cardiovasculares difirieron de la normalidad antes de que la preñez se interrumpa, permitiendo predecir anomalías gestacionales.

El flujo sanguíneo de las arterias uterina, umbilical y renal fetal difiere entre la gestación canina normal y anormal, permitiendo predecir un desenlace gestacional adverso. Las anormalidades en el trazado Doppler pueden también aparecer antes que los cambios en la frecuencia cardíaca fetal.

En perros, los signos de adaptación cardiovascular materna se alteran durante la gestación con complicaciones obstétricas y preceden a la aparición clínica de enfermedad materna y fetal. Las disimilitudes cardiovasculares en las perras preñadas podrían ser utilizadas para identificar en forma confiable los casos patológicos. Así, la evaluación ecocardiográfica y Doppler de la morfología y función cardíacas podría constituir un método no invasivo válido que contribuya al monitoreo de la salud materna y fetal. 
Durante la preñez canina, algunos parámetros electrocardiográficos comienzan a modificarse desde el día 40 y las preñeces patológicas empiezan a diferir electrocardiográficamente de la normalidad desde el día 30. Por lo tanto, el monitoreo del electrocardiograma materno durante la gestación podría ser un pilar importante en la toma de decisiones médicas en obstetricia canina.

Este Trabajo de tesis aporta nuevos indicadores de bienestar fetal que demuestran presentarse mucho más tempranamente que los escasos indicadores hasta hoy descriptos. Esto resulta vital en la elección de medidas terapéuticas adecuadas para prevenir complicaciones obstétricas que devengan en la pérdida total o parcial de la camada. Los nuevos parámetros que han demostrado tener esta utilidad podrían en el futuro ser objeto de estudio en otras especies domésticas, como la felina, o en otros estadíos reproductivos como el puerperio. Además, los cambios cardiovasculares encontrados han dado origen a nuevas hipótesis sobre la respuesta cardiocirculatoria ante estados de sobrecarga fisiológica, que podrían compartir aspectos o bien diferir con la respuesta a la sobrecarga patológica propia de las enfermedades cardíacas caninas. 